\title{
Novel Aminonaphthoquinone Mannich Bases Derived from Lawsone and their Copper(II) Complexes: Synthesis, Characterization and Antibacterial Activity
}

\author{
Amanda P. Neves, ${ }^{a}$ Cláudia C. Barbosa, ${ }^{a}$ Sandro J. Greco, ${ }^{a, \#}$ Maria D. Vargas, ${ }^{a, *}$ Lorenzo C. Visentin, ${ }^{b}$ \\ Carlos B. Pinheiro, ${ }^{c}$ Antônio S. Mangrich, ${ }^{d}$ Jussara P. Barbosa ${ }^{e}$ and Gisela L. da Costa ${ }^{e}$
}

IIInstituto de Química, Universidade Federal Fluminense, Campus do Valonguinho, Centro, 24020-150 Niterói-RJ, Brazil

${ }^{b}$ Instituto de Química, Universidade Federal do Rio de Janeiro, Ilha do Fundão, 21945-970 Rio de Janeiro-RJ, Brazil

${ }^{c}$ Departamento de Física, Universidade Federal de Minas Gerais, Av. Antônio Carlos, 6627, Pampulha, 31270-901 Belo Horizonte-MG, Brazil

${ }^{d}$ Departamento de Química, Centro Politécnico, Universidade Federal do Paraná, 81531-970 Curitiba-PR, Brazil

eInstituto Oswaldo Cruz, CP 926, 21045-900 Rio de Janeiro-RJ, Brazil

\begin{abstract}
Uma série de novas Bases de Mannich (HL1-HL13) derivadas da 2-hidroxi-1,4-naftoquinona (lausona), benzaldeídos substituídos $\left[\mathrm{C}_{6} \mathrm{H}_{2} \mathrm{R}^{1} \mathrm{R}^{2} \mathrm{R}{ }^{3} \mathrm{C}(\mathrm{O}) \mathrm{H}\right]$ e várias aminas primárias $\left(\mathrm{NH}_{2} \mathrm{R}^{4}\right.$, $\mathrm{R}^{4}=n$-butil, benzil, alil, 2-furfuril) e seus complexos de $\mathrm{Cu}^{2+},\left[\mathrm{Cu}(\mathbf{L 1})_{2}\right]-\left[\mathrm{Cu}(\mathbf{L 1 3})_{2}\right]$, foram sintetizados e caracterizados por métodos analíticos e espectroscópicos. As estruturas dos complexos $1\left(\mathrm{R}^{1}=\mathrm{R}^{2}=\mathrm{R}^{3}=\mathrm{H} ; \mathrm{R}^{4}=\mathrm{Bu}\right), \mathbf{2}\left(\mathrm{R}^{1}=\mathrm{R}^{3}=\mathrm{H} ; \mathrm{R}^{2}=\mathrm{NO}_{2} ; \mathrm{R}^{4}=\mathrm{Bu}\right)$ e $7\left(\mathrm{R}^{1}=\mathrm{OH} ; \mathrm{R}^{2}=\mathrm{R}^{3}=\mathrm{H}\right.$; $\mathrm{R}^{4}=\mathrm{Bu}$ ) foram determinadas por estudos de difração de raios-X de monocristal. Todos os compostos cristalizam em grupos espaciais centrossimétricos, com um cobre no centro de inversão. Dois $\mathbf{L}^{-}$coordenam-se através dos átomos de oxigênio do naftalen-2-olato e do nitrogênio da amina secundária, formando anéis quelatos de seis membros ao redor do átomo de cobre em um ambiente trans $-\mathrm{N}_{2} \mathrm{O}_{2}$. A atividade antimicrobial de todos os compostos foi testada em sete diferentes linhagens de bactérias: Bacillus cereus, Bacillus subtilis, Escherichia coli, Enterococcus faecalis, Klebsiella pneumoniae, Pseudomonas aeruginosa e Staphylococcus aureus. Em geral, as bases de Mannich foram mais ativas que os complexos, sendo $\mathbf{H L 1 1}\left(\mathrm{R}^{1}=\mathrm{OH} ; \mathrm{R}^{2}=\mathrm{H} ; \mathrm{R}^{3}=\mathrm{Me} ; \mathrm{R}^{4}=\mathrm{Bn}\right)$ e HL13 $\left(\mathrm{R}^{1}=\mathrm{OH} ; \mathrm{R}^{2}=\mathrm{H} ; \mathrm{R}^{3}=\mathrm{Br} ; \mathrm{R}^{4}=\mathrm{Bn}\right)$ os inibidores mais potentes. O MIC para o composto mais ativo HL11 contra $S$. Coli foi $20 \mu \mathrm{mol} \mathrm{L}^{-1}(8 \mu \mathrm{g} \mathrm{mL}-1)$, melhor que o cloranfenicol $\left(90 \mu \mathrm{mol} \mathrm{L}^{-1}\right)$ e bem abaixo da maioria dos valores descritos para outras naftoquinonas.
\end{abstract}

A series of novel Mannich bases (HL1-HL13) derived from 2-hydroxy-1,4-naphthoquinone (lawsone), substituted benzaldehydes $\left[\mathrm{C}_{6} \mathrm{H}_{2} \mathrm{R}^{1} \mathrm{R}^{2} \mathrm{R}{ }^{3} \mathrm{C}(\mathrm{O}) \mathrm{H}\right]$ and various primary amines $\left(\mathrm{NH}_{2} \mathrm{R}^{4}\right.$, $\mathrm{R}^{4}=n$-butyl, benzyl, allyl, 2-furfuryl), and their $\mathrm{Cu}^{2+}$ complexes, $\left[\mathrm{Cu}(\mathbf{L 1})_{2}\right]-\left[\mathrm{Cu}(\mathbf{L 1 3})_{2}\right]$, have been synthesized and fully characterized by analytical and spectroscopic methods. The structures of complexes $1\left(\mathrm{R}^{1}=\mathrm{R}^{2}=\mathrm{R}^{3}=\mathrm{H} ; \mathrm{R}^{4}=\mathrm{Bu}\right), \mathbf{2}\left(\mathrm{R}^{1}=\mathrm{R}^{3}=\mathrm{H} ; \mathrm{R}^{2}=\mathrm{NO}_{2} ; \mathrm{R}^{4}=\mathrm{Bu}\right)$ and $7\left(\mathrm{R}^{1}=\mathrm{OH}\right.$; $\mathrm{R}^{2}=\mathrm{R}^{3}=\mathrm{H} ; \mathrm{R}^{4}=\mathrm{Bu}$ ) were determined by single crystal $\mathrm{X}$-ray diffraction studies. All complexes crystallize in centrosymmetric space groups, with a copper atom in the inversion centre. Two $\mathbf{L}^{-}$coordinate through the naphthalen-2-olate oxygen and secondary amine- $\mathrm{N}$ atoms, forming sixmembered chelate rings around the copper atom in a trans $-\mathrm{N}_{2} \mathrm{O}_{2}$ environment; spectroscopic data confirm that the other complexes exhibit similar molecular arrangement. The antimicrobial activity of all compounds has been tested on seven different strains of bacteria: Bacillus cereus, Bacillus subtilis, Escherichia coli, Enterococcus faecalis, Klebsiella pneumoniae, Pseudomonas aeruginosa and Staphylococcus aureus. In general, Mannich bases were more active than complexes, HL11 $\left(\mathrm{R}^{1}=\mathrm{OH} ; \mathrm{R}^{2}=\mathrm{H} ; \mathrm{R}^{3}=\mathrm{Me} ; \mathrm{R}^{4}=\mathrm{Bn}\right)$ and $\mathrm{HL13}\left(\mathrm{R}^{1}=\mathrm{OH} ; \mathrm{R}^{2}=\mathrm{H} ; \mathrm{R}^{3}=\mathrm{Br} ; \mathrm{R}^{4}=\mathrm{Bn}\right)$ being the most potent inhibitors. The MIC for the most active compound HL11 against $S$. Coli was $20 \mu \mathrm{mol} \mathrm{L}{ }^{-1}\left(8 \mu \mathrm{g} \mathrm{mL}^{-1}\right)$, better than Chloramphenicol $\left(90 \mu \mathrm{mol} \mathrm{L}^{-1}\right)$ and well below most values reported for other naphthoquinones.

Keywords: aminonaphthoquinones, copper complexes, Mannich bases, crystal structure determination, antibacterial activity

*e-mail: mdvargas@vm.uff.br

\# Present Address: Universidade Federal do Espírito Santo, Centro Uni-

versitário Norte do Espírito Santo, Rua Humberto de Almeida Franklin,

257, Universitário, 29933-480 São Mateus-ES, Brazil 


\section{Introduction}

Natural and synthetic naphthoquinones are known for a wide range of biological activities, ${ }^{1}$ amongst which anti-cancer, ${ }^{2,3}$ tripanocidal, ${ }^{4}$ molluscicidal, ${ }^{5}$ antimalarial, ${ }^{6}$ leishmaniscide, ${ }^{7}$ bacteriostatic and bactericidal. ${ }^{8,9}$ The most accepted mechanism for the antimicrobial activity of naphthoquinones is based on the generation of reactive oxygen species by two successive reduction processes to form radical anion and dianion species that are toxic to bacteria. ${ }^{10,11}$

It has been shown that the incorporation of amino groups or a nitrogen atom into naphthoquinones often results in increased anticancer, ${ }^{12-14}$ molluscicidal ${ }^{15-17}$ and antibacterial activities. ${ }^{10,18-22}$ We therefore evaluated the antimicrobial activity of a novel series of 2-hydroxy-3alkylamine-1,4-naphthoquinones, known as Mannich bases. These compounds were first synthesized over sixty years ago, ${ }^{23}$ and their antimalarial ${ }^{23,24}$ and molluscicidal ${ }^{25}$ activities have been described. In spite of the fact that metal complexation of a number naphthoquinones or naphthoquinone derived compounds has resulted in increased citoxicity, ${ }^{26}$ antimalarial ${ }^{27,28}$ and anticancer ${ }^{29}$ activity, transition metal complexes of Mannich bases derived from 2-hydroxy-1,4-naphthoquinone (lawsone) have not yet been reported. Herein we describe the synthesis of novel Mannich bases HL1-HL13 from lawsone, a number of primary amines and substituted benzaldehydes and of their copper(II) complexes, $\left[\mathrm{Cu}(\mathbf{L})_{2}\right]$ (1-13) (Scheme 1), their characterization by analytical and spectroscopic techniques, and the X-ray diffraction studies of three complexes. Furthermore, we report the results of antibacterial activity screening of all compounds and discuss growth inhibition as a function of structural changes and metal complexation.

\section{Experimental}

\section{Materials and methods}

Reagents and solvents were used without further purification. Microanalyses were performed using a PerkinElmer CHN 2400 micro analyser at the Central Analítica, Instituto de Química, USP, São Paulo, Brazil. Melting points were obtained with a Mel-Temp II, Laboratory Devices-USA apparatus and are uncorrected. IR spectra (KBr pellets) were recorded on a FT-IR Spectrum One (Perkin Elmer) spectrophotometer. ${ }^{1} \mathrm{H}$ and ${ }^{13} \mathrm{C}$ NMR spectra were recorded with a Varian Unit Plus $300 \mathrm{MHz}$ spectrometer in $\mathrm{CDCl}_{3}$ or $\mathrm{d}^{6}-\mathrm{DMSO}$; coupling constants are reported in Hertz $(\mathrm{Hz})$ and chemical shifts in parts per million (ppm) relative to internal standard $\mathrm{Me}_{4} \mathrm{Si}$. The hydrogen signals were attributed thought coupling constant values and ${ }^{1} \mathrm{H} \times{ }^{1} \mathrm{H}-\mathrm{COSY}$ experiments. Electronic spectra were taken on a Diode Array 8452A (Hewlett Packard-HP) spectrophotometer using spectroscopic grade solvents (Tedia Brazil) in $10^{-3}$ and $10^{-4} \mathrm{~mol} \mathrm{~L}^{-1}$ solutions. Electron paramagnetic resonance (EPR) spectra of the solid samples were obtained at liquid nitrogen temperature $(77 \mathrm{~K})$, on a Bruker ESP300E equipment with modulation frequency of $100 \mathrm{kHz}$, operating at $9.5 \mathrm{GHz}$ (X-band).

\section{Synthesis of the Mannich bases HL1-HL13}

Compounds HL1-HL13 (Figure 1) were synthesised according to the general procedure described in the literature ${ }^{24,30}$ with modifications. They were obtained by reacting a suspension of lawsone $(5 \mathrm{mmol}, 0.870 \mathrm{~g})$, in $15 \mathrm{~mL}$ of ethanol, with the respective amine $(5.5 \mathrm{mmol})$. After formation of the lawsonate solution, the aldehyde (6 mmol) is added and the mixture, left stirring at room<smiles>[R]NC(C1=C(O)C(=O)c2ccccc2C1=O)c1cc([R])c([R])cc1[R]</smiles>

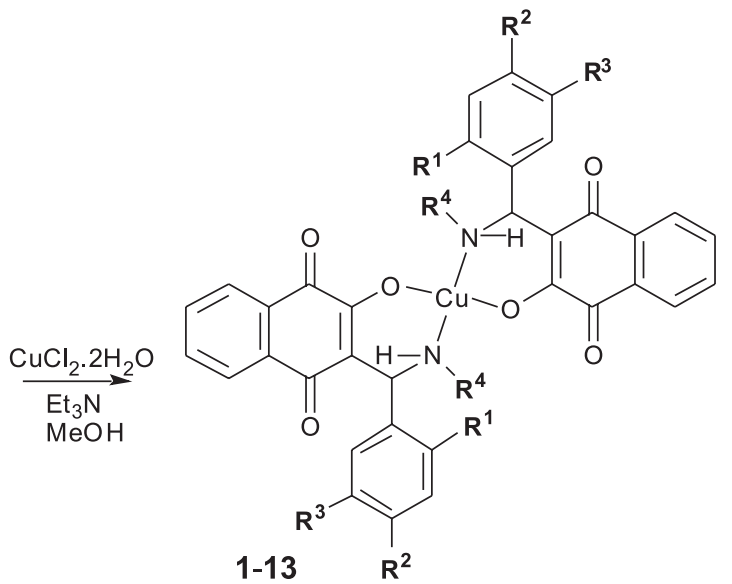

Scheme 1. Synthesis of Mannich bases HL1-HL13 and of complexes 1-13. 


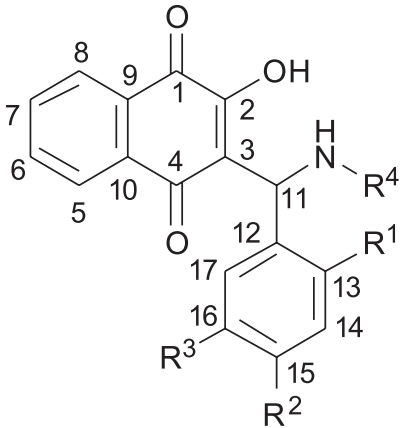

$\mathrm{R}^{1}=\mathrm{R}^{2}=\mathrm{R}^{3}=\mathrm{H} ; \mathrm{R}^{4}=\mathrm{Bu}, \mathrm{HL1}$

$\mathrm{R}^{1}=\mathrm{R}^{3}=\mathrm{H} ; \mathrm{R}^{2}=\mathrm{NO}_{2} ; \mathrm{R}^{4}=\mathrm{Bu}, \mathrm{HL2}$

$\mathrm{R}^{1}=\mathrm{R}^{3}=\mathrm{H} ; \mathrm{R}^{2}=\mathrm{NO}_{2} ; \mathrm{R}^{4}=\mathrm{Bn}, \mathrm{HL} 3$

$\mathrm{R}^{1}=\mathrm{R}^{2}=\mathrm{Cl} ; \mathrm{R}^{3}=\mathrm{H} ; \mathrm{R}^{4}=\mathrm{Bu}, \mathrm{HL} 4$

$\mathrm{R}^{1}=\mathrm{R}^{2}=\mathrm{Cl} ; \mathrm{R}^{3}=\mathrm{H} ; \mathrm{R}^{4}=\mathrm{Bn}, \mathrm{HL} 5$

$\mathrm{R}^{1}=\mathrm{OH} ; \mathrm{R}^{2}=\mathrm{R}^{3}=\mathrm{H} ; \mathrm{R}^{4}=$ alyl, $\mathrm{HL6}$

$\mathrm{R}^{1}=\mathrm{OH} ; \mathrm{R}^{2}=\mathrm{R}^{3}=\mathrm{H} ; \mathrm{R}^{4}=\mathrm{Bu}, \mathrm{HL7}$

Figure 1. Mannich bases HL1-HL13.

temperature for $12 \mathrm{~h}$ in the dark. The orange solids were filtered, washed with ethanol, diethyl eter and dried under vacuum.

3-[N-(n-butyl)aminobenzyl]-2-hydroxy-1,4naphthoquinone (HL1)

From $0.54 \mathrm{~mL}$ of butylamine and $0.61 \mathrm{~mL}$ of benzaldehyde. Yield: $1.258 \mathrm{~g}, 74 \%$, mp $143-144{ }^{\circ} \mathrm{C}$. Anal. Calc. for $\mathrm{C}_{21} \mathrm{H}_{21} \mathrm{NO}_{3}$ : C, 75.20; H, 6.31; N, 4.18\%. Found: C, 75.14; H, 6.29; N, 4.23\%. IR (KBr) $v_{\max } / \mathrm{cm}^{-1}$ : 3435 (O-H), 3060 (C-H), 2960 (C-H), 2933 (C-H), 1680 $(\mathrm{C}=\mathrm{O}), 1588(\mathrm{C}=\mathrm{C}), 1529(\delta \mathrm{N}-\mathrm{H}), 1276(\mathrm{C}-\mathrm{O}) .{ }^{1} \mathrm{H}$ NMR (DMSO-d ${ }^{6}, 300 \mathrm{MHz}$ ): $\delta$ (ppm) 8.02 (ddd, $J$ 7.60, 1.22, $0.45 \mathrm{~Hz}, 1 \mathrm{H}, H 5$ or $H 8$ ); 7.93 (ddd, $J 7.57,1.41,0.45 \mathrm{~Hz}$, $1 \mathrm{H}, H 8$ or $H 5) ; 7.81$ (td, $J 7.60,7.60,1.41 \mathrm{~Hz}, 1 \mathrm{H}, H 6$ or $H 7) ; 7.73-7.66$ (m, 3H, H7 or $H 6$ and $P h) ; 7.49-7.38$ (m, $3 \mathrm{H}, P h) ; 5.59$ (br s, 1H, H11); 2.97 (br t, J 7.65, $7.65 \mathrm{~Hz}$, 2H, H19); 1.76-1.64 (m, 2H, H2O); 1.48-1.34 (m, 2H, H2I); 0.94 (t, J 7.36, 7.36 Hz, 3H, H22). ${ }^{13} \mathrm{C}$ NMR (DMSO-d ${ }^{6}, 75$ MHz): $\delta$ (ppm) 184.6, 178.7, 170.8, 138.9, 134.8, 134.0, 131.7, 131.1, 128.6, 128.1, 127.9, 125.6, 125.3, 111.5, 59.0, 45.6, 27.9, 19.5, 13.7. UV-Vis $\left(\mathrm{CHCl}_{3}\right) \lambda / \mathrm{nm}, \log \varepsilon: 267$ (4.19), 338 (3.15), 437(3.20).

3-[N-(n-butyl)amino-3-nitrobenzyl]-2-hydroxy-1,4naphthoquinone (HL2)

From $0.54 \mathrm{~mL}$ of butylamine and $0.907 \mathrm{~g}$ of $p$-nitrobenzaldehyde. Yield: $1.141 \mathrm{~g}, 60 \%, \mathrm{mp} 172^{\circ} \mathrm{C}$. Anal. Calc. for $\mathrm{C}_{21} \mathrm{H}_{20} \mathrm{~N}_{2} \mathrm{O}_{5}: \mathrm{C}, 66.31 ; \mathrm{H}, 5.30 ; \mathrm{N}, 7.36 \%$. Found: $\mathrm{C}$, 66.20; H, 5.49; N, 7.51\%. IR (KBr) $v_{\max } / \mathrm{cm}^{-1}: 3437(\mathrm{O}-\mathrm{H})$, $3086(\mathrm{C}-\mathrm{H}), 2962(\mathrm{C}-\mathrm{H}), 1683(\mathrm{C}=\mathrm{O}), 1588(\mathrm{C}=\mathrm{C}), 1520$

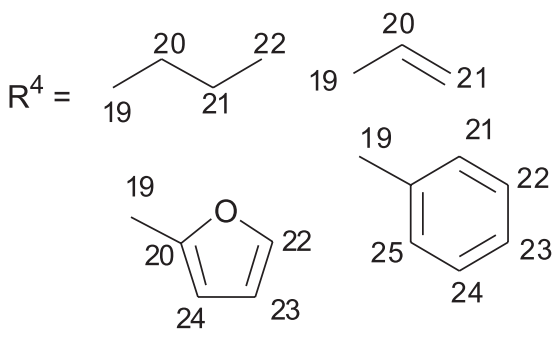

$\mathrm{R}^{1}=\mathrm{OH} ; \mathrm{R}^{2}=\mathrm{R}^{3}=\mathrm{H} ; \mathrm{R}^{4}=\mathrm{Bn}, \mathrm{HL} 8$

$\mathrm{R}^{1}=\mathrm{OH} ; \mathrm{R}^{2}=\mathrm{R}^{3}=\mathrm{H} ; \mathrm{R}^{4}=\mathrm{CH}_{2} \mathrm{C}_{4} \mathrm{H}_{3} \mathrm{O}, \mathrm{HL} \mathbf{9}$

$\mathrm{R}^{1}=\mathrm{OH} ; \mathrm{R}^{2}=\mathrm{H} ; \mathrm{R}^{3}=\mathrm{Me} ; \mathrm{R}^{4}=\mathrm{Bu}, \mathrm{HL} 10$

$\mathrm{R}^{1}=\mathrm{OH} ; \mathrm{R}^{2}=\mathrm{H} ; \mathrm{R}^{3}=\mathrm{Me} ; \mathrm{R}^{4}=\mathrm{Bn}, \mathrm{HL11}$

$\mathrm{R}^{1}=\mathrm{OH} ; \mathrm{R}^{2}=\mathrm{H} ; \mathrm{R}^{3}=\mathrm{Br} ; \mathrm{R}^{4}=\mathrm{Bu}, \mathrm{HL12}$

$\mathrm{R}^{1}=\mathrm{OH} ; \mathrm{R}^{2}=\mathrm{H} ; \mathrm{R}^{3}=\mathrm{Br} ; \mathrm{R}^{4}=\mathrm{Bn}, \mathrm{HL} 13$ $(\delta \mathrm{N}-\mathrm{H}), 1281(\mathrm{C}-\mathrm{O}) .{ }^{1} \mathrm{H}$ NMR (DMSO-d $\left.{ }^{6}, 300 \mathrm{MHz}\right)$ : $\delta$ (ppm) 8.33 (d, J $8.85 \mathrm{~Hz}, 2 \mathrm{H}, H 14 ;$ H16); 8.03 (ddd, $J$ 7.62, 1.34, $0.49 \mathrm{~Hz}, 1 \mathrm{H}, H 5$ or $H 8) ; 7.97$ (d, $J 8.85 \mathrm{~Hz}$, 2H, H13; H17): 7.93 (ddd, J 7.62, 1.39, $0.49 \mathrm{~Hz}, 1 \mathrm{H}, H 8$ or $H 5) ; 7.82$ (td, $J 7.62,7.62,1.39 \mathrm{~Hz}, 1 \mathrm{H}, H 6$ or $H 7$ ); $7.70(\mathrm{td}, J 7.62,7.62,1.34 \mathrm{~Hz}, 1 \mathrm{H}, H 7$ or $H 6) ; 5.78(\mathrm{~s}, 1 \mathrm{H}$, $H 11$ ); 3.02 (t, $J 7.67,7.67 \mathrm{~Hz}, 2 \mathrm{H}, H 19) ; 1.73$ (t, $J 7.67$, $7.67 \mathrm{~Hz}, 2 \mathrm{H}, H 20) ; 1.40$ (sx, J 7.33 Hz, 2H, H21); 0.95 (t, $J$ 7.33, 7.33 Hz, 3H, H22). ${ }^{13} \mathrm{C}$ NMR (DMSO-d ${ }^{6}, 75 \mathrm{MHz}$ ): $\delta(\mathrm{ppm}) 184.2,178.5,170.7,146.8,146.0,134.6,133.9$, 131.6, 131.1, 128.6, 125.5, 123.5, 110.3, 57.9, 45.6, 27.8, 19.4, 13.6. UV-Vis $\left(\mathrm{CHCl}_{3}\right) \lambda / \mathrm{nm}, \log \varepsilon: 268$ (4.36), 336 (3.60), 427(3.26).

3-[N-(benzyl)amino-3-nitrobenzyl]-2-hydroxy-1,4naphthoquinone (HL 3)

From $0.60 \mathrm{~mL}$ of benzyllamine and $0.907 \mathrm{~g}$ of $p$-nitrobenzaldehyde. Yield: $(1.513 \mathrm{~g}, 73 \%)$; mp $139-140{ }^{\circ} \mathrm{C}$. Anal. Calc. for $\mathrm{C}_{24} \mathrm{H}_{18} \mathrm{~N}_{2} \mathrm{O}_{5}: \mathrm{C}, 69.56 ; \mathrm{H}, 4.38 ; \mathrm{N}, 6.76 \%$. Found: C, 68.80; H, 4.39; N, 6.67\%. IR (KBr) $v_{\max } / \mathrm{cm}^{-1}$ : $3445(\mathrm{O}-\mathrm{H}), 3066$ (C-H), 3032 (C-H), 2969 (C-H), 1677 $(\mathrm{C}=\mathrm{O}), 1592(\mathrm{C}=\mathrm{C}), 1523(\delta \mathrm{N}-\mathrm{H}), 1272(\mathrm{C}-\mathrm{O}) .{ }^{1} \mathrm{H}$ NMR (DMSO-d 6 , $300 \mathrm{MHz}): \delta(\mathrm{ppm}) 8.31(\mathrm{~d}, J 8.86 \mathrm{~Hz}, 2 \mathrm{H}$, H14; H16); 8.04 (ddd, $J 7.60,1.38,0.40 \mathrm{~Hz}, 1 \mathrm{H}, H 5$ or $H 8$ ); 7.95 (ddd, J 7.48, $1.41,0.40 \mathrm{~Hz}, 1 \mathrm{H}, H 8$ or $H 5$ ); 7.92 (d, J $8.86 \mathrm{~Hz}, 2 \mathrm{H}, H 13 ; H 17) ; 7.83$ (td, J 7.60, 7.60, 1.41 $\mathrm{Hz}, 1 \mathrm{H}, H 6$ or $H 7) ; 7.70$ (td, J 7.48, 7.48, $1.38 \mathrm{~Hz}, 1 \mathrm{H}, H 7$ or H6); 7.52-7.49 (m, 5H, Ph); 5.76 (s, 1H, H11); 4.28 (s, $2 \mathrm{H}$, H19). ${ }^{13} \mathrm{C}$ NMR (DMSO-d6, $75 \mathrm{MHz}$ ): $\delta$ (ppm) 184.1, 178.6, 170.7, 146.9, 145.7, 134.6, 133.8, 132.1, 131.6, $131.1,130.2,128.9,128.8,128.6,125.5,125.2,123.5$, 
110.1, 57.8, 49.3. UV-Vis $\left(\mathrm{CH}_{2} \mathrm{Cl}_{2}\right) \lambda / \mathrm{nm}, \log \varepsilon: 273$ (4.47), 337 (3.74), 389 (3.22).

3-[N-(n-butyl)amino-2,4-diclorobenzyl]-2-hydroxy-1,4naphthoquinone (HL4)

From $0.60 \mathrm{~mL}$ of benzylamine and $1.050 \mathrm{~g}$ of 2-4-dicloro-benzaldehyde. Yield: $1.071 \mathrm{~g}, 53 \%$; mp 142$143{ }^{\circ} \mathrm{C}$. Anal. Calc. for $\mathrm{C}_{21} \mathrm{H}_{19} \mathrm{Cl}_{2} \mathrm{NO}_{3}: \mathrm{C}, 62.39 ; \mathrm{H}, 4.74$; $\mathrm{N}, 3.46 \%$. Found: C, $62.00 ; \mathrm{H}, 4.64 ; \mathrm{N}, 3.51 \%$. IR (KBr) $v_{\text {max }} / \mathrm{cm}^{-1}: 3437(\mathrm{O}-\mathrm{H}), 3064(\mathrm{C}-\mathrm{H}), 2958(\mathrm{C}-\mathrm{H}), 2870(\mathrm{C}-$ $\mathrm{H}), 1678(\mathrm{C}=\mathrm{O}), 1616(\mathrm{C}=\mathrm{C}), 1588(\mathrm{C}=\mathrm{C}), 1531(\delta \mathrm{N}-\mathrm{H})$, 1272 (C-O). ${ }^{1} \mathrm{H}$ NMR (DMSO-d ${ }^{6}, 300 \mathrm{MHz}$ ): $\delta$ (ppm) 8.01 (dd, $J 7.46,1.33 \mathrm{~Hz}, 1 \mathrm{H}, H 5$ or $H 8) ; 7.97$ (dd, $J$ 7.46, 1.37 $\mathrm{Hz}, 1 \mathrm{H}, H 8$ or $H 5) ; 7.96(\mathrm{~d}, J 8.51 \mathrm{~Hz}, 1 \mathrm{H}, H 17) ; 7.83$ (td, $J$ 7.46, 7.46, $1.37 \mathrm{~Hz}, 1 \mathrm{H}, H 6$ or $H 7) ; 7.76(\mathrm{~d}, J 2.19,1 \mathrm{H}$, H14); 7.72 (td, J 7.46, 7.46, $1.33 \mathrm{~Hz}, 1 \mathrm{H}, H 7$ or $H 6) ; 7.55$ (dd, J 8.51, 2.19, 1H, H16); 5.99 (s, 1H, H11); 3.05 (br t, $J$ J.35, 7.35 Hz, 2H, H19); 1.80-1.65 (m, 2H, H2O); 1.381.34 (m, 2H, H2l); 0.96 (t, J 7.28, 7.28 Hz, 3H, H22). ${ }^{13} \mathrm{C}$ NMR (DMSO-d $\left.{ }^{6}, 75 \mathrm{MHz}\right): \delta$ (ppm) 184.1, 178.9, 171.3, 134.8, 134.5, 134.4, 133.9, 133.8, 131.9, 131.6, 131.1, 128.8, 127.7, 125.5, 125.2, 109.6, 55.4, 45.9, 27.7, 19.3, 13.5. UV-Vis $\left(\mathrm{CHCl}_{3}\right) \lambda / \mathrm{nm}, \log \varepsilon: 265$ (4.29), 337 (3.42), 422 (3.29).

3-[N-(n-benzyl)amino-2,4-diclorobenzyl]-2-hydroxy-1,4naphthoquinone (HL5)

From $0.54 \mathrm{~mL}$ of butylamine and $1.050 \mathrm{~g}$ of 2-4-diclorobenzaldehyde. Yield: $1.731 \mathrm{~g}, 79 \%$; mp $145-146{ }^{\circ} \mathrm{C}$. Anal. Calc. for $\mathrm{C}_{24} \mathrm{H}_{17} \mathrm{Cl}_{2} \mathrm{NO}_{3}$ : C, 65.77; $\mathrm{H}, 3.91 ; \mathrm{N}, 3.20 \%$. Found: C, 65.67; H, 3.87; N, 3.24\%. IR (KBr) $v_{\max } / \mathrm{cm}^{-1}$ : 3453 (O-H), 3065 (C-H), 3033 (C-H), 2969 (C-H), 1677 $(\mathrm{C}=\mathrm{O}), 1592(\mathrm{C}=\mathrm{C}), 1523(\delta \mathrm{N}-\mathrm{H}), 1271(\mathrm{C}-\mathrm{O}) .{ }^{1} \mathrm{H}$ NMR (DMSO-d $\left.{ }^{6}, 300 \mathrm{MHz}\right): \delta$ (ppm) $8.02(\mathrm{ddd}, J$ 7.37, 1.50, $0.47 \mathrm{~Hz}, 1 \mathrm{H}, H 5$ or $H 8) ; 8.00$ (dd, J 7.37, $1.50,0.47 \mathrm{~Hz}$, 1H, $H 8$ or $H 5) ; 7.86$ (d, $J 8.63 \mathrm{~Hz}, 1 \mathrm{H}, H 17) ; 7.84$ (td, $J$ 7.46, 7.46, $1.50 \mathrm{~Hz}, 1 \mathrm{H}, H 6$ or H7); $7.74(\mathrm{td}, J$ 7.46, 7.46, $1.50 \mathrm{~Hz}, 1 \mathrm{H}, H 7$ or $H 6) ; 7.73(\mathrm{~d}, J 2.16 \mathrm{~Hz}, 1 \mathrm{H}, H 14)$; 7.55-7.49 (m, 6H, H16; Ph); 5.78 (s, 1H, H1 ); 4.35 (d, J $13.00 \mathrm{~Hz}, 1 \mathrm{H}, H 19) ; 4.21$ (d, $\left.J 13.00 \mathrm{~Hz}, 1 \mathrm{H}, H 19^{\prime}\right) .{ }^{13} \mathrm{C}$ NMR (DMSO-d $\left.{ }^{6}, 75 \mathrm{MHz}\right): \delta$ (ppm) 183.9, 179.3, 171.3, 134.6, 134.5, 134.4, 134.0, 133.9, 132.1, 132.0, 131.7, $131.2,130.3,128.9,128.8,128.6,127.8,125.6,125.2$, 109.4, 55.1, 49.7. UV-Vis $\left(\mathrm{CHCl}_{3}\right) \lambda / \mathrm{nm}, \log \varepsilon: 275$ (4.14), 334 (3.40), 400 (3.10).

3-[N-(alyl)amino-2-hydroxybenzyl]-2-hydroxy-1,4naphthoquinone (HL6)

From $0.41 \mathrm{~mL}$ of alylamine and $0.63 \mathrm{~mL}$ of 2-hydroxybenzaldehyde. Yield: $1.475 \mathrm{~g}, 88 \%$; mp $164-165^{\circ} \mathrm{C}$. Anal. Calc. for $\mathrm{C}_{20} \mathrm{H}_{17} \mathrm{NO}_{4}$ : C, 71.63; H, 5.11; N, 4.18\%.
Found: C, 71.50; H, 5.05; N, 4.27\%. IR (KBr) $v_{\max } / \mathrm{cm}^{-1}$ : $3255(\mathrm{O}-\mathrm{H}), 3072(\mathrm{C}-\mathrm{H}), 2980(\mathrm{C}-\mathrm{H}), 2950(\mathrm{C}-\mathrm{H}), 1678$ $(\mathrm{C}=\mathrm{O}), 1593(\mathrm{C}=\mathrm{C}), 1561(\delta \mathrm{N}-\mathrm{H}), 1277(\mathrm{C}-\mathrm{O}) .{ }^{1} \mathrm{H}$ NMR (DMSO-d $\left.{ }^{6}, 300 \mathrm{MHz}\right): \delta$ (ppm) 8.02 (ddd, $J$ 7.61, 1.35, $0.47 \mathrm{~Hz}, 1 \mathrm{H}, H 5$ or $H 8) ; 7.97$ (dd, $J 7.53,1.41,0.47 \mathrm{~Hz}, 1 \mathrm{H}$, $H 8$ or $H 5) ; 7.83$ (td, $J 7.47,7.47,1.41 \mathrm{~Hz}, 1 \mathrm{H}, H 6$ or $H 7)$; $7.73(\mathrm{td}, J 7.47,7.47,1.35 \mathrm{~Hz}, 1 \mathrm{H}, H 7$ or $H 6) ; 7.42$ (dd, $J$ $7.69,1.65 \mathrm{~Hz}, 1 \mathrm{H}, H 14$ or H17); $7.26(\mathrm{td}, J 8.06,8.06,1.65$ $\mathrm{Hz}, 1 \mathrm{H}, H 16$ or H15); 6.96 (dd, J 8.06, $0.99 \mathrm{~Hz}, 1 \mathrm{H}, H 17$ or H14); 6.86 (td, J 7.69, 7.69, $0.99 \mathrm{~Hz}, 1 \mathrm{H}, H 15$ or H16); 6.10-5.95 (m, 2H, H20); 5.85 (s, H11); 5.50-5.42 (m, 2H, H21); 3.74-3.60 (m, 2H, H19). ${ }^{13} \mathrm{C}$ NMR (DMSO-d ${ }^{6}, 75$ MHz): $\delta$ (ppm) 184.1, 179.6, 171.5, 155.5, 134.6, 133.9, 131.6, 131.1, 129.8, 129.4, 128.6, 125.5, 125.2, 123.8, 122.0, 119.1, 116.1, 110.1, 53.3, 47.9. UV-Vis (DMSO) $\lambda / \mathrm{nm}, \log \varepsilon: 278$ (4.34), 452 (3.33).

3-[N-(n-butyl)amino-2-hydroxybenzyl]-2-hydroxy-1,4naphthoquinone (HL7)

From $0.54 \mathrm{~mL}$ of butylamine and $0.63 \mathrm{~mL}$ of 2-hydroxybenzaldehyde. Yield: $1.405 \mathrm{~g}, 80 \%$; mp $137-138^{\circ} \mathrm{C}$ (with dec.). Anal. Calc. for $\mathrm{C}_{21} \mathrm{H}_{21} \mathrm{NO}_{4}: \mathrm{C}, 71.78 ; \mathrm{H}, 6.02 ; \mathrm{N}$, 3.99\%. Found: C, 71.12; H, 6.03; N, 3.92\%. IR (KBr) $v_{\max }$ ' $\mathrm{cm}^{-1}: 3233(\mathrm{O}-\mathrm{H}), 3069(\mathrm{C}-\mathrm{H}), 2959(\mathrm{C}-\mathrm{H}), 2875(\mathrm{C}-\mathrm{H})$, $1681(\mathrm{C}=\mathrm{O}), 1590(\mathrm{C}=\mathrm{C}), 1528(\delta \mathrm{N}-\mathrm{H}), 1275(\mathrm{C}-\mathrm{O}) .{ }^{1} \mathrm{H}$ NMR (DMSO-d ${ }^{6}, 300 \mathrm{MHz}$ ): $\delta$ (ppm) 8.02 (ddd, $J$ 7.66, $1.35,0.50 \mathrm{~Hz}, 1 \mathrm{H}, H 5$ or $H 8$ ); 7.98 (ddd, $J 7.47 ; 1.35 ; 0.50$ $\mathrm{Hz}, 1 \mathrm{H}, H 8$ or $H 5) ; 7.84$ (td, $J 7.47,7.47,1.35 \mathrm{~Hz}, 1 \mathrm{H}, H 6$ or $H 7) ; 7.73(\mathrm{td}, J 7.47,7.47,1,35 \mathrm{~Hz}, 1 \mathrm{H}, H 7$ or $H 8) ; 7.44$ (dd, $J 7.74,1.56 \mathrm{~Hz}, 1 \mathrm{H}, H 14$ or H17); 7.27 (td, $J 8.03$, $8.03,1.56 \mathrm{~Hz}, 1 \mathrm{H}, H 16$ or H15); 6.99 (dd, J 8.03, $1.05 \mathrm{~Hz}$, $1 \mathrm{H}, H 17$ or H14); 6.86 (td, J 7.74, 7.74, $1.05 \mathrm{~Hz}, 1 \mathrm{H}, H 15$ or H16); 5.86 (s, 1H, H11); 3.03 (t, J 7.50, $7.50 \mathrm{~Hz}, 2 \mathrm{H}$, H19); 1.79-1.66 (m, 2H, H2O); 1.50-1.38 (m, 2H, H21); 0.96 (br t, $J 7.33,7.33 \mathrm{~Hz}, 3 \mathrm{H}, H 22$ ). ${ }^{13} \mathrm{C}$ NMR (DMSO-d ${ }^{6}$, $75 \mathrm{MHz}$ ): $\delta$ (ppm) 184.1, 179.6, 171.6, 155.4, 134.5, 133.9, 131.6, 131.2, 129.6, 128.7, 125.5, 125.2, 123.6, 119.1, 115.9, 109.9, 54.1, 45.7, 27.8, 19.4, 13.5. UV-Vis (DMSO) $\lambda / \mathrm{nm}, \log \varepsilon: 277$ (4.33), 450 (3.30).

3-[N-(benzyl)amino-2-hydroxybenzyl]-2-hydroxy-1,4naphthoquinone (HL8)

From $0.60 \mathrm{~mL}$ of benzylamine and $0.63 \mathrm{~mL}$ of 2-hydroxy-benzaldehyde. Yield: 1.792 g, 93\%; mp 165$166^{\circ} \mathrm{C}$. Anal. Calc. for $\mathrm{C}_{24} \mathrm{H}_{19} \mathrm{NO}_{4} \mathrm{C}, 74.79 ; \mathrm{H}, 4.97 ; \mathrm{N}$, $3.63 \%$. Found: C, 74.78; H, 4.90; N, 3.72\%. IR (KBr) $v_{\max } / \mathrm{cm}^{-1}: 3436(\mathrm{O}-\mathrm{H}), 3067(\mathrm{C}-\mathrm{H}), 2744(\mathrm{C}-\mathrm{H}), 1681$ $(\mathrm{C}=\mathrm{O}), 1589(\mathrm{C}=\mathrm{C}), 1516(\delta \mathrm{N}-\mathrm{H}), 1276(\mathrm{C}-\mathrm{O}) .{ }^{1} \mathrm{H}$ NMR (DMSO-d ${ }^{6}, 300 \mathrm{MHz}$ ): $\delta$ (ppm) 8.04 (ddd, $J$ 7.50, 1.36, $0.48 \mathrm{~Hz}, 1 \mathrm{H}, H 5$ or $H 8$ ); 7.99 (ddd, $J 7.50,1.43,0.48 \mathrm{~Hz}$, 1H, H8 or H5); 7.84 (td, J 7.50, 7.50, $1.43 \mathrm{~Hz}, 1 \mathrm{H}, H 6$ 
or $H 7) ; 7.73(\mathrm{td}, J 7.50,7.50,1.36 \mathrm{~Hz}, 1 \mathrm{H}, H 7$ or $H 6)$; 7.51-7.46 (m, 5H,Ph); $7.38(\mathrm{dd}, J 7.71,1.62 \mathrm{~Hz}, 1 \mathrm{H}$, $H 14$ or $H 17) ; 7.24(\mathrm{td}, J 8.05 ; 8.05,1.62 \mathrm{~Hz}, 1 \mathrm{H}, H 16$ or H15); 6.94 (dd, J 8.05, $1.00 \mathrm{~Hz}, 1 \mathrm{H}, H 17$ or H14); $6.84(\mathrm{td}, J 7.71,7.71,1.00 \mathrm{~Hz}, 1 \mathrm{H}, H 15$ or H16); 5.82 (s, $1 \mathrm{H}, H 11) ; 4.26(\mathrm{~d}, J 13.09,1 \mathrm{H}) ; 4.17(\mathrm{~d}, J 13.09,1 \mathrm{H})$. ${ }^{13} \mathrm{C}$ NMR (DMSO-d 6 , $75 \mathrm{MHz}$ ): $\delta$ (ppm) 184.1, 179.7, $171.5,155.6,134.6,133.9,132.8,131.7,131.1,130.0$, $129.4,128.9,128.7,128.5,125.5,125.2$, 123.6, 119.0, 115.9, 110.0, 53.8, 49.4. UV-Vis (DMSO) $\lambda / \mathrm{nm}, \log \varepsilon$ : 277 (4.29), 443 (3.25).

3-[N-(furfurylmethyl)amino-2-hydroxybenzyl]-2-hydroxy1,4-naphthoquinone (HL9)

From furfurylamine $(0.49 \mathrm{~mL})$ and 2-hydroxybenzaldehyde (0.63 mL). Yield: $1.653 \mathrm{~g}, 88 \%$; mp $138^{\circ} \mathrm{C}$. Anal. Calc. for $\mathrm{C}_{24} \mathrm{H}_{19} \mathrm{NO}_{4} \cdot \mathrm{H}_{2} \mathrm{O}: \mathrm{C}, 67.17 ; \mathrm{H}, 4.87 ; \mathrm{N}$, $3.56 \%$. Found: C, 68.94; H, 4.99; N, 3.69\%. IR (KBr) $\mathrm{v}_{\max } / \mathrm{cm}^{-1}: 3234(\mathrm{O}-\mathrm{H}), 2943(\mathrm{C}-\mathrm{H}), 1683(\mathrm{C}=\mathrm{O}), 1591$ $(\mathrm{C}=\mathrm{C}), 1551(\delta \mathrm{N}-\mathrm{H}), 1279(\mathrm{C}-\mathrm{O}) .{ }^{1} \mathrm{H}$ NMR (DMSO-d ${ }^{6}$, $300 \mathrm{MHz}): \delta$ (ppm) 8.02 (br d, J 7.61 Hz, 1H, H5 or H8); 7.97 (br d, J $7.56 \mathrm{~Hz}, 1 \mathrm{H}, H 8$ or H5); 7.83 (td, J 7.61, $7.61,1.20 \mathrm{~Hz}, 1 \mathrm{H}, \mathrm{H6}$ or H7); 7.72 (td, J 7.56, 7.56, 1.20 $\mathrm{Hz}, 1 \mathrm{H}, H 7$ or H6); 7.51-7.46 (m, 5H, Ph); 7.32 (br d, J $7.89 \mathrm{~Hz}, 1 \mathrm{H}, H 14$ or H17); 7.23 (br td, J 7.89; 7.89, 1.62 $\mathrm{Hz}, 1 \mathrm{H}, H 16$ or H15); 6.92 (br d, J $7.89 \mathrm{~Hz}, 1 \mathrm{H}, H 17$ or H14); 6.82 (br t, J $7.48 \mathrm{~Hz}, 1 \mathrm{H}, H 15$ or H16); 5.78 (s, $1 \mathrm{H}, H 11) ; 4.23$ (d, J 14.51, 1H); 4.17 (d, J 14.51, 1H). ${ }^{13} \mathrm{C}$ NMR (DMSO-d $\left.{ }^{6}, 75 \mathrm{MHz}\right): \delta$ (ppm) 184.2, 179.7, 171.4, 155.7, 143.9, 134.6, 133.9, 131.7, 131.2, 129.4, $128.6,125.5,125.3,123.7,119.2,119.0,116.0,111.6$, 111.0, 110.1, 53.9, 41.9. UV-Vis $\left(\mathrm{CHCl}_{3}\right) \lambda / \mathrm{nm}, \log \varepsilon$ : 272 (4.03), 305 (3.93), 366 (3.74).

3-[N-(n-butyl)amino-2-hydroxy-5-methyl-benzyl]-2hydroxy-1,4-naphthoquinone (HL10)

From butylamine $(0.54 \mathrm{~mL})$ and 2-hydroxy-5-methylbenzaldehyde (0.817 g). Yield: $1.279 \mathrm{~g}, 70 \%$; mp 154$155^{\circ} \mathrm{C}$. Anal. Calc. for $\mathrm{C}_{22} \mathrm{H}_{23} \mathrm{NO}_{4}$ : C, 72.31; H, 6.34; N, 3.83. Found: C, 71.15; H, 6.28; N, 3.90\%. IR (KBr) $v_{\text {max }} /$ $\mathrm{cm}^{-1}$ : 3065, 2959, 2870, 1686, 1591, 1551, 1508, 1477 1432, 1374, 1278. ${ }^{1} \mathrm{H}$ NMR (DMSO-d $\left.{ }^{6}, 300 \mathrm{MHz}\right): \delta(\mathrm{ppm})$ 8.02 (ddd, J 7.67; 1.30; $0.40 \mathrm{~Hz}, 1 \mathrm{H}, H 5$ or H8); 7.98 (ddd, $J 7.54 ; 1.37 ; 0.40 \mathrm{~Hz}, 1 \mathrm{H}, H 8$ or H5): 7.84 (td, J 7.54; 7.54; $1.30 \mathrm{~Hz}, 1 \mathrm{H}, H 6$ or $H 7) ; 7.73$ (td, J 7.47; 7.47; $1.37 \mathrm{~Hz}$, 1H, H7 or H6); 7.25 (d, J $1.94 \mathrm{~Hz}, 1 \mathrm{H}, H 17): 7.07$ (dd, J 8.22, 1.94 Hz, 1H, H15); 6.87 (d, J 8.22 Hz, H14); 5.81 (s, $1 \mathrm{H}, H 11) ; 3.00$ (t, J 7.55, $7.55 \mathrm{~Hz}, 2 \mathrm{H}, H 19) ; 2.25$ (s, 3H, $\mathrm{CH}_{3}$ ); 1.77-1.65 (m, 2H, H2O); 1.48-1.34 (m, 2H, H21); 0.96 (t, J 7.34, $7.34 \mathrm{~Hz}, 3 \mathrm{H}, \mathrm{H} 22) .{ }^{13} \mathrm{C}$ NMR (DMSO-d ${ }^{6}$, $75 \mathrm{MHz}): \delta$ (ppm) 184.1, 179.5, 171.4, 153.1, 134.5, 133.9,
$131.6,131.1,129.8,128.6,127.5,125.5,125.2,123.5$; $115.9 ; 110.2 ; 54.1 ; 45.7 ; 27.9 ; 20.3 ; 19.4 ; 13.5$. UV-Vis $\left(\mathrm{CHCl}_{3}\right) \lambda / \mathrm{nm}, \log \varepsilon: 274$ (4.10), 325 (3.63), 373 (3.38), 470 (2.93).

3-[N-(benzyl)amino-2-hydroxy-5-methyl-benzyl]-2hydroxy-1,4-naphthoquinone (HL11)

From benzylamine $(0.60 \mathrm{~mL})$ and 2-hydroxy-5-methylbenzaldehyde $(0.817 \mathrm{~g})$. Yield: $1.338 \mathrm{~g}, 67 \%$; mp $147-$ $148{ }^{\circ} \mathrm{C}$. Anal. Calc. for $\mathrm{C}_{25} \mathrm{H}_{21} \mathrm{NO}_{4}$ : C, 75.17; H, 5.30; N, 3.51. Found: C, 74.54; H, 5.36; N, 3.51\%. IR (KBr) $v_{\max } /$ $\mathrm{cm}^{-1}: 3266 ; 3065 ; 2957 ; 2862 ; 1686 ; 1590 ; 1539 ; 1274 ; 1221$. ${ }^{1} \mathrm{H}$ NMR (DMSO-d $\left.{ }^{6}, 300 \mathrm{MHz}\right): \delta$ (ppm) 8.04 (ddd, J 7.61, $1.39,0.43 \mathrm{~Hz}, 1 \mathrm{H}, H 5$ or H8); 7.99 (ddd, J 7.54, 1.41, 0.43 $\mathrm{Hz}, 1 \mathrm{H}, H 8$ or H5); 7.84 (td, J 7.43, 7.43, $1.41 \mathrm{~Hz}, 1 \mathrm{H}, H 6$ or H7); 7.73 (td, J 7.43, 7.43, $1.39 \mathrm{~Hz}, 1 \mathrm{H}, H 7$ or H6); 7.50-7.47 (m, 5H, Ph); 7.20 (d, J $2.03 \mathrm{~Hz}, 1 \mathrm{H}, H 17) ; 7.04$ (dd, J 8.16, $2.03 \mathrm{~Hz}, 1 \mathrm{H}, H 15) ; 6.83$ (d, J $8.16 \mathrm{~Hz}, 1 \mathrm{H}, H 14) ; 5.77$ (s, H11); 4.24 (d, J13.13 Hz, 1H, H19); 4.16 (d, J $13.13 \mathrm{~Hz}, 1 \mathrm{H}$, H19'); 2.23 (s, 3H, $\mathrm{CH}_{3}$ ). ${ }^{13} \mathrm{C} \mathrm{NMR}$ (DMSO-d 6 , $75 \mathrm{MHz}$ ): $\delta$ (ppm) 184.1, 179.9, 171.5, 153.2, 134.6, 133.9, 132.8, 131.7, 131.2, 130.1, 129.9, 128.9, 128.7, 128.6, 127.6, 125.6, 125.3, 123.5, 116.0, 110.2, 54.0, 49.5, 20.4. UV-Vis $\left(\mathrm{CHCl}_{3}\right) \lambda / \mathrm{nm}$, $\log \varepsilon: 250$ (4.46), 310 (4.15), 382 (3.90).

3-[N-(n-butyl)amino-2-hydroxy-5-bromo-benzyl]-2hydroxy-1,4-naphthoquinone (HL12)

From butylamine $(0.54 \mathrm{~mL})$ and 2-hydroxy-5-bromobenzaldehyde (1.206 g). Yield: $1.316 \mathrm{~g}, 61 \%$; mp 166$167{ }^{\circ} \mathrm{C}$. Anal. Calc. for $\mathrm{C}_{21} \mathrm{H}_{20} \mathrm{BrNO}_{4}$ : C, 58.62; H, 4.68; $\mathrm{N}, 3.26$. Found: C, 58.74; H, 4.54; N, 3.42\%. IR (KBr) $\mathrm{v}_{\max } / \mathrm{cm}^{-1}: 3202 ; 2961 ; 2934 ; 2867 ; 1679 ; 1591 ; 1524 ; 1273$; 1229. ${ }^{1} \mathrm{H}$ NMR (DMSO-d ${ }^{6}, 300 \mathrm{MHz}: \delta$ (ppm) 8.04 (d, $J$ $7.20 \mathrm{~Hz}, 1 \mathrm{H}, H 5$ or H8); 7.98 (d, J 7.20 Hz, 1H, H8 or H5); 7.84 (t, J 7.20, $7.20 \mathrm{~Hz}, 1 \mathrm{H}, H 6$ or $H 7$ ); 7.73 (t, J 7.20, 7.20 $\mathrm{Hz}, 1 \mathrm{H}, H 7$ or H6); 7.64 (d, J $2.15 \mathrm{~Hz}, 1 \mathrm{H}, H 17) ; 7.42$ (dd, J 8.58, 2.15 Hz, 1H, H15); 6.94 (d, J 8.58 Hz, 1H, H14); 5.82 (s, $1 \mathrm{H}, H 11) ; 3.05-2.95$ (m, 2H, H19); 1.78-1.65 (m, 2H, H2O); 1.49-1.35 (m, 2H, H21); 0.96 (t, J 7.04, 7.04 Hz, 3H, H22). ${ }^{13} \mathrm{C} \mathrm{NMR}$ (DMSO-d 6 , $75 \mathrm{MHz}$ ): $\delta$ (ppm) 183.9, 179.5, 171.1, 154.8, 134.4, 133.9, 132.0, 131.6, 131.2, 130.9, 126.5, 125.6, $125.2,118.2,110.2,109.8,53.4,45.8,27.8,19.3,13.6$. UV-Vis $\left(\mathrm{CHCl}_{3}\right) \lambda / \mathrm{nm}, \log \varepsilon: 273$ (3.12), 354 (2.27), 457 (2.05).

3-[N-(benzyl)amino-2-hydroxy-5-bromo-benzyl]-2hydroxy-1,4-naphthoquinone (HL13)

From benzylamine $(0.60 \mathrm{~mL})$ and 2-hydroxy-5-bromobenzaldehyde (1.206 g). Yield: $1.736 \mathrm{~g}, 75 \%$; mp 160$161{ }^{\circ} \mathrm{C}$. Anal. Calc. for $\mathrm{C}_{24} \mathrm{H}_{18} \mathrm{BrNO}_{4}$ : C, 62.08; H, 3.91; N, 3.02. Found: C, 62,09; H, 4.01; N, 3.11\%. IR (KBr) $v_{\max } / \mathrm{cm}^{-1}$ : 3224 (O-H), 3068 (C-H), 2958 (C-H), 1688 (C=O), 1589 
$(\mathrm{C}=\mathrm{C}), 1539(\delta \mathrm{N}-\mathrm{H}), 1275(\mathrm{C}-\mathrm{O}) .{ }^{1} \mathrm{H}$ NMR $\left(\mathrm{DMSO}^{\mathrm{d}} \mathrm{d}^{6}, 300\right.$ MHz): $\delta$ (ppm) 8.04 (dd, $J 7.62,1.36 \mathrm{~Hz}, 1 \mathrm{H}, H 5$ or $H 8$ ); 7.99 (d, $J$ 7.54, $1.41 \mathrm{~Hz}, 1 \mathrm{H}, H 8$ or H5); 7.85 (td, $J$ 7.44, $7.44,1.41 \mathrm{~Hz}, 1 \mathrm{H}, H 6$ or $H 7) ; 7.74$ (td, J 7.44, 7.44, $1.36 \mathrm{~Hz}$, $1 \mathrm{H}, H 7$ or $H 6) ; 7.54(\mathrm{~d}, J 2.37 \mathrm{~Hz}, 1 \mathrm{H}, H 17) ; 7.50-7.46$ (m, $5 \mathrm{H}, P h) ; 7.39$ (dd, J 8.63, $2.37 \mathrm{~Hz}, 1 \mathrm{H}, H 15) ; 6.89$ (d, J 8.63 $\mathrm{Hz}, 1 \mathrm{H}, H 14) ; 5.77$ (s, 1H, H11); 4.24 (d, J $13.15 \mathrm{~Hz}, 1 \mathrm{H}$, H19); 4.15 (d, J $\left.13.15 \mathrm{~Hz}, 1 \mathrm{H}, H 19^{\prime}\right) .{ }^{13} \mathrm{C}$ NMR (DMSO-d ${ }^{6}$, $75 \mathrm{MHz}) \delta$ (ppm): 184.5, 180.3, 172.1, 155.6, 135.1, 134.5, 133.3, 132.5, 132.3, 131.8, 131.5, 130.7, 129.5, 129.3, 129.1, $127.1,126.2,125.9,118.8,110.7,110.4,53.9,50.1$. UV-Vis $\left(\mathrm{CHCl}_{3}\right) \lambda / \mathrm{nm}, \log \varepsilon: 271$ (3.10), 377 (2.60), 434 (1.94).

Synthesis of complexes $\left[\mathrm{Cu}(\mathrm{L})_{2}\right]$ 1-13 from HL1-HL13, respectively

To a suspension of $1 \mathrm{mmol}$ of the ligand in $10 \mathrm{~mL}$ $\mathrm{MeOH}$, was added a solution of $\mathrm{CuCl}_{2} 2 \mathrm{H}_{2} \mathrm{O}(83 \mathrm{mg}$, $0.5 \mathrm{mmol})$ in $2 \mathrm{~mL} \mathrm{MeOH}$. After addition of $\mathrm{Et}_{3} \mathrm{~N}(0.14 \mathrm{~mL}$, $1 \mathrm{mmol}$ ), the suspension was left under stirring in the dark for $12 \mathrm{~h}$ at room temperature. The resulting solids were filtered off, washed with methanol, diethyl ether and dried under vacuum (Figure 2).

\section{$\left[\mathrm{Cu}(\mathbf{L 1})_{2}\right](\mathbf{1})$}

From 335 mg of HL1. Yield: $337 \mathrm{mg}, 92 \%$; mp $198^{\circ} \mathrm{C}$. Slow evaporation of a $\mathrm{CHCl}_{3}$ solution yielded brown crystals suitable for X-ray diffraction analysis. Anal. calc. for $\mathrm{C}_{42} \mathrm{H}_{40} \mathrm{~N}_{2} \mathrm{O}_{6} \mathrm{Cu} \cdot 2 \mathrm{H}_{2} \mathrm{O}: \mathrm{C}, 65.65 ; \mathrm{H}, 5.77 ; \mathrm{N}, 3.65 \%$. Found: C, 64.69; H, 5.76; N, 3.54\%. IR (KBr) $v_{\max } / \mathrm{cm}^{-1}$ : $3468(\mathrm{O}-\mathrm{H}), 3281(\mathrm{~N}-\mathrm{H}), 3064(\mathrm{C}-\mathrm{H}), 2958(\mathrm{C}-\mathrm{H}), 2928$ (C-H), $1674(\mathrm{C}=\mathrm{O}), 1621(\mathrm{C}=\mathrm{C}), 1591(\mathrm{C}=\mathrm{C}), 1273(\mathrm{C}-$ O). UV-Vis $\left(\mathrm{CHCl}_{3}\right) \lambda / \mathrm{nm}, \log \varepsilon$ : 315 (4.13), 425 (3.78), 538 (2.25).

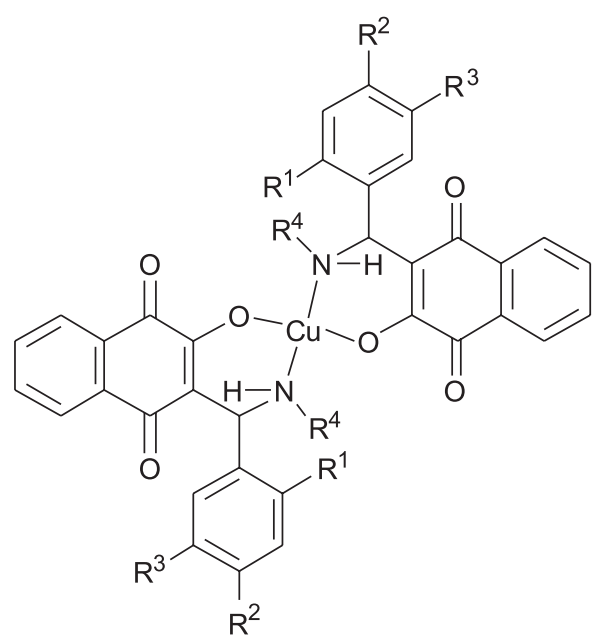

[Cu( $\left.\mathbf{L 2})_{2}\right](2)$

From $380 \mathrm{mg}$ of HL2. Yield: $280 \mathrm{mg}, 68 \%$; mp $208^{\circ} \mathrm{C}$. Slow evaporation of the complex solution in a methanol/ isopropanol mixture yielded brown crystals suitable for X-ray diffraction analysis. Anal. calc. for $\mathrm{C}_{42} \mathrm{H}_{38} \mathrm{~N}_{4} \mathrm{O}_{10} \mathrm{Cu}$. $\mathrm{H}_{2} \mathrm{O}: \mathrm{C}, 60.03 ; \mathrm{H}, 4.80 ; \mathrm{N}, 6.67 \%$. Found: $\mathrm{C}, 59.21 ; \mathrm{H}, 4.70$; $\mathrm{N}, 6.82 \%$. IR $(\mathrm{KBr}) \mathrm{v}_{\max } / \mathrm{cm}^{-1}: 3460(\mathrm{O}-\mathrm{H}), 3273(\mathrm{~N}-\mathrm{H}), 3077$ (C-H), 2956 (C-H), $2930(\mathrm{C}-\mathrm{H}), 1675(\mathrm{C}=\mathrm{O}), 1617(\mathrm{C}=\mathrm{C})$, $1592(\mathrm{C}=\mathrm{C}), 1546(\delta \mathrm{N}-\mathrm{H}), 1272(\mathrm{C}-\mathrm{O})$. UV-Vis $\left(\mathrm{CHCl}_{3}\right)$ $\lambda / \mathrm{nm}, \log \varepsilon$ : 298 (4.64), 324 (4.05), 415 (3.72), 536 (2.17).

$\left[\mathrm{Cu}(\mathbf{L 3})_{2}\right](\mathbf{3})$

From $414 \mathrm{mg}$ of HL3. Yield: $280 \mathrm{mg}, 63 \%$; mp 176$177{ }^{\circ} \mathrm{C}$. Anal. Calc. for $\mathrm{C}_{48} \mathrm{H}_{34} \mathrm{~N}_{4} \mathrm{O}_{10} \mathrm{Cu} \cdot 2 \mathrm{H}_{2} \mathrm{O}$ : C, 62.23; $\mathrm{H}, 4.13$; N, 6.05\%. Found: C, 61.19; H, 4.12; N, 6.16\%. IR (KBr) $v_{\max } / \mathrm{cm}^{-1}: 3459(\mathrm{O}-\mathrm{H}), 3151(\mathrm{~N}-\mathrm{H}), 2933(\mathrm{C}-\mathrm{H})$, $1671(\mathrm{C}=\mathrm{O}), 1591(\mathrm{C}=\mathrm{C}), 1547(\delta \mathrm{N}-\mathrm{H}), 1274(\mathrm{C}-\mathrm{O})$. UV-Vis $\left(\mathrm{CHCl}_{3}\right) \lambda / \mathrm{nm}, \log \varepsilon$ : 298 (4.51), 319 (4.16), 413 (3.70), 556 (2.17).

\section{$\left[\mathrm{Cu}(\mathbf{L 4})_{2}\right](\mathbf{4})$}

From $404 \mathrm{mg}$ of HL4. Yield: $322 \mathrm{mg}, 74 \%$; $\mathrm{mp} 171^{\circ} \mathrm{C}$. Anal. Calc. for $\mathrm{C}_{42} \mathrm{H}_{36} \mathrm{Cl}_{4} \mathrm{~N}_{2} \mathrm{O}_{6} \mathrm{Cu} \cdot 0.5 \mathrm{H}_{2} \mathrm{O}: \mathrm{C}, 57.38 ; \mathrm{H}, 4.24$; $\mathrm{N}, 3.19 \%$. Found: C, 56.49; H, 4.28; N, 3.28\%. IR (KBr) $v_{\max } / \mathrm{cm}^{-1}: 3446(\mathrm{O}-\mathrm{H}) ; 3273(\mathrm{~N}-\mathrm{H}), 2959(\mathrm{C}-\mathrm{H}), 2931(\mathrm{C}-$ $\mathrm{H}) ; 2871(\mathrm{C}-\mathrm{H}), 1678(\mathrm{C}=\mathrm{O}), 1625(\mathrm{C}=\mathrm{C}), 1591(\mathrm{C}=\mathrm{C})$, $1548(\delta \mathrm{N}-\mathrm{H}), 1275(\mathrm{C}-\mathrm{O})$. UV-Vis $\left(\mathrm{CHCl}_{3}\right) \lambda / \mathrm{nm}, \log \varepsilon$ : 289 (4.40), 318 (3.97), 412 (3.70), 530 (2.22).

\section{$\left[\mathrm{Cu}(\mathbf{L 5})_{2}\right](\mathbf{5})$}

From $438 \mathrm{mg}$ of HL5. Yield: $276 \mathrm{mg}, 59 \%$; mp 172$173{ }^{\circ} \mathrm{C}$. Anal. Calc. for $\mathrm{C}_{48} \mathrm{H}_{32} \mathrm{Cl}_{4} \mathrm{~N}_{2} \mathrm{O}_{6}$ Cu. $\mathrm{H}_{2} \mathrm{O}: \mathrm{C}, 60.30$; H, 3.58; N, 2.93\%. Found: C, 58.87; H, 3.56; N, 3.02\%. IR (KBr) $v_{\text {max }} / \mathrm{cm}^{-1}: 3428(\mathrm{O}-\mathrm{H}), 3266(\mathrm{~N}-\mathrm{H}), 3066(\mathrm{C}-\mathrm{H})$,

$$
\begin{aligned}
& \mathrm{R}^{1}=\mathrm{R}^{2}=\mathrm{R}^{3}=\mathrm{H} ; \mathrm{R}^{4}=\mathrm{Bu}, \mathbf{1} \\
& \mathrm{R}^{1}=\mathrm{R}^{3}=\mathrm{H} ; \mathrm{R}^{2}=\mathrm{NO}_{2} ; \mathrm{R}^{4}=\mathrm{Bu}, \mathbf{2} \\
& \mathrm{R}^{1}=\mathrm{R}^{3}=\mathrm{H} ; \mathrm{R}^{2}=\mathrm{NO}_{2} ; \mathrm{R}^{4}=\mathrm{Bz}, \mathbf{3} \\
& \mathrm{R}^{1}=\mathrm{R}^{2}=\mathrm{Cl} ; \mathrm{R}^{3}=\mathrm{H} ; \mathrm{R}^{4}=\mathrm{Bu}, \mathbf{4} \\
& \mathrm{R}^{1}=\mathrm{R}^{2}=\mathrm{Cl} ; \mathrm{R}^{3}=\mathrm{H} ; \mathrm{R}^{4}=\mathrm{Bz}, \mathbf{5} \\
& \mathrm{R}^{1}=\mathrm{OH} ; \mathrm{R}^{2}=\mathrm{R}^{3}=\mathrm{H} ; \mathrm{R}^{4}=\mathrm{alyl}, \mathbf{6} \\
& \mathrm{R}^{1}=\mathrm{OH} ; \mathrm{R}^{2}=\mathrm{R}^{3}=\mathrm{H} ; \mathrm{R}^{4}=\mathrm{Bu}, \mathbf{7} \\
& \mathrm{R}^{1}=\mathrm{OH} ; \mathrm{R}^{2}=\mathrm{R}^{3}=\mathrm{H} ; \mathrm{R}^{4}=\mathrm{Bz}, \mathbf{8} \\
& \mathrm{R}^{1}=\mathrm{OH} ; \mathrm{R}^{2}=\mathrm{R}^{3}=\mathrm{H} ; \mathrm{R}^{4}=\mathrm{CH}_{2} \mathrm{C}_{4} \mathrm{H}_{3} \mathrm{O}, \mathbf{9} \\
& \mathrm{R}^{1}=\mathrm{OH} ; \mathrm{R}^{2}=\mathrm{H} ; \mathrm{R}^{3}=\mathrm{Me} ; \mathrm{R}^{4}=\mathrm{Bu}, 10 \\
& \mathrm{R}^{1}=\mathrm{OH} ; \mathrm{R}^{2}=\mathrm{H} ; \mathrm{R}^{3}=\mathrm{Me} ; \mathrm{R}^{4}=\mathrm{Bz}, \mathbf{1 1} \\
& \mathrm{R}^{1}=\mathrm{OH} ; \mathrm{R}^{2}=\mathrm{H} ; \mathrm{R}^{3}=\mathrm{Br} ; \mathrm{R}^{4}=\mathrm{Bu}, \mathbf{1 2} \\
& \mathrm{R}^{1}=\mathrm{OH} ; \mathrm{R}^{2}=\mathrm{H} ; \mathrm{R}^{3}=\mathrm{Br} ; \mathrm{R}^{4}=\mathrm{Bz}, 13
\end{aligned}
$$


$2926(\mathrm{C}-\mathrm{H}), 1676(\mathrm{C}=\mathrm{O}), 1625(\mathrm{C}=\mathrm{C}), 1591(\mathrm{C}=\mathrm{C}), 1549$ $(\delta \mathrm{N}-\mathrm{H}), 1277(\mathrm{C}-\mathrm{O}) . \mathrm{UV}-\mathrm{Vis}\left(\mathrm{CHCl}_{3}\right) \lambda / \mathrm{nm}, \log \varepsilon: 298$ (4.43), 320 (4.03), 411 (3.73), 553 (2.16).

\section{$\left[\mathrm{Cu}(\mathbf{L 6})_{2}\right](\mathbf{6})$}

From $335 \mathrm{mg}$ of HL6. Yield: $296 \mathrm{mg}, 81 \%$; mp > $310{ }^{\circ} \mathrm{C}$. Anal. Calc. for $\mathrm{C}_{40} \mathrm{H}_{32} \mathrm{~N}_{2} \mathrm{O}_{8} \mathrm{Cu} \cdot 0.5 \mathrm{H}_{2} \mathrm{O}: \mathrm{C}, 64.81$; H, 4.49; N, 3.78\%. Found: C, 63.29; H, 4.60; N, $4.01 \%$. IR $(\mathrm{KBr}) v_{\max } / \mathrm{cm}^{-1}: 3478(\mathrm{O}-\mathrm{H}), 3153(\mathrm{~N}-\mathrm{H}), 1670(\mathrm{C}=\mathrm{O})$, $1593(\mathrm{C}=\mathrm{O}), 1534(\delta \mathrm{N}-\mathrm{H}), 1279(\mathrm{C}-\mathrm{O})$. UV-Vis (DMSO) $\lambda / \mathrm{nm}, \log \varepsilon: 276$ (4.65), 454 (3.63).

\section{$\left[\mathrm{Cu}(\mathbf{L 7})_{2}\right](\mathbf{7})$}

From $351 \mathrm{mg}$ of HL7. Yield: $306 \mathrm{mg}, 80 \%$; mp $201^{\circ} \mathrm{C}$. Slow evaporation of the complex solution in THF/dioxane yielded brown crystals suitable for $\mathrm{X}$-ray diffraction analysis. Anal. Calc. for $\mathrm{C}_{42} \mathrm{H}_{40} \mathrm{~N}_{2} \mathrm{O}_{8} \mathrm{Cu} \cdot 2 \mathrm{H}_{2} \mathrm{O}: \mathrm{C}, 63.03 ; \mathrm{H}$, 5.54 ; N, 3.50\%. Found: C, 62.40; H, 5.63; N, 3.41\%. IR $(\mathrm{KBr}) v_{\max } / \mathrm{cm}^{-1}: 3260(\mathrm{~N}-\mathrm{H}), 2954(\mathrm{C}-\mathrm{H}), 2866(\mathrm{C}-\mathrm{H}), 1683$ $(\mathrm{C}=\mathrm{O}), 1593(\mathrm{C}=\mathrm{C}), 1530(\delta \mathrm{N}-\mathrm{H}), 1276(\mathrm{C}-\mathrm{O})$. UV-Vis (DMSO) $\lambda / \mathrm{nm}, \log \varepsilon: 277$ (4.58), 455 (3.58).

\section{$\left[\mathrm{Cu}(\mathbf{L 8})_{2}\right](8)$}

From $385 \mathrm{mg}$ of 8. Yield: $287 \mathrm{mg}, 69 \%$; mp $187^{\circ} \mathrm{C}$. Anal. Calc. for $\mathrm{C}_{48} \mathrm{H}_{36} \mathrm{~N}_{2} \mathrm{O}_{8} \mathrm{Cu} 2 \mathrm{H}_{2} \mathrm{O}: \mathrm{C}, 66.39 ; \mathrm{H}, 4.64 ; \mathrm{N}$,

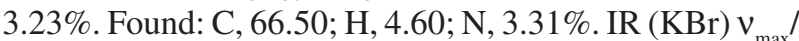
$\mathrm{cm}^{-1}$ : 3474 (O-H), 3274 (N-H), 3067 (C-H), 2944 (C-H), $1668(\mathrm{C}=\mathrm{O}), 1591(\mathrm{C}=\mathrm{C}), 1533(\delta \mathrm{N}-\mathrm{H}), 1279(\mathrm{C}-\mathrm{O})$. UVVis (DMSO) $\lambda / \mathrm{nm}, \log \varepsilon: 276$ (4.63), 449 (3.64).

\section{$\left[\mathrm{Cu}(\mathbf{L 9})_{2}\right](\mathbf{9})$}

From $375 \mathrm{mg}$ of 9 . Yield: $260 \mathrm{mg}, 64 \%$; $\mathrm{mp}>310^{\circ} \mathrm{C}$. Anal. Calc. for $\mathrm{C}_{44} \mathrm{H}_{32} \mathrm{~N}_{2} \mathrm{O}_{10} \mathrm{Cu} \cdot 1.5 \mathrm{H}_{2} \mathrm{O}: \mathrm{C}, 62.97 ; \mathrm{H}, 4.20$; N, 3.34\%. Found: C, 61.93; H, 4.01; N, 3.25\%. IR (KBr) $\mathrm{v}_{\max } / \mathrm{cm}^{-1}: 3478(\mathrm{O}-\mathrm{H}), 3245(\mathrm{~N}-\mathrm{H}), 1669(\mathrm{C}=\mathrm{O}), 1591$ $(\mathrm{C}=\mathrm{C}), 1534(\delta \mathrm{N}-\mathrm{H}), 1281(\mathrm{C}-\mathrm{O})$. UV-Vis (DMSO) $\lambda /$ nm, $\log \varepsilon: 276$ (4.65), 449 (3.61).

\section{$\left[\mathrm{Cu}(\mathrm{L10})_{2}\right],(\mathbf{1 0})$}

From $365 \mathrm{mg}$ of $\mathbf{1 0}$. Yield: $277 \mathrm{mg}, 70 \%$; mp $218^{\circ} \mathrm{C}$. Anal. Calc. for $\mathrm{C}_{44} \mathrm{H}_{44} \mathrm{~N}_{2} \mathrm{O}_{8} \mathrm{Cu}$ : C, 66.69; H, 5.60; N, 3.54\%. Found: C, 66.27; H, 5.64; N, 3.67\%. IR (KBr) $v_{\max } / \mathrm{cm}^{-1}$ : 3254 (N-H), 2954 (C-H), 2868 (C-H), 1693 (C=O), 1590 (C-C), $1500(\delta \mathrm{N}-\mathrm{H}), 1277(\mathrm{C}-\mathrm{O}) . \mathrm{UV}-\mathrm{Vis}(\mathrm{DMSO}) \lambda / \mathrm{nm}$, $\log \varepsilon: 277$ (4.64), 452 (3.69).

\section{$\left[\mathrm{Cu}(\mathrm{L11})_{2}\right](\mathbf{1 1})$}

From $399 \mathrm{mg}$ of $\mathbf{1 1}$. Yield: $330 \mathrm{mg}, 77 \%$; mp $196^{\circ} \mathrm{C}$. Anal. Calc. for $\mathrm{C}_{50} \mathrm{H}_{40} \mathrm{~N}_{2} \mathrm{O}_{8} \mathrm{Cu} . \mathrm{H}_{2} \mathrm{O}: \mathrm{C}, 68.37 ; \mathrm{H}, 4.82 ; \mathrm{N}$, $3.19 \%$. Found: C, 67.21; H, 4.68; N, 3.29\%. IR (KBr) $\mathrm{v}_{\max }$ ' $\mathrm{cm}^{-1}$ : $3400(\mathrm{O}-\mathrm{H}) ; 3251(\mathrm{~N}-\mathrm{H}) ; 3028(\mathrm{C}-\mathrm{H}), 2918(\mathrm{C}-\mathrm{H})$,
$1674(\mathrm{C}=\mathrm{O}), 1590(\mathrm{C}=\mathrm{C}), 1535(\delta \mathrm{N}-\mathrm{H}), 1279(\mathrm{C}-\mathrm{O})$. UVVis (DMSO) $\lambda / \mathrm{nm}, \log \varepsilon: 276$ (4.63), 447 (3.63).

[Cu(L12) $)_{2}$ (12)

From $430 \mathrm{mg}$ of HL12. Yield: $152 \mathrm{mg}$, 33\%; mp 186$187^{\circ} \mathrm{C}$. Anal. Calc. for $\mathrm{C}_{42} \mathrm{H}_{38} \mathrm{Br}_{2} \mathrm{~N}_{2} \mathrm{O}_{8} \mathrm{Cu} \cdot 1.5 \mathrm{H}_{2} \mathrm{O}: \mathrm{C}, 53.15$; H, 4.35; N, 2.95\%. Found: C, 51.75; H, 4.22; N, 3.10\%. IR $(\mathrm{KBr}) v_{\max } / \mathrm{cm}^{-1}: 3422(\mathrm{O}-\mathrm{H}), 3253(\mathrm{~N}-\mathrm{H}), 2959(\mathrm{C}-\mathrm{H})$, 2933 (C-H), 2869 (C-H), 1675 (C=O), 1589 (C=C), 1532 $(\delta \mathrm{N}-\mathrm{H}), 1277(\mathrm{C}-\mathrm{O})$. UV-Vis $\left(\mathrm{CHCl}_{3}\right) \lambda / \mathrm{nm}, \log \varepsilon: 276$ (4.59), 347 (3.58), 428 (3.58).

$\left[\mathrm{Cu}(\mathrm{L13})_{2}\right](\mathbf{1 3})$

From $464 \mathrm{mg}$ of HL13. Yield: $294 \mathrm{mg}, 60 \%$; mp 202$203^{\circ} \mathrm{C}$. Anal. Calc. for $\mathrm{C}_{48} \mathrm{H}_{34} \mathrm{Br}_{2} \mathrm{~N}_{2} \mathrm{O}_{8} \mathrm{Cu} \cdot 2 \mathrm{H}_{2} \mathrm{O}: \mathrm{C}, 56.18$; H, 3.73; N, 2.73\%. Found: C, 55.77; H, 3.67; N, $2.80 \%$. IR (KBr) $v_{\max } / \mathrm{cm}^{-1}: 3454(\mathrm{O}-\mathrm{H}), 3213(\mathrm{~N}-\mathrm{H}), 2946(\mathrm{C}-\mathrm{H})$, $1673(\mathrm{C}=\mathrm{O}), 1591(\mathrm{C}=\mathrm{C}), 1531(\delta \mathrm{N}-\mathrm{H}), 1279(\mathrm{C}-\mathrm{O})$. UV-Vis (DMSO) $\lambda / \mathrm{nm}, \log \varepsilon: 276$ (4.65), 450 (3.65).

$X$-ray crystallography

The $x$-ray diffraction data for compounds were collected using a Bruker KAPPA CCD diffractometer, ${ }^{31}$ at $295 \mathrm{~K}$ and Mo graphite monochromatic radiation. The cell parameters for the molecules were obtained and refined using the $\mathrm{PHICHI}^{32}$ and DIRAX ${ }^{33}$ programs, respectively, catching reflections with random orientation in hkl planes. Intensities were corrected by Lorentz polarization and absorption with the SADABS program. ${ }^{34}$ The structure was solved by Direct Methods using the SHELXS-97 program. ${ }^{35}$ The anisotropy parameters of non-H atoms were refined with the SHELXL-97 program. ${ }^{36}$ In 1, 2 and 7 the aromatic, methyl, methyne and methylene $\mathrm{H}$-atoms were geometrically included in the refinement. Aromatic carbons were refined with $\mathrm{U}_{\text {iso }}(\mathrm{H})=1.2 \mathrm{Ueq}$ $\mathrm{Csp}^{2}$, methylene carbons with $\mathrm{U}_{\text {iso }}(\mathrm{H})=1.2 \mathrm{Ueq} \mathrm{Csp}^{3}$, methine carbons with $\mathrm{U}_{\text {iso }}(\mathrm{H})=1.2 \mathrm{Ueq} \mathrm{Csp}^{3}$ and methyl carbons with $\mathrm{U}_{\text {iso }}(\mathrm{H})=1.5 \mathrm{Ueq} \mathrm{Csp}^{3}$. The hydrogen atom of the water molecules, $\mathrm{N}-\mathrm{H}$ amine in the three compounds and $\mathrm{O}-\mathrm{H}$ hydroxyl for $\mathbf{2}$ were localized experimentally in the Fourier map. For $\mathbf{2}$ the hydrogen atom coordinates corresponding to a water molecule could not be localized experimentally in the Fourier map. In view of this we opted for using the SQUEEZE ${ }^{37}$ tool contained in the WinGX ${ }^{38}$ package, in order to exclude any electronic density contributions relative to the disordered water molecules. This procedure is in accordance with the elemental analysis of the complex, confirming a species free from any crystallization solvate. Consequently we do not comment in this work on the hydrogen bonds for 2 . 
The solution and refinement of $\mathbf{1}$ suggested the presence of disordered C21 carbon of the butyl moiety. X-ray data are listed in Table 1 and ORTEP- $3^{39}$ for Windows was used to draw the Figures.

\section{Antibacterial assays}

The antibacterial evaluation was performed with Gram-positive (Bacillus cereus ATCC 33019, Bacillus subtilis ATCC 6633, Enterococcus faecalis ATCC 29212, Staphylococcus aureus ATCC 25923) and Gram-negative (Escherichia coli ATCC 25922, Klebsiella pneumoniae ATCC 700603, Pseudomonas aeruginosa ATCC 27853) bacteria as test-microorganisms.
Minimum inhibitory concentration (MIC) was determined by the microdilution broth technique according to the M7-A6 document. ${ }^{40}$ The assays were carried in 96well tissue culture microplates filled with Mueller Hinton broth $\left(100 \mu \mathrm{L}\right.$ per well) ${ }^{41}$ The inoculum suspension of each strain was prepared in Mueller Hinton broth (108 bacteria cells per $\mathrm{mL}$, corresponding to $\mathrm{O}$. D. $=0.08-0.1$ at $625 \mathrm{~nm}$ ) and diluted to 1:10. All samples were tested in eighth concentrations from 3 to $0.02 \times 10^{-3} \mathrm{~mol} \mathrm{~L}^{-1}$. The inoculum suspension ( $5 \mu \mathrm{L}$ per well) was applied into the microplates which were incubated at $37{ }^{\circ} \mathrm{C}$ overnight. An aqueous solution of $p$-iodonitrotetrazolium violet (p-INT) (Sigma) $(20 \mu \mathrm{L})$ was added ${ }^{42}$ and the microplates were incubated once more for $1-2$ hours at $37{ }^{\circ} \mathrm{C}$. The

Table 1. Crystallographic data and refinement parameters for $\mathbf{1 , 2}$ and $\mathbf{7}$

\begin{tabular}{|c|c|c|c|}
\hline Formula & $\mathrm{C}_{42} \mathrm{H}_{40} \mathrm{~N}_{2} \mathrm{O}_{6} \mathrm{Cu} \cdot 2 \mathrm{H}_{2} \mathrm{O}(\mathbf{1})$ & $\mathrm{C}_{42} \mathrm{H}_{38} \mathrm{~N}_{4} \mathrm{O}_{10} \mathrm{Cu} \cdot 2 \mathrm{C}_{3} \mathrm{H}_{8} \mathrm{O}_{1}(2)$ & $\mathrm{C}_{42} \mathrm{H}_{40} \mathrm{~N}_{2} \mathrm{O}_{8} \mathrm{Cu} \cdot \mathrm{H}_{8} \mathrm{C}_{4} \mathrm{O}_{2} \cdot 2 \mathrm{H}_{2} \mathrm{O}(7)$ \\
\hline Formula weight & 768.37 & 942.49 & 888.44 \\
\hline $\mathrm{T} / \mathrm{K}$ & 295 & 295 & 295 \\
\hline Radiation, $\lambda / \AA$ & 0.71073 & 0.71073 & 0.71073 \\
\hline Crystal System, space group & Triclinic, $P-1$ & Monoclinic, $C 2 / \mathrm{c}$ & Monoclinic, $P 2_{1} / n$ \\
\hline $\begin{array}{l}\text { Unit cell dimensions, } \\
a, b, c / \AA \\
\alpha, \beta, \gamma / \text { degree }\end{array}$ & $\begin{array}{l}a=9.473(2) \\
b=10.124(2) \\
c=11.984(2) \\
\alpha=107.30(3) \\
\beta=90.81(3) \\
\gamma=117.02(3)\end{array}$ & $\begin{array}{l}a=26.187(5) \\
b=10.382(2) \\
c=21.158(4) \\
\beta=115.55(3)\end{array}$ & $\begin{array}{l}a=10.271(2) \\
b=17.274(4) \\
c=12.286(3) \\
\beta=97.97(3)\end{array}$ \\
\hline Volume / $\AA^{3}$ & $962.4(3)$ & $5189.6(18)$ & $2158.8(8)$ \\
\hline$Z$, Calculated density $/ \mathrm{g} \mathrm{cm}^{-3}$ & $1 / 1.326$ & $4 / 1.206$ & $2 / 1.367$ \\
\hline Absorption coefficient $/ \mathrm{mm}^{-1}$ & 0.622 & 0.481 & 0.572 \\
\hline$F(000)$ & 403 & 1980 & 934 \\
\hline Crystal size $/ \mathrm{mm}^{3}$ & $0.35 \times 0.08 \times 0.06$ & $0.30 \times 0.24 \times 0.17$ & $0.30 \times 0.24 \times 0.15$ \\
\hline Theta range / degree & 5.43 to 27.49 & 2.14 to 25.50 & 5.13 to 25.50 \\
\hline Index range & $\begin{array}{l}-10 \leq h \leq 12 \\
-13 \leq k \leq 13 \\
-15 \leq l \leq 15\end{array}$ & $\begin{array}{l}-31 \leq h \leq 29 \\
-11 \leq k \leq 12 \\
-24 \leq l \leq 25\end{array}$ & $\begin{array}{l}-12 \leq h \leq 10, \\
-20 \leq k \leq 20, \\
-14 \leq l \leq 14\end{array}$ \\
\hline Reflections collected & 14396 & 72031 & 20068 \\
\hline Independent reflections & $4361\left[R_{(i n t)}=0.0751\right]$ & $4815\left[R_{(i n t)}=0.0763\right]$ & $3979\left[R_{(i n t)}=0.0365\right]$ \\
\hline Completeness to theta max. & $98.6 \%$ & $99.6 \%$ & $99.0 \%$ \\
\hline Max. and min. transmission & 0.8116 and 0.9636 & 0.9228 and 0.8693 & 0.9192 and 0.8472 \\
\hline Refinement method & Full-matrix least-squares on $F^{2}$ & Full-matrix least-squares on $F^{2}$ & Full-matrix least-squares on $F^{2}$ \\
\hline Data / restraints / parameters & $4361 / 0 / 258$ & 4815 / 0 / 301 & $3979 / 0 / 277$ \\
\hline Goodness-of-fit on $F^{2}$ & 1.059 & 1.109 & 1.060 \\
\hline Final $R$ indices $[I>2 \operatorname{sigma}(I)]$ & $\begin{array}{l}R_{1}=0.0572 \\
w R_{2}=0.1473\end{array}$ & $\begin{array}{l}R_{1}=0.0608 \\
w R_{2}=0.1469\end{array}$ & $\begin{array}{l}R_{1}=0.0465 \\
w R_{2}=0.1246\end{array}$ \\
\hline$R$ indices (all data) & $\begin{array}{l}R_{1}=0.0851 \\
w R_{2}=0.1611\end{array}$ & $\begin{array}{l}R_{1}=0.0808 \\
w R_{2}=0.1567\end{array}$ & $\begin{array}{l}R_{1}=0.0685 \\
w R_{2}=0.1429\end{array}$ \\
\hline Extinction coefficient & none & $0.0002(2)$ & None \\
\hline Largest diff. peak and hole $\left(\mathrm{e}^{-} \AA^{-3}\right)$ & 0.538 and -0.762 & 0.397 and -0.219 & 0.900 and -0.722 \\
\hline
\end{tabular}


MIC was defined as the lowest concentration of the extracts that inhibited the antibacterial visible growth as indicated by the p-INT colorimetric reagent. For sterility and growth control the Mueller Hinton broth was used without solvent or compounds. All strains were subcultured twice to verify the cell viability. Tests were performed in triplicate.

\section{Results and Discussion}

\section{Syntheses}

The Mannich bases HL1-HL13 (Figure 1) were synthesized from the reactions of 2-hydroxy-1,4naphthoquinone (lawsone) with an various primary amines and aldehydes in ethanol under stirring at room temperature. The orange products are stable in the solid state, but undergo decomposition when left in solution for a long period of time. Compounds HL1-HL7 and HL11HL13 are obtained in a pure state, but HL8-HL10 need to be recrystallised from hot ethanol. They were obtained in yields ranging from 53 (HL4) to 93\% and formulated on the basis of analytical and spectroscopic data (see Experimental).

The ${ }^{1} \mathrm{H}$ spectra of compounds HL1-HL13 exhibit peaks due to the four naphthoquinone aromatic hydrogens H5H8 that appear in the $\delta 7-8 \mathrm{ppm}$ region as dd or ddd (H5 and $\mathrm{H} 8$ ) and td (H6 and H7) (see Figure 1 for numbering and experimental for data). The other chemical shifts are compatible with the structures proposed for these compounds. In general, the butyl hydrogens appear in the $\delta 0.9$ to $3.1 \mathrm{ppm}$ region as multiplets $(\mathrm{H} 19-\mathrm{H} 21)$ or triplets (methyl $\mathrm{H} 22$ ); in the case of the benzyl group; the $\mathrm{CH}_{2}$ hydrogens H19 appear as a doublet around $\delta 4.2-4.6$ and the phenyl $\mathrm{H} 21-\mathrm{H} 25$, as multiplets. The (substituted) phenyl group hydrogens H13-17 appear as expected, depending on the substitution pattern. Attributions were made on the basis of ${ }^{1} \mathrm{H} \times{ }^{1} \mathrm{H}$ (COSY experiments), $J$ values and multiplicity. All expected resonances were observed in the ${ }^{13} \mathrm{C}$ NMR spectra of compounds HL1-HL13. The resonances arising from the carbonyl carbons were found around $\delta 185$ and 179 , and those attributed to $\mathrm{C} 2$ bound to the hydroxyl group, at about $\delta 171$.

Complexes 1-13 (Figure 2) were obtained by addition of trietylamine to a methanolic suspension of the ligand and $\mathrm{CuCl}_{2}-2 \mathrm{H}_{2} \mathrm{O}(2: 2: 1)$, under stirring at room temperature for $12 \mathrm{~h}$ in yields varying from 60 to $92 \%$, except for complex 12, isolated in $30 \%$. Elemental analysis confirmed the proposed formulation. Due to low solubility in methanol, acetonitrile and water, conductivity measurements could not be carried out.
All compounds were also characterized by EPR and UV-Vis spectroscopy, and the structures of 1, 2 and 7, determined by X-ray diffraction analyses.

\section{Description of the X-ray structures}

Good quality crystals suitable for single crystal X-ray diffraction analyses were obtained for compounds $\mathbf{1}, \mathbf{2}$ and $\mathbf{7}$. The molecular structures of $\mathbf{1}, \mathbf{2}$ and $\mathbf{7}$ are shown in Figures 3, 4 and 5, respectively and selected bond lengths and angles are given in Table 2.

All complexes crystallize in centrosymmetric space groups, with a copper atom in the inversion centre. Two deprotonated ligands $\left(\mathbf{L}^{-}\right)$coordinate through the naphthalen-2-olate oxygen and secondary amine- $\mathrm{N}$ atoms, forming two six-membered chelate rings around the copper atom in a trans $-\mathrm{N}_{2} \mathrm{O}_{2}$ environment. Bond angles $\mathrm{O}(1)$ Cu-N(1) (and $\beta$ parameters: ${ }^{43} 88.43(9), 1,91.33(10), 2$, and $\left.90.67(9)^{\circ}, 7\right)$ and $\mathrm{Cu}-\mathrm{N}$ and $\mathrm{Cu}-\mathrm{O}$ distances indicate slightly distorted square-planar coordination of the complexes. The

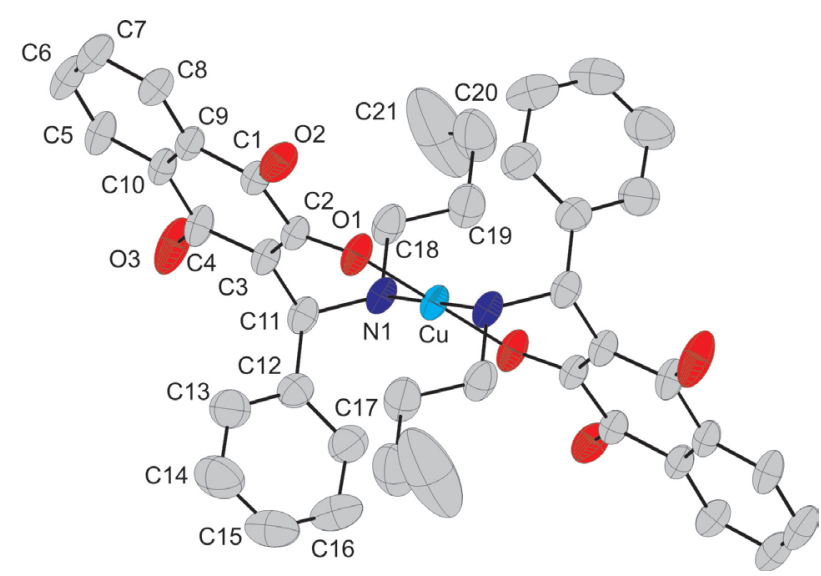

Figure 3. ORTEP view of $\left[\mathrm{Cu}(\mathbf{L 1})_{2}\right] \cdot 2 \mathrm{H}_{2} \mathrm{O} 1$ with labeled atoms and $50 \%$ probability ellipsoids; $\mathrm{H}$ atoms were omitted for the sake of clarity.

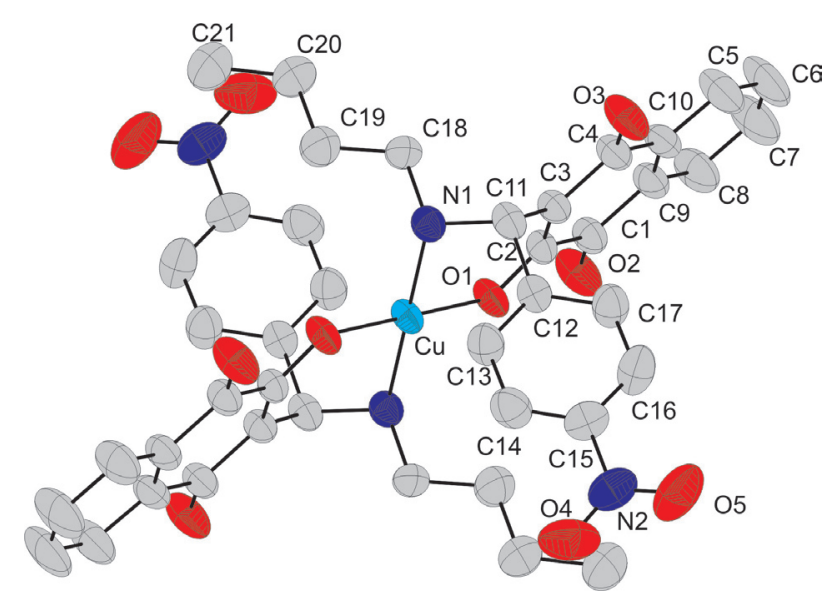

Figure 4. ORTEP view of $\left[\mathrm{Cu}(\mathbf{L 2})_{2}\right] \cdot \mathrm{H}_{2} \mathrm{O} 2$ with labeled atoms and $50 \%$ probability ellipsoids; $\mathrm{H}$ atoms were omitted for the sake of clarity. 


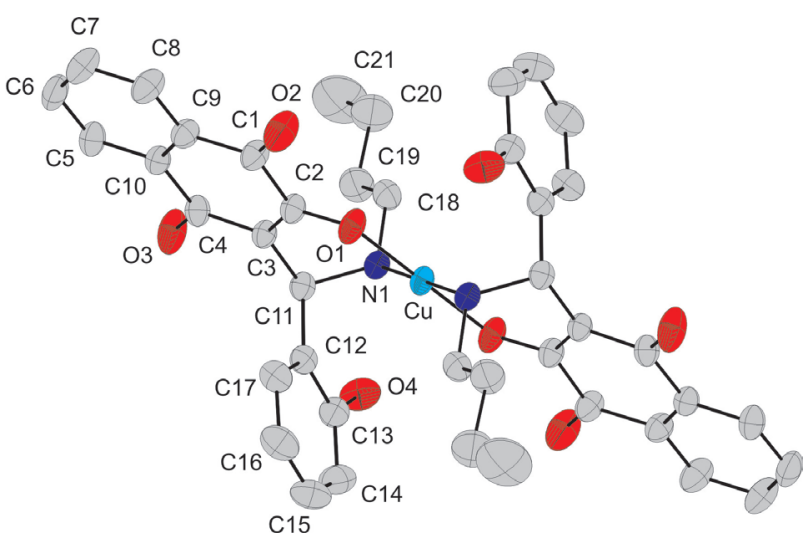

Figure 5. ORTEP view of $\left[\mathrm{Cu}(\mathbf{L} 7)_{2}\right] \cdot 2 \mathrm{H}_{2} \mathrm{O} 7$ with labeled atoms and $50 \%$ probability ellipsoids; $\mathrm{H}$ atoms were omitted for the sake of clarity.

Table 2. Selected bond distances $(\AA)$ and angles $\left(^{\circ}\right)$ for complexes 1, 2 and 7

\begin{tabular}{lccc}
\hline Bond distances / $\AA$ & $\mathbf{1}$ & $\mathbf{2}$ & $\mathbf{7}$ \\
\hline $\mathrm{Cu}-\mathrm{N}(1)$ & $2.023(2)$ & $2.023(3)$ & $1.996(2)$ \\
$\mathrm{Cu}-\mathrm{O}(1)$ & $1.945(2)$ & $1.928(2)$ & $1.942(2)$ \\
$\mathrm{N}(1)-\mathrm{C}(11)$ & $1.509(4)$ & $1.489(4)$ & $1.512(3)$ \\
$\mathrm{N}(1)-\mathrm{C}(18)$ & $1.510(4)$ & $1.492(4)$ & $1.495(4)$ \\
$\mathrm{O}(1)-\mathrm{C}(2)$ & $1.310(3)$ & $1.300(3)$ & $1.297(3)$ \\
$\mathrm{O}(2)-\mathrm{C}(1)$ & $1.230(4)$ & $1.216(4)$ & $1.223(4)$ \\
$\mathrm{O}(3)-\mathrm{C}(4)$ & $1.243(4)$ & $1.232(4)$ & $1.239(3)$ \\
\hline $\mathrm{Bond}$ angles / ( $)$ & & & \\
\hline $\mathrm{O}(1)-\mathrm{Cu}-\mathrm{N}(1)$ & $88.43(9)$ & $91.33(10)$ & $90.67(9)$ \\
$\mathrm{Cu}-\mathrm{N}(1)-\mathrm{C}(11)$ & $110.46(17)$ & $109.08(18)$ & $106.52(16)$ \\
$\mathrm{C}(3)-\mathrm{C}(11)-\mathrm{N}(1)$ & $109.0(3)$ & $110.6(2)$ & $110.0(2)$ \\
$\mathrm{C}(2)-\mathrm{C}(3)-\mathrm{C}(11)$ & $120.5(2)$ & $120.6(2)$ & $120.6(2)$ \\
$\mathrm{O}(1)-\mathrm{C}(2)-\mathrm{C}(3)$ & $125.4(2)$ & $125.8(3)$ & $125.6(2)$ \\
$\mathrm{C}(2)-\mathrm{O}(1)-\mathrm{Cu}$ & $126.86(19)$ & $127.19(18)$ & $126.29(18)$ \\
$\mathrm{C}(11)-\mathrm{N}(1)-\mathrm{C}(18)$ & $111.0(2)$ & $110.6(2)$ & $114.9(2)$ \\
\hline
\end{tabular}

$\mathrm{Cu}-\mathrm{N}_{\text {amine }}(2.023(2), 1,2.023(3), 2$, and 1.996(2) $\AA$, 7) and $\mathrm{Cu}-\mathrm{O}_{\text {phenolate }}$ distances (1.945(2), 1, 1.928(2), 2, and 1.942(2) $\AA$, 7) are in the normal range when compared to those observed for other copper(II) complexes containing the same coordination environment. ${ }^{44-46}$ In the same molecule, the two ligands have different absolute configurations at the chiral C11carbon. In the structures of compounds $\mathbf{1}$ and $\mathbf{2}$ the butyl and phenyl groups block the axial positions preventing further coordination to donor molecules (e.g. coordinating solvent), normally observed in the structures of analogous complexes. ${ }^{47,48}$

The packing arrangement of $\mathbf{1}$ exhibits molecules of $\mathbf{1}$ and water linked by $\mathrm{N}-\mathrm{H} \cdots \mathrm{O}$ and $\mathrm{O}-\mathrm{H} \cdots \mathrm{O}$ classic hydrogen bonds along the [100] crystallographic direction, resulting in a 1D supramolecular arrangement, Figure 6. The water molecules are responsible for the 1D self-assembly formed in the solid state, and the network is cemented by bifurcated and linear $\mathrm{H}$-bonds around these molecules. The $\mathrm{O}^{\#-}$

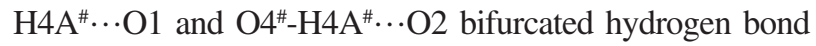
forms a five membered ring between complex 1 and water, these interactions providing stabilization of the 1D network [symmetry code for $(\mathbf{1}):(\#)=x, 1+y, z ;(\# \#)=1+x, y, z]$. In addition the crystalline structure is stabilized by $\mathrm{C}-\mathrm{H} \cdots \mathrm{O}$ and $\mathrm{C}-\mathrm{H} \cdots \mathrm{N}$ intramolecular interactions (Figure 7). Table 3 shows all H-bond parameters. All hydrogen bonds were calculated using PLATON ${ }^{37}$ (Table 3) and agree with the literature. ${ }^{37}$

The crystalline structure of complex 7 shows a 2D selfarrangement governed by $\mathrm{O}-\mathrm{H} \cdots \mathrm{O}$ and $\mathrm{C}-\mathrm{H} \cdots \mathrm{O}$ classical and non classical hydrogen bonds, respectively. The lattice grows in the [100] direction through $\mathrm{O}^{i i}{ }_{-} \mathrm{H}_{6} \mathrm{~A}^{i i} \ldots \mathrm{O} 3, \mathrm{O}^{i i_{-}}$ $\mathrm{H}_{6} \mathrm{~B}^{i i \cdots}{ }^{i \cdots}{ }^{i i i}$ and $\mathrm{O} 4{ }^{i}-\mathrm{H} 4^{i} \cdots \mathrm{O}^{i i}$ interactions and in the [001] direction, through $\mathrm{C} 18-\mathrm{H} 18 \mathrm{~B} \cdots \mathrm{O} 5^{i i i}$ interactions, as shown in Figure 8 [symmetry code for 7: $(i)=-1-x,-y, 1-z$; (ii) $=-x, 1-y, 1-z ;($ iii $)=-1+x, y, z$, Table 4]. The network in the [100] direction is built around the water molecules and in the [001] direction, via the dioxane interactions. In addition, intramolecular interactions via $\mathrm{N}-\mathrm{H} \cdots \mathrm{O}$ and $\mathrm{C}-\mathrm{H} \cdots \mathrm{O}$, specifically the $\mathrm{N} 1-\mathrm{H} 1 \cdots \mathrm{O} 4$ hydrogen bond prevents the copper atom from interacting with the hydroxyl $\mathrm{O}(4)$, as illustrated in Figure 9. Similar type of interaction has been observed in an analogous copper compound. ${ }^{45}$ The geometric parameters for these interactions are listed in Table 4. All hydrogen bonds were calculated using PLATON $^{37}$ and agree with the literature. ${ }^{37}$

\section{FT-IR spectra}

The FT-IR spectra of the complexes show a broad band near $3400 \mathrm{~cm}^{-1}$ assigned to $\mathrm{O}-\mathrm{H}$ stretching of the water molecules present. ${ }^{49}$ The new bands in the $3200-3300 \mathrm{~cm}^{-1}$ range can be assigned to $\mathrm{V}_{\mathrm{N}-\mathrm{H}}{ }^{48-50}$ These bands were not observed in the spectra of the free ligands, due to the presence of the broad $v_{\mathrm{O}-\mathrm{H}}$ band centered around $3400 \mathrm{~cm}^{-1}$. This shift in $v_{\mathrm{N}-\mathrm{H}}$ frequency confirms complexation to the $\mathrm{Cu}^{2+}$ center. Several weak bands observed in the $2850-3100 \mathrm{~cm}^{-1}$ range are attributed to aliphatic and aromatic $\mathrm{C}-\mathrm{H}$ groups. ${ }^{49}$ The strong carbonyl $v_{\mathrm{CO}}$ band around $1680 \mathrm{~cm}^{-1}$ and aromatic ring $v_{\mathrm{C}=\mathrm{C}}$ bands, around 1590 and $1470 \mathrm{~cm}^{-1}$ were not altered by complexation. ${ }^{48}$ The strong $v_{\mathrm{C}-\mathrm{O}}$ band around $1280 \mathrm{~cm}^{-1}$ was attributed to the naphthoquinonato group..$^{47,51}$

\section{EPR Spectra}

The EPR spectra of complexes 1-13 were measured in the solid state at liquid nitrogen temperature. The Hamiltonian parameters (Table 5) obtained in 


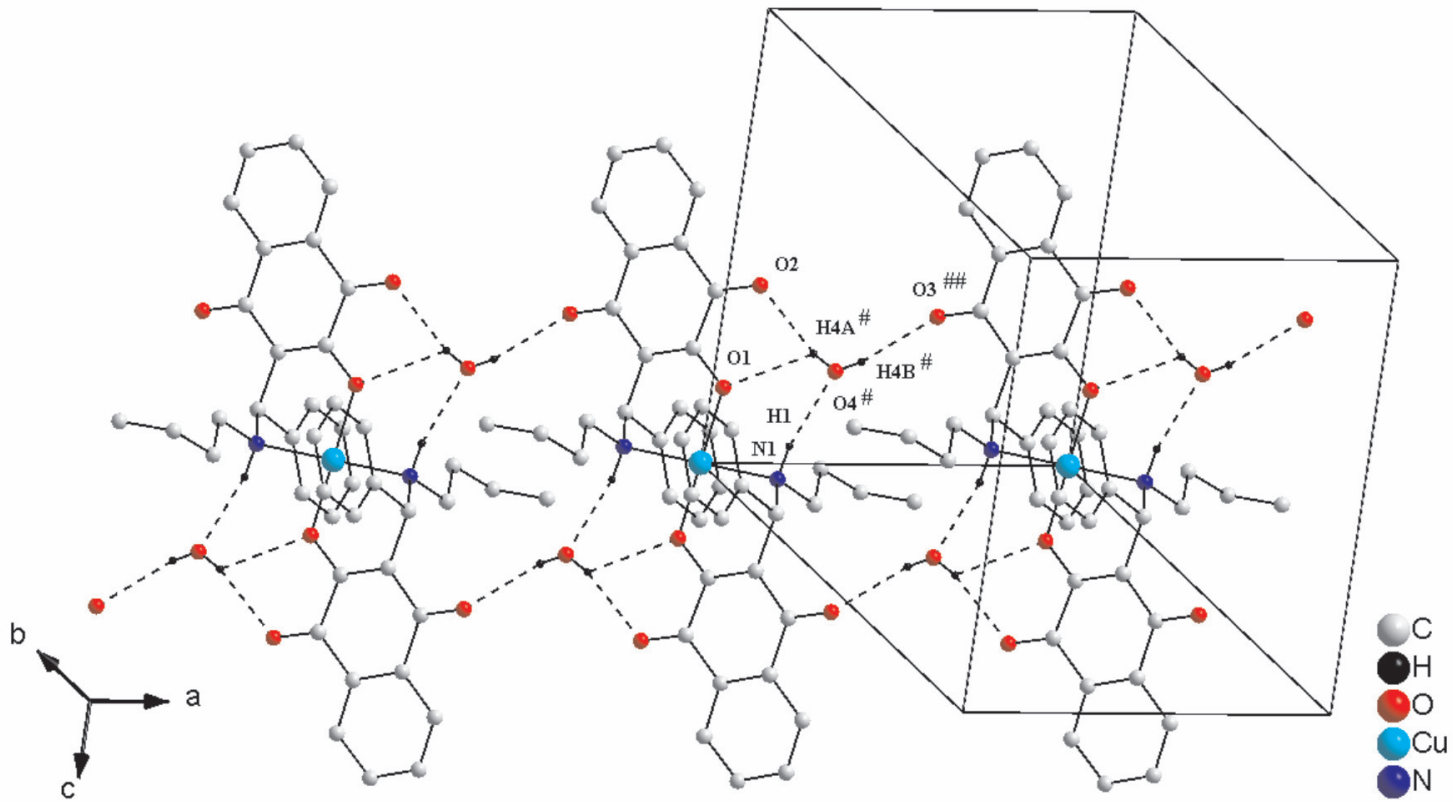

Figure 6. View of the self-assembly 1D by classical hydrogen bonds along [100] crystallography direction. (Symmetry code for 1: $(\#)=x, 1+y, z$; $(\# \#)=1+x, y, z)$.

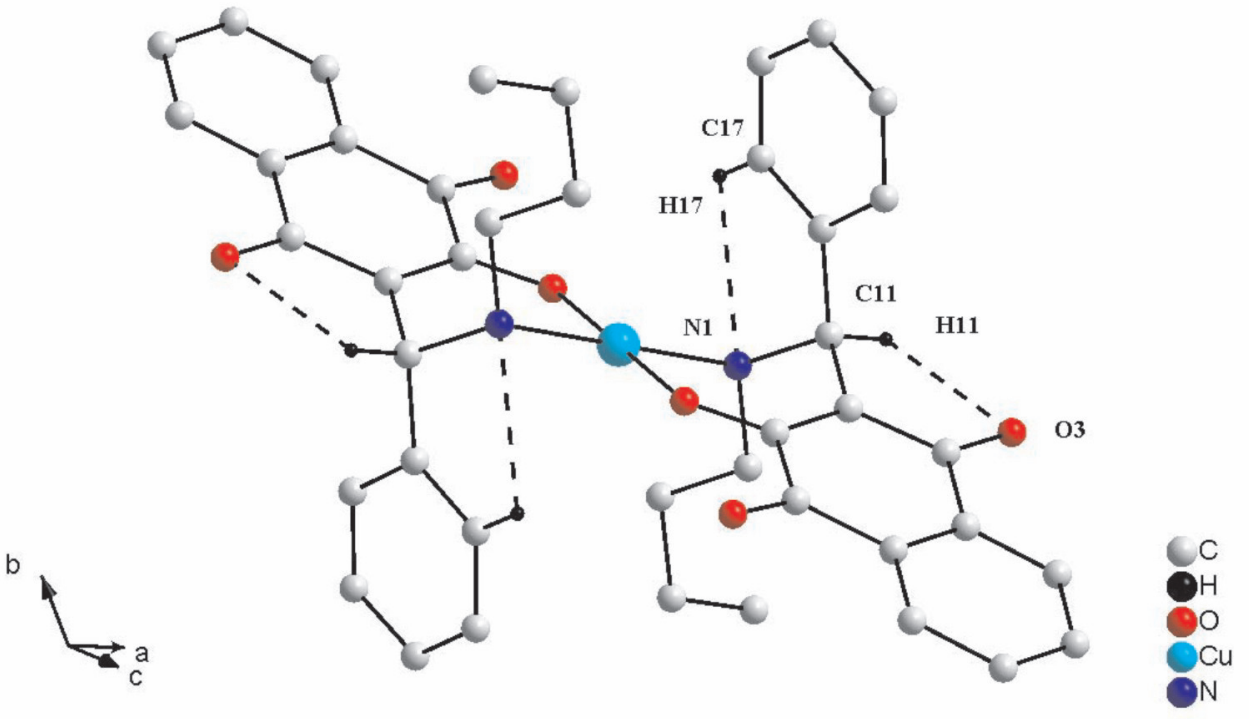

Figure 7. View of intramolecular interactions via non classical hydrogen bonds in complex $\mathbf{1}$.

Table 3. Geometric parameters for non-classical H bonds for complex 1 (Å, degree)

\begin{tabular}{lcccc}
\hline $\mathrm{D}-\mathrm{H} \cdots \mathrm{A}$ & $\mathrm{D}-\mathrm{H}$ & $\mathrm{H} \cdots \mathrm{A}$ & $\mathrm{D} \cdots \mathrm{A}$ & \multicolumn{1}{c}{$\mathrm{D}-\mathrm{H} \cdots \mathrm{A}$} \\
\hline $\mathrm{C}(17)-\mathrm{H}(17) \cdots \mathrm{N}(1)_{\text {intra }}$ & 0.93 & 2.60 & $2.926(5)$ & 101 \\
$\mathrm{C}(11)-\mathrm{H}(11) \cdots \mathrm{O}(3)_{\text {intra }}$ & $0.99(4)$ & $2.37(4)$ & $2.856(5)$ & $109(3)$ \\
$\mathrm{O}(4)^{\mathrm{a}}-\mathrm{H}(4 \mathrm{~A})^{\mathrm{a}} \cdots \mathrm{O}(1)$ & $0.76(7)$ & $2.56(7)$ & $3.079(5)$ & $126(5)$ \\
$\mathrm{O}(4)^{\mathrm{a}}-\mathrm{H}(4 \mathrm{~A})^{\mathrm{a}} \cdots \mathrm{O}(2)$ & $0.76(7)$ & $2.23(6)$ & $2.972(5)$ & $165(6)$ \\
$\mathrm{O}(4)^{\mathrm{a}}-\mathrm{H}(4 \mathrm{~B})^{\mathrm{a}} \cdots \mathrm{O}(3)^{\mathrm{b}}$ & $0.69(7)$ & $2.24(7)$ & $2.913(5)$ & $168(7)$ \\
$\mathrm{N}(1)-\mathrm{H}(1) \cdots \mathrm{O}(4)^{\mathrm{a}}$ & $0.88(4)$ & $2.16(4)$ & $3.018(5)$ & $167(4)$ \\
\hline
\end{tabular}

Symmetry code for $1:{ }^{(a)} \mathrm{x}, 1+\mathrm{y}, \mathrm{z} ;{ }^{(b)} 1+\mathrm{x}, \mathrm{y}, \mathrm{z}$. 


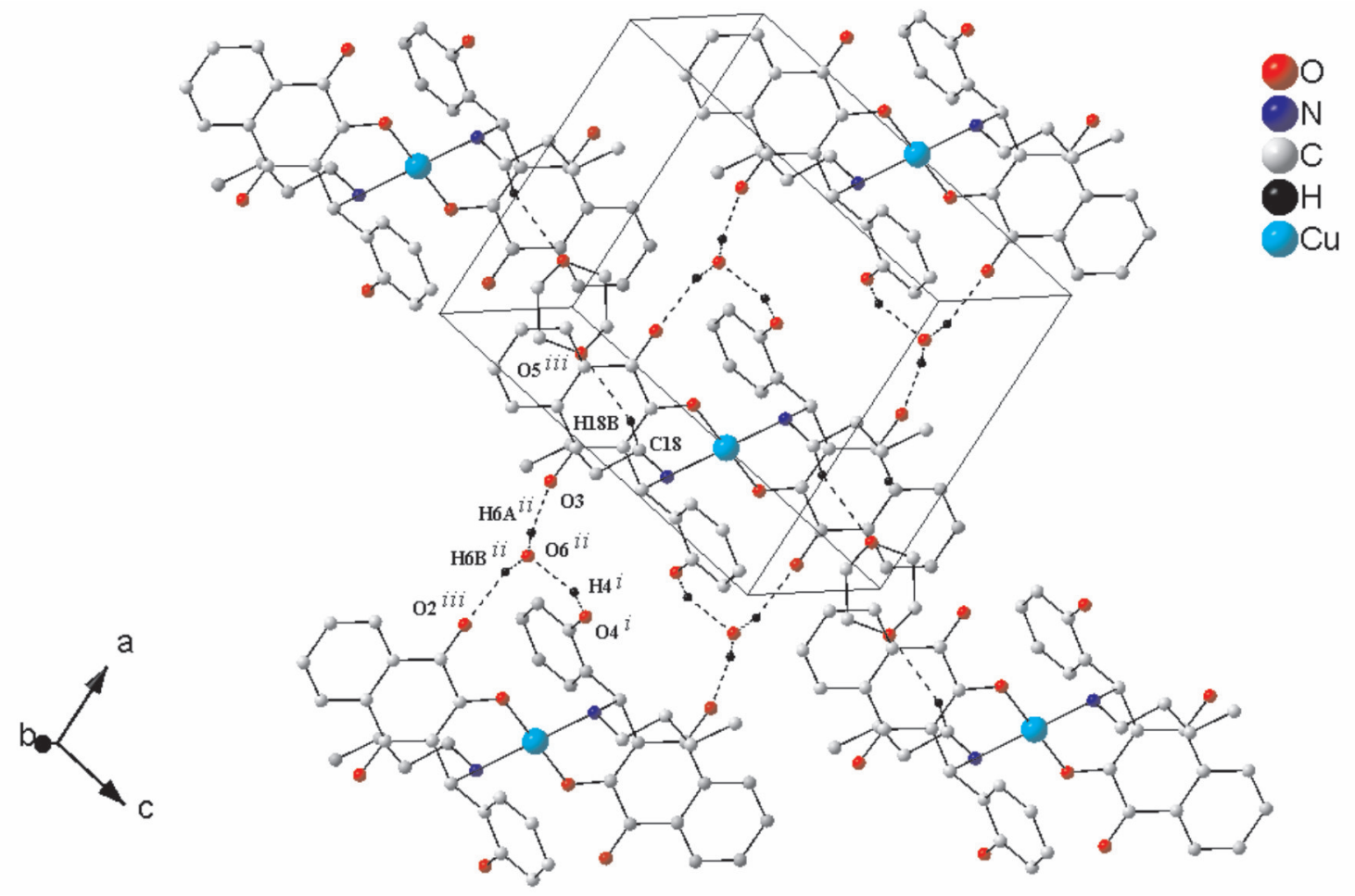

Figure 8. View of the 2D self-arrangement governed by water and dioxane molecules along [100] and [001] crystallography directions, respectively (symmetry code for 7 : $(i)=-1-x,-y, 1-z ;(i i)=-x, 1-y, 1-z ;(i i i)=-1+x, y, z)$.

b

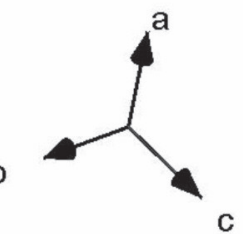

$\mathrm{c}$

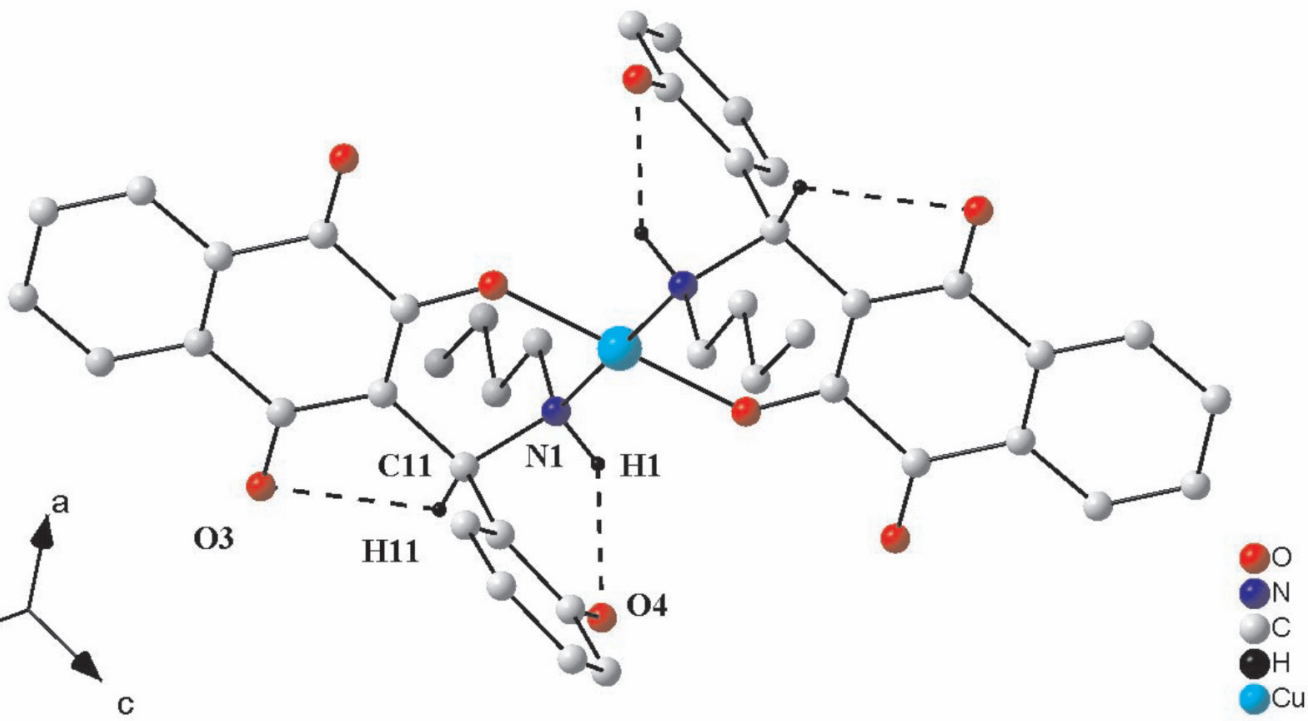

Figure 9. View of the intramolecular interactions in complex 7.

the simulations of the EPR spectra $g_{\|}>g_{\perp}>2$ and $A_{\|}=(188-200) \times 10^{-4} \mathrm{~cm}^{-1}$ are typical of elongated octahedral or square planar geometry, suggesting copper(II) sites with axial symmetry. ${ }^{52}$ All complexes, except for $\mathbf{2}$ and 12, show values of $\mathrm{g}_{\|} / \mathrm{A}_{\|}$between 110 and 122 thus confirming the square planar environment around the $\mathrm{Cu}^{2+}$ centre $^{53}$ as established for compounds $\mathbf{1}, \mathbf{2}$ and $\mathbf{7}$ by X-ray analysis. The experimental values, $g_{\|}>g_{\perp}$, also indicate that the unpaired electron is predominantly in the $\mathrm{dx}^{2}-\mathrm{y}^{2}$ orbital, which gives ${ }^{2} \mathrm{~B}_{1 \mathrm{~g}}$ as the ground state. The very low parallel and perpendicular components of the hyperfine coupling constant for complexes $\mathbf{2}$ and $\mathbf{1 2}$ (not resolved in the spectra) has been explained by considering a mixture of the $\mathrm{Cu}^{2+} \mathrm{dz}^{2}$ and $\mathrm{dx}^{2}-\mathrm{y}^{2}$ orbitals as the ground state. It is found that a $10 \%$ mixture of $\mathrm{dx}^{2}-\mathrm{y}^{2}$ and $\mathrm{dz}^{2}$ results in a $20 \%$ reduction in dipolar anisotropy..$^{54}$ 
Table 4. Geometric parameters for non-classical H bonds for complex 7 (, degree)

\begin{tabular}{lccc}
\hline$D-\mathrm{H} \cdots \mathrm{A}$ & $D-\mathrm{H}$ & $\mathrm{H} \cdots \mathrm{A}$ & \multicolumn{1}{c}{$D-\mathrm{H} \cdots \mathrm{A}$} \\
\hline $\mathrm{N}(1)-\mathrm{H}(1) \cdots \mathrm{O}(4)_{\text {intra }}$ & $0.93(4)$ & $2.08(4)$ & $2.818(3)$ \\
$\mathrm{C}(11)-\mathrm{H}(11) \cdots \mathrm{O}(3)_{\text {intra }}$ & 0.98 & 2.40 & $2.836(4)$ \\
$\mathrm{O}(4)^{i}-\mathrm{H}(4)^{i} \cdots \mathrm{O}(6)^{i i}$ & 0.82 & 1.95 & $136(3)$ \\
$\mathrm{O}(6)^{i i}-\mathrm{H}(6 \mathrm{~A})^{i i} \cdots \mathrm{O}(3)$ & 0.89 & 1.95 & 151 \\
$\mathrm{O}(6)^{i i}-\mathrm{H}(6 \mathrm{~B})^{i i} \cdots \mathrm{O}(2)^{i i i}$ & 0.86 & 2.03 & $2.833(3)$ \\
$\mathrm{C}(18)-\mathrm{H}(18 \mathrm{~B}) \cdots \mathrm{O}(5)^{i i i}$ & 0.97 & 2.53 & 169 \\
\hline
\end{tabular}

Symmetry code for $(7)$ : $(i)=-1-x,-y, 1-z ;(i i)=-x, 1-y, 1-z ;(i i i)=-1+x, y, z$.

Table 5. Spin-Hamiltonian parameters used in the simulated spectra of the copper(II) ion in complexes 1-13 in decreasing order of field strength

\begin{tabular}{lccccc}
\hline Complexes & $\mathrm{A}_{\perp}\left(\times 10^{-4} \mathrm{~cm}^{-1}\right)$ & $\mathrm{A}_{\|}\left(\times 10^{-4} \mathrm{~cm}^{-1}\right)$ & $\mathrm{g}_{\perp}$ & $\mathrm{g}_{\|}$ & $\mathrm{g}_{\|} / \mathrm{A}_{\|}$ \\
\hline $\mathbf{1}$ & 15 & 200 & 2.0500 & 2.2070 & 110 \\
$\mathbf{3}$ & 25 & 197 & 2.0350 & 2.2100 & 112 \\
$\mathbf{5}$ & 27 & 198 & 2.0350 & 2.2100 & 112 \\
$\mathbf{1 0}$ & 25 & 192 & 2.0470 & 2.2055 & 115 \\
$\mathbf{1 1}$ & 25 & 195 & 2.0470 & 2.2350 & 115 \\
$\mathbf{7}$ & 20 & 190 & 2.0550 & 2.2070 & 116 \\
$\mathbf{1 3}$ & 25 & 193 & 2.0440 & 2.2380 & 116 \\
$\mathbf{9}$ & 25 & 190 & 2.0450 & 2.2380 & 118 \\
$\mathbf{4}$ & 25 & 190 & 2.0450 & 2.2380 & 118 \\
$\mathbf{8}$ & 25 & 188 & 2.0480 & 2.2380 & 119 \\
$\mathbf{6}$ & 25 & 188 & 2.0488 & 2.2400 & -119 \\
$\mathbf{1 2}$ & ---- & 2.0630 & 2.1880 & - \\
\hline
\end{tabular}

The relatively low $\mathrm{g}_{\|}$values of the complexes are consistent with a $\mathrm{N}_{2} \mathrm{O}_{2}$ environment around the $\mathrm{Cu}^{2+}$ ions. ${ }^{52}$ As expected, the ligand field strength depends mainly on the nature of the $\mathrm{R}^{4}$ substituent on the nitrogen, the highest ligand field being observed for complexes of ligands containing $\mathrm{R}^{4}=$ butyl, independently of the nature of $\mathrm{R}^{2}$ and $R^{3}$. The presence of the hydroxyl group $\left(R^{1}=\mathrm{OH}\right)$, however, may lead to a decrease in the ligand field, as observed for complex 7, compared with complex 1 (Table 5), due to the presence of a $\mathrm{O}-\mathrm{H} . . . \mathrm{N} 1$ hydrogen bond that reduces the Lewis basicity of N1. This interaction is evidenced in the supramolecular arrangement of the structure of 7 . This interaction may be present in all complexes containing $\mathrm{R} 1=\mathrm{OH}(\mathbf{6}-13)$.

\section{$U V$-Vis spectra}

The electronic spectra of complexes 1-5 and 12, recorded in $\mathrm{CHCl}_{3}$ solution, are characterized by two intense absorptions observed in the 425-315 $\mathrm{nm}$ range that are presumably due to a charge-transfer band from the naphthquinonato moiety to the metal ion, and to ligand based transitions. ${ }^{55}$ The band around $290 \mathrm{~nm}$ was not altered by coordination as it corresponds to $\pi-\pi^{*}$ transitions of the naphthoquinone ring. ${ }^{48-50}$. The $\mathrm{d}$-d band appears between 530-550 nm and can be attributed to a ${ }^{2} \mathrm{~A}_{1 \mathrm{~g}} \leftarrow{ }^{2} \mathrm{~B}_{1 \mathrm{~g}}$ transition which supports the square planar geometry for the complexes in solution..$^{50}$

The spectra of complexes 6-11 and $\mathbf{1 3}$ were recorded in DMSO solution due to their low solubility in $\mathrm{CHCl}_{3}$. All exhibit two intense absorption bands: the band observed around $450 \mathrm{~nm}$ was assigned to charge-transfer from the naphthoquinonate moiety to the metal ion and that at $277 \mathrm{~nm}$, also present in the spectra of the respective free ligands, to $\pi-\pi^{*}$ transitions of the naphthoquinone ring. No d-d transition band was observed, which suggests coordination of DMSO molecules and distorted octahedral coordination environment of the $\mathrm{Cu}^{2+}$ ion. 


\section{Antibacterial activity}

The antibacterial activity of Mannich bases HL1HL13 and complexes 1-13 was evaluated against seven strains of bacteria: Bacillus cereus (BC), Bacillus subtilis (BS), Escherichia coli (EC), Enterococcus faecalis (EF), Klebsiella pneumoniae (KP), Pseudomonas aeruginosa (PA) and Staphylococcus aureus (SA). The results are reported in Table 6 where the MIC values are expressed in $\mu \mathrm{mol} \mathrm{L} \mathrm{L}^{-1}$. Cloramphenicol was used as a positive control in all tests. Compounds 2-hydroxy-1,4-naphthoquinone (A), 2-amino-3-hydroxy-1,4-naphthquinone (B) and lapachol (C) were also tested for comparison.

Two Mannich bases and four complexes exhibited similar or higher activity than Chloramphenicol against three strains of bacteria (B. subtilis, E. coli (EC) and
S. aureus), whereas the other compounds, including 2-hydroxy-1,4-naphthoquinone (A, Entry 14), 2-amino3-hydroxy-1,4-naphthquinone (B, Entry 15) and lapachol (C Entry 16) only inhibited bacterial growth above $200 \mu \mathrm{mol} \mathrm{L}{ }^{-1} ; \mathrm{CuCl}_{2} \cdot 2 \mathrm{H}_{2} \mathrm{O}$ only inhibit bacterial growth above $3000 \mu \mathrm{mol} \mathrm{L}-1$.

Mannich bases HL11 (Entry 20) and HL13 (Entry 13) strongly inhibited the growth of $E$. coli (at 20 and $40 \mu \mathrm{mol} \mathrm{L}{ }^{-1}$, i.e. 8 and $22 \mu \mathrm{g} \mathrm{mL} \mathrm{m}^{-1}$, respectively) and $S$. aureus (at 40 $\mu \mathrm{mol} \mathrm{L}{ }^{-1}$, i.e. 19 and $22 \mu \mathrm{g} \mathrm{mL} \mathrm{m}^{-1}$, respectively). As shown in Figure 1, these compounds contain a 2-hydroxyphenyl group $\left(\mathrm{R}^{1}=\mathrm{OH}\right)$ which is substituted at the 5-position with $\mathrm{R}^{3}=\mathrm{Me}\left(\right.$ HL11) or $\mathrm{Br}\left(\right.$ HL13), respectively, and $\mathrm{R}^{4}=\mathrm{Bn}$. Thus, the nature of the $\mathrm{R}^{3}$ substituent appears to be of lesser importance than that of the lateral chain $\mathrm{R}^{4}$, considering that compound HL10 (Entry 10) with $\mathrm{R}^{3}=\mathrm{Me}$ (similar to HL11)

Table 6. Antibacterial activity data obtained from the standard dilution techniques of Mannich bases HL1-HL13, complexes 1-13, 2-hydroxy-1,4naphthoquinone (A), 2-amino-3-hydroxy-1,4-naphthquinone (B) and lapachol (C), tested against the following strains of bacteria: Bacillus cereus (BC), Bacillus subtilis (BS), Escherichia coli $(\mathbf{E C})$, Enterococcus faecalis $(\mathbf{E F})$, Klebsiella pneumoniae $(\mathbf{K P})$, Pseudomonas aeruginosa $(\mathbf{P A})$ and Staphylococcus aureus (SA). Positive control: Chloramphenicol

\begin{tabular}{|c|c|c|c|c|c|c|c|c|}
\hline Compound & Entry & BC & BS & EC & EF & $\mathbf{K P}$ & PA & SA \\
\hline HL1 & 1 & $>200$ & $>200$ & $>200$ & $>200$ & $>200$ & $>200$ & $>200$ \\
\hline HL2 & 2 & $>200$ & $>200$ & $>200$ & $>200$ & $>200$ & $>200$ & $>200$ \\
\hline HL3 & 3 & $>200$ & $>200$ & $>200$ & $>200$ & $>200$ & $>200$ & $>200$ \\
\hline HL4 & 4 & $>200$ & $>200$ & $>200$ & $>200$ & 180 & $>200$ & $>200$ \\
\hline HL5 & 5 & $>200$ & $>200$ & 180 & $>200$ & $>200$ & $>200$ & $>200$ \\
\hline HL6 & 6 & $>200$ & $>200$ & 180 & $>200$ & $>200$ & $>200$ & $>200$ \\
\hline HL7 & 7 & $>200$ & $>200$ & $>200$ & $>200$ & $>200$ & $>200$ & $>200$ \\
\hline HL8 & 8 & $>200$ & 180 & 180 & $>200$ & $>200$ & $>200$ & 180 \\
\hline HL9 & 9 & 180 & $>200$ & 180 & 180 & $>200$ & $>200$ & 180 \\
\hline HL10 & 10 & $>200$ & $>200$ & $>200$ & $>200$ & $>200$ & $>200$ & $>200$ \\
\hline HL11 & 11 & $>200$ & 90 & 20 & $>200$ & $>200$ & $>200$ & 40 \\
\hline HL12 & 12 & 180 & 180 & $>200$ & $>200$ & $>200$ & 180 & 180 \\
\hline HL13 & 13 & $>200$ & 180 & 40 & $>200$ & $>200$ & $>200$ & 40 \\
\hline A & 14 & $>200$ & $>200$ & $>200$ & $>200$ & $>200$ & $>200$ & $>200$ \\
\hline B & 15 & $>200$ & $>200$ & $>200$ & $>200$ & $>200$ & $>200$ & $>200$ \\
\hline C & 16 & $>200$ & $>200$ & $>200$ & $>200$ & $>200$ & $>200$ & $>200$ \\
\hline Chloramphenicol & 17 & $40-90$ & $20-40$ & 90 & 90 & $40-90$ & 40 & $40-90$ \\
\hline 1 & 18 & $>200$ & $>200$ & $>200$ & $>200$ & $>200$ & $>200$ & $>200$ \\
\hline 2 & 19 & $>200$ & $>200$ & 180 & $>200$ & $>200$ & $>200$ & $>200$ \\
\hline 3 & 20 & $>200$ & $>200$ & $>200$ & $>200$ & $>200$ & $>200$ & $>200$ \\
\hline 4 & 21 & $>200$ & $>200$ & $>200$ & $>200$ & $>200$ & $>200$ & $>200$ \\
\hline 5 & 22 & $>200$ & $>200$ & 180 & $>200$ & $>200$ & $>200$ & $>200$ \\
\hline 6 & 23 & $>200$ & $>200$ & 90 & $>200$ & $>200$ & $>200$ & $>200$ \\
\hline 7 & 24 & $>200$ & $>200$ & $>200$ & $>200$ & $>200$ & $>200$ & $>200$ \\
\hline 8 & 25 & $>200$ & $>200$ & $>200$ & $>200$ & $>200$ & $>200$ & $>200$ \\
\hline 9 & 26 & 180 & $>200$ & 180 & $>200$ & $>200$ & $>200$ & $>200$ \\
\hline 10 & 27 & 180 & 180 & 90 & $>200$ & $>200$ & $>200$ & 180 \\
\hline 11 & 28 & 180 & 90 & 180 & 180 & 180 & 180 & 90 \\
\hline 12 & 29 & 180 & 90 & 90 & $>200$ & $>200$ & $>200$ & 90 \\
\hline 13 & 30 & $>200$ & 90 & 40 & $>200$ & $>200$ & $>200$ & 90 \\
\hline $\mathrm{CuCl}_{2} \cdot 2 \mathrm{H}_{2} \mathrm{O}$ & 31 & $>3000$ & $>3000$ & $>3000$ & $>3000$ & $>3000$ & $>3000$ & $>3000$ \\
\hline
\end{tabular}

Minimum inhibitory concentrations (MICs) are reported in $\mu \mathrm{mol} \mathrm{L}^{-1}$. 
and $\mathrm{R}^{4}=\mathrm{Bu}$ is much less active than $\mathrm{HL11}$ against all strains of bacteria. Solubility differences might be responsible for the changes in activity.

With a few exceptions, the complexes were less active than the respective pro-ligands, which is probably due to their lower solubility. Thus, the activity of HL11 (Entry 11) decreased upon complexation, from 20 to $90 \mu \mathrm{mol} \mathrm{L}^{-1}$ for 11 (E. coli) and from 20 to $90 \mu \mathrm{mol} \mathrm{L}^{-1}$ for $11(S$. aureus) (Entry 28), although slight increase in growth inhibition of all the other bacteria strains was observed (from $>200 \mu \mathrm{mol} \mathrm{L}^{-1}$ for HL11 to $180 \mu \mathrm{mol} \mathrm{L} \mathrm{L}^{-1}$ for 11).

Improvement of the activity of HL10 and H12 that only inhibited the growth of all strains of bacteria above 180-200 $\mu \mathrm{mol} \mathrm{L}^{-1}$ (Entries 10 and 12) was observed upon complexation: complex 10 (Entry 27) exhibits slight activity against $B$. cereus, B. subtilis and $S$. aureus $\left(180 \mu \mathrm{mol} \mathrm{L}^{-1}\right)$ and growth inhibition against $E$. coli $\left(90 \mu \mathrm{mol} \mathrm{L}^{-1}\right)$, and complex 12, against E. coli, E. faecalis and $S$. aureus above $90 \mu \mathrm{mol} \mathrm{L}^{-1}$ (Entry 29). Complexes $\mathbf{1 0}$ and $\mathbf{1 2}$ were formed from Mannich bases HL10 and HL12 that only differed from the very active ones, HL11 and HL13 with respect to the $\mathrm{R}^{4}$ group, butyl, instead of benzyl.

The effect of metal complexation on naphthoquinone antimicrobial agents has been discussed in the literature. ${ }^{56}$ Although metal chelation of the anion of 5-hydroxy-1,4naphthoquinone (juglone) has resulted in complexes with similar antibacterial effect ${ }^{57}$ or higher antibacterial activity, e.g. against Bacillus ssp and $S$. aureus, than juglone, complexation of the anions of a series of 5-amino-8hydroxy-1,4-naphthoquinones with $\mathrm{M}^{2+}(\mathrm{M}=\mathrm{Ni}, \mathrm{Co}, \mathrm{Fe}$, $\mathrm{Cu}$ and $\mathrm{Cr}$ ) resulted in reduced bacterial activity or lack of inhibition effect. ${ }^{55}$ Considering that redox active metals have been shown to be instrumental in naphthoquinone toxicity, ${ }^{11}$ the decreased activity observed in our work and by others is probably associated with decreased bioavailability of the aminonaphthoquinones as the result of decreased solubility upon complexation.

\section{Conclusions}

In conclusion, of the thirteen novel aminonaphthoquinones HL1-HL13 synthesized from lawsone, via the Mannich reaction, and their respective copper(II) complexes $\left[\mathrm{Cu}(\mathbf{L 1})_{2}\right]-\left[\mathrm{Cu}(\mathbf{L 1 3})_{2}\right]$, those containing a 2-hydroxyphenyl group $\left(\mathrm{R}^{1}=\mathrm{OH}\right)$ which is substituted at the 5-position, either with $\mathrm{R}^{3}=\mathrm{Me}$ or $\mathrm{Br}$, and with $\mathrm{R}^{4}=$ benzyl or butyl groups have strongly inhibited the growth especially of Escherichia coli and Staphylococcus aureus. In general complexes were found to be less active than the respective pro-ligands, probably due to their lower solubility. Further work is in progress to improve the bioavailability of the complexes of HL10-HL13.

\section{Supplementary Information}

Supplementary data are available free of charge at http:// jbcs.sbq.org.br, as PDF file.

Crystallographic data for the structural analysis of the three complexes have been deposited with the Cambridge Crystallographic Data Center, CCDC No. 703509 (1), 703510 (2) and 703511 (7). Copies of this information may be obtained free of charge from The Director, CCDC, 12 Union Road, Cambridge, CB2 1EZ, UK (fax: +44 1233336 033; e-mail: deposit@ccdc.cam.ac.uk).

\section{Acknowledgments}

We thank CNPq, CAPES, FINEP, PRONEX-FAPERJ and FAPERJ for financial support, and the $\mathrm{X}$-ray diffraction laboratory (LDRX) of Universidade Federal Fluminense for data collection.

\section{References}

1. Thompson, R. H.; Naturally Occurring Quinones IV: Recent Advances, Champman \& Hall: London, 1997.

2. Sacau, E. P.; Braun, A. E.; Ravelo, A. G.; Ferro, E. A.; Tokuda, H.; Mukainaka, T.; Nishino, H.; Bioorg. Med. Chem. 2003, 11, 483.

3. Chen, J.; Huang, Y.; Liu, G.; Afrasiabi, Z.; Sinn, E.; Padhye, S.; Ma. Y.; Toxicol. Appl. Pharmacol. 2004, 194, 40.

4. Ferreira, V. F.; Jorqueira, A.; Souza, A. M. T.; Silva, M. N.; de Souza, M. C. B. V.; Gouvêa, R. M.; Rodrigues, C. R.; Pinto, A. V.; Castro, H. C.; Santos, D. O.; Araújo, H. P.; Bourguignon, S. C.; Bioorg. Med. Chem. 2006, 14, 5459.

5. Santos, A. F.; Ferraz, P. A. L.; Pinto, A. V.; Pinto, M. C. F. R.; Goulart, M. O. F.; Sant'Ana, A. E. G.; Int. J. Parasitol. 2000, 30, 1199 .

6. Santos, E. V. M.; Carneiro, J. W. M.; Ferreira, V. F.; Bioorg. Med. Chem. 2004, 12, 87.

7. Kayser, O.; Kiderlen, A. F.; Laatsch, H.; Croft, S.; Acta Trop. 2000, 77, 307.

8. Gafner, S.; Wolfender, J. L.; Nianga, M.; Stoeckli-Evans, H.; Hostettman, K.; Phytochemistry 1996, 42, 1315.

9. Machado, T. B.; Pinto, A. V.; Pinto, M. C. F. R.; Leal, I. C. R.; Silva, M. G.; Amaral, A. C. F.; Kuster, R. M.; Netto-dosSantos, K. R.; Int. J. Antimicrob. Agents 2003, 21, 279.

10. Medina, L. F. C.; Hertz, P. F.; Stefani, V.; Henriques, J. A. P.; Zanotto-Filho, A.; Brandelli, A.; Biochem. Cell Biol. 2006, 84, 720 .

11. Zang, R.; Hirsh, O.; Mohsen, M.; Samuni, A.; Arch. Biochem. Biophys. 1994, 312, 385. 
12. Vargas, M. D.; Pinto, A. C.; Echevarria, A.; Esteves-Souza, A.; Camara, C. A.; Cunha, A. C.; Torres, J. C.; Lima, E. L. S.; J. Braz. Chem. Soc. 2006, 17, 439.

13. Esteves-Souza, A.; Figueiredo, D. V.; Esteves, A.; Câmara, C. A.; Vargas, M. D.; Pinto, A. C.; Echevarria, A.; Braz. J. Med. Biol. Res. 2007, 30, 1399.

14. Cunha, A. S.; Vargas, M. D.; Gattass, C. R.; Pinto, A. C.; Camara, C. A.; Esteves, A. S.; Lima, E. L. S.; Oncol. Rep. 2008, $20,225$.

15. Silva, M. S.; Camara, C. A.; Barbosa, T. P.; Soares, A. Z.; Cunha, L. C.; Pinto, A. C.; Vargas, M. D.; Bioorg. Med. Chem. 2005, 13, 193.

16. Barbosa, T. P.; Camara, C. A.; Silva, T. M. S.; Martins, R. M.; Pinto, A. C.; Vargas, M. D.; Bioorg. Med. Chem. 2005, 13, 6464.

17. Camara, C. A.; Silva, T. M. S.; Silva, T. G.; Barbosa, T. P.; Martins, R. M.; Vargas, M. D.; Pinto, A. C.; An. Acad. Bras. Ciênc. 2008, 80, 329.

18. Tandon, V. K.; Yadav, D. B.; Singh, R. V.; Chaturvedi, A. K.; Shukla, P. K.; Bioorg. Med. Chem. Lett. 2005, 15, 5324.

19. Tandon, V. K.; Yadav, D. B.; Chaturvedi, A. K.; Shukla, P. K.; Bioorg. Med. Chem. Lett. 2005, 15, 3288.

20. Riffel, A.; Medina, L. F.; Stefani, V.; Santos, R. C.; Bizani, D.; Brandelli, A.; Braz. J. Med. Biol. Res. 2002, 35, 811.

21. Anacona, J. R.; Moreno, A.; Main Group Met. Chem. 1999, 22 , 573.

22. Tandon, V. K.; Vaish, M.; Khanna, J. M.; Anand, N.; Arch. Pharmazie 1990, 323, 383.

23. Leffer, M. T.; Hathaway, R. J.; J. Am. Chem. Soc. 1948, 70, 3222.

24. Baramee, A.; Coppin, A.; Mortuaire, M.; Pelinski, L.; Tomavo, S.; Brocard, J.; Bioorg. Med. Chem. 2006, 14, 1294.

25. Santos, A. F.; Ferraz, P. A. L.; Pinto, A. V.; Pinto, M. C. F. R.; Goulart, M. O. F.; Sant'Ana, A. E. G.; Int. J. Parasitol. 2000, 30, 1199.

26. Zang, O.; Hirsh, M.; Mohsen, A.; Samuni; Arch. Biochem. Biophys. 1994, 312, 385.

27. Gokhale, N. H.; Shirisha, K.; Padhye, S. B.; Croft, S. L.; Kendrick, H. D.; Mckee, V.; Bioorg. Med. Chem. Lett. 2006, 16,430 .

28. Gokhale, N. H.; Shirisha, K.; Padhye, S. B.; Croft, S. L.; Kendrick, H. D.; Davies, W.; Anson, C. E.; Powell, A. K.; J. Inorg. Biochem. 2003, 95, 249.

29. Chen, J.; Huang, Y.; Liu, G.; Afrasiabi, Z.; Sinn, E.; Padhye, S.; Ma, Y.; Toxicol. Appl. Pharmacol. 2004, 197, 40.

30. Dalgliesh, C.E.; J. Am. Chem. Soc. 1949, 71, 1697.

31. Nonius; COLLECT, Nonius BV: Delft, The Netherlands, 1998.

32. Duisenberg, A. J. M.; Hooft, R. W. W.; Schreurs, A. M. M.; Kroon, J.; J. Appl. Crystallogr. 2000, 33, 893.

33. Duisenberg, A. J. M.; J. Appl. Crystallogr. 1992, 25, 92.
34. Sheldrick, G. M.; SADABS; Program for Empirical Absorption Correction of Area Detector Data, University of Göttingen: Germany, 1996.

35. Sheldrick, G. M.; SHELXS97; Program for Crystal Structure Solution, University of Göttingen: Germany, 1997.

36. Sheldrick, G. M.; SHELXL97; Program for Crystal Structure Refinement, University of Göttingen: Germany, 1997.

37. Spek, A. L.; J. Appl. Crystallogr. 2003, 36, 7.

38. Farrugia, L. J.; J. Appl. Crystallogr. 1999, 32, 837.

39. Farrugia, L. J.; J. Appl. Crystallogr. 1997, 30, 565.

40. National Committee for Clinical Laboratory Standards Methods for Dilution Antimicrobial Susceptibility Tests for Bacteria that Grow Aerobically, $6^{\text {th }}$ ed.; Approved Standard NCCLS Document M7-A6: Wayne, PA, 2003.

41. Langfield, R. D.; Scarano, F. J.; Heitzman, M. E.; Kondo, M.; Hammond, G. B.; Neto, C. C.; J. Ethnopharmacol. 2004, 94, 279.

42. Eloff, J. N.; Planta Med. 1998, 65, 711.

43. Addison, A. W. In Copper Coordination Chemistry: Biochemical and Inorganic Perspectives; Karlin, K. D.; Zubieta, J., eds., Academic Press: New York, 1983.

44. Muppidi, V. K.; Das, S.; Raghavaiah, P.; Pal, S.; Inorg. Chem. Commun. 2007, 10, 234.

45. Xie, Y.; Ni, J.; Liu, X.; Liu, Q.; Xu, X.; Transition Met. Chem. 2003, 28, 367.

46. Thakuria, H.; Das, G.; Polyhedron 2007, 26, 149.

47. Xie, Y.; Bu, W.; Chan, A. S. -C.; Xu, X.; Liu, Q.; Zhang, Z.; Yu, J.; Fan, Y.; Inorg. Chim. Acta 2000, 310, 257.

48. Muppidi, V. K.; Zacharias, P. S.; Pal, S.; J. Solid State Chem. 2007, 180, 132.

49. Muppidi, V. K.; Das, S.; Raghavaiah, P.; Pal, S.; Inorg. Chem. Commun. 2007, 10, 234.

50. Thakuria, H.; Das, G.; Polyhedron 2007, 26, 149.

51. Xie, Y.; Bu, W.; Chan, A. S. -C.; Xu, X.; Liu, Q.; Zhang, Z.; Yu, J.; Fan, Y.; Inorg. Chim. Acta 2000, 310, 257.

52. Neves, A.; Rossi, L. M.; Bortoluzzi, A. J.; Mangrich, A. S.; Haase, W.; Werner, R.; J. Braz. Chem. Soc. 2001, 12, 747.

53. Sakaguchi, U.; Addison, A. W.; J. Chem. Soc., Dalton Trans. 1979, 600.

54. Mendes, I. C.; Moreira, J. P.; Speziali, N. L.; Mangrich, A. S.; Takahashi, J. A.; Beraldo, H.; J. Braz. Chem. Soc. 2006, 17, 1571.

55. Ma, S.; Zhu, W.; Xu, M.; Wang, Y.; Guo, Q.; Liu, Y.; Polyhedron 2003, 22, 3249.

56. Brandelli, A.; Bizani, D.; Martinelli, M.; Stefani, V.; Gerbase, A. E.; Braz. J. Pharm. Sci. 2004, 40, 247.

57. Joshi, C. R.; Jagtap, G. S.; Chalgen, S. V.; Indian J. Pharmaceut. Sci. 1988, 50, 107.

58. Bakolachristianopoulou, M. N.; Ecateriniadou, L. B.; Sarris, K. J.; Eur. J. Med. Chem. 1986, 21, 385.

Received: March 3, 2009

Web Release Date: April 24, 2009 


\section{Novel Aminonaphthoquinone Mannich Bases Derived from Lawsone and their Copper (II) Complexes: Synthesis, Characterization and Antibacterial Activity}

\section{Amanda P. Neves, ${ }^{a}$ Cláudia C. Barbosa, ${ }^{a}$ Sandro J. Greco, ${ }^{a, \#}$ Maria D. Vargas, ${ }^{a, *}$ Lorenzo C. Visentin, ${ }^{b}$ Carlos B. Pinheiro, ${ }^{c}$ Antônio S. Mangrich, ${ }^{d}$ Jussara P. Barbosa ${ }^{e}$ and Gisela L. da Costa ${ }^{e}$}

aInstituto de Química, Universidade Federal Fluminense, Campus do Valonguinho, Centro, 24020-150 Niterói-RJ, Brazil

${ }^{b}$ Instituto de Química, Universidade Federal do Rio de Janeiro, Ilha do Fundão, 21945-970 Rio de Janeiro-RJ, Brazil

${ }^{c}$ Departamento de Física, Universidade Federal de Minas Gerais, Av. Antônio Carlos, 6627, Pampulha, 31270-901 Belo Horizonte-MG, Brazil

${ }^{d}$ Departamento de Química, Centro Politécnico, Universidade Federal do Paraná, 81531-970 Curitiba-PR, Brazil eInstituto Oswaldo Cruz, CP 926, 21045-900 Rio de Janeiro-RJ, Brazil

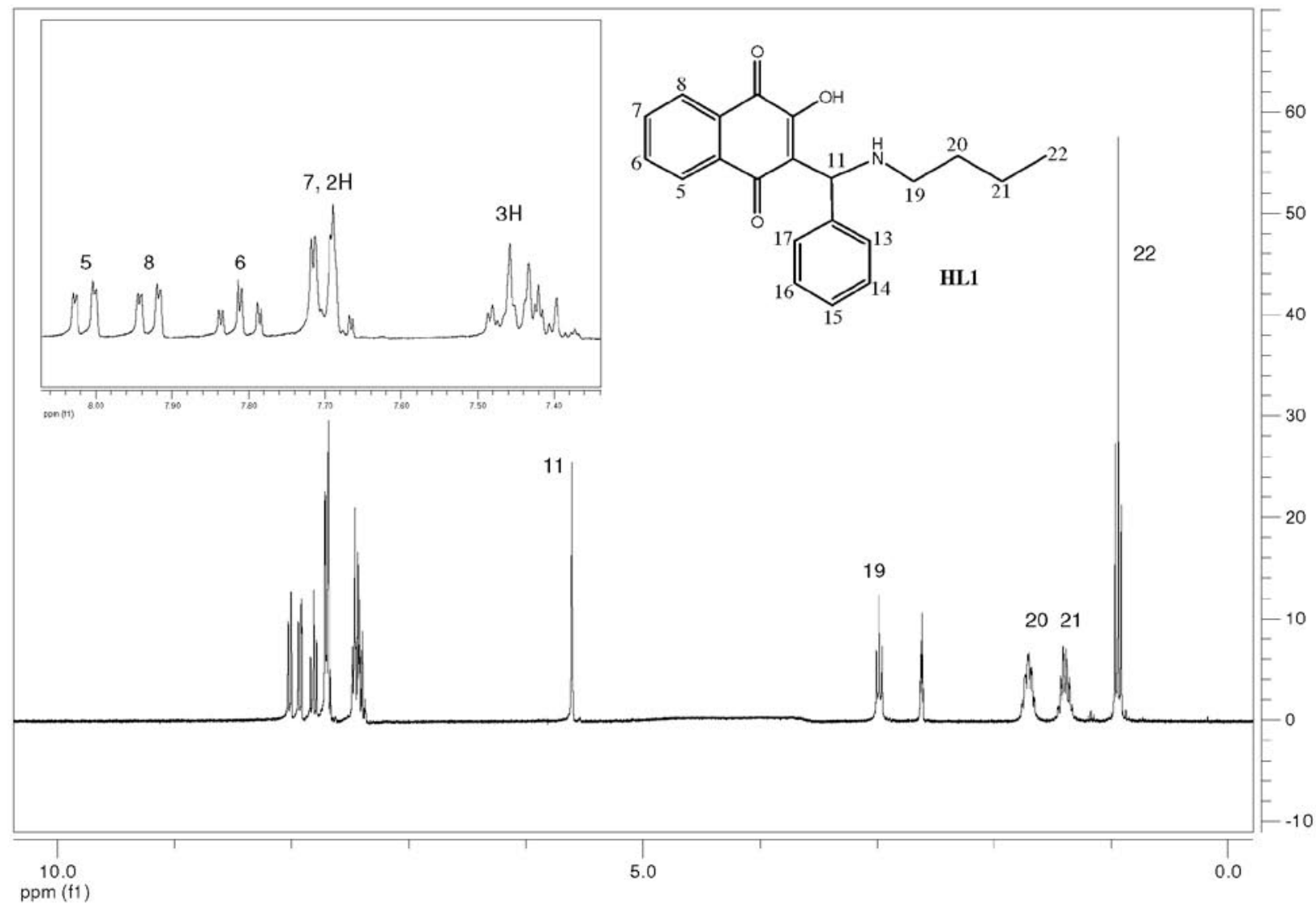

Figure S1. ${ }^{1} \mathrm{H}$ NMR spectrum of HL1.

*e-mail: mdvargas@vm.uff.br

\# Present Address: Universidade Federal do Espírito Santo, Centro Universitário Norte do Espírito Santo, Rua Humberto de Almeida Franklin, 257, Universitário, 29933-480 São Mateus-ES, Brazil 


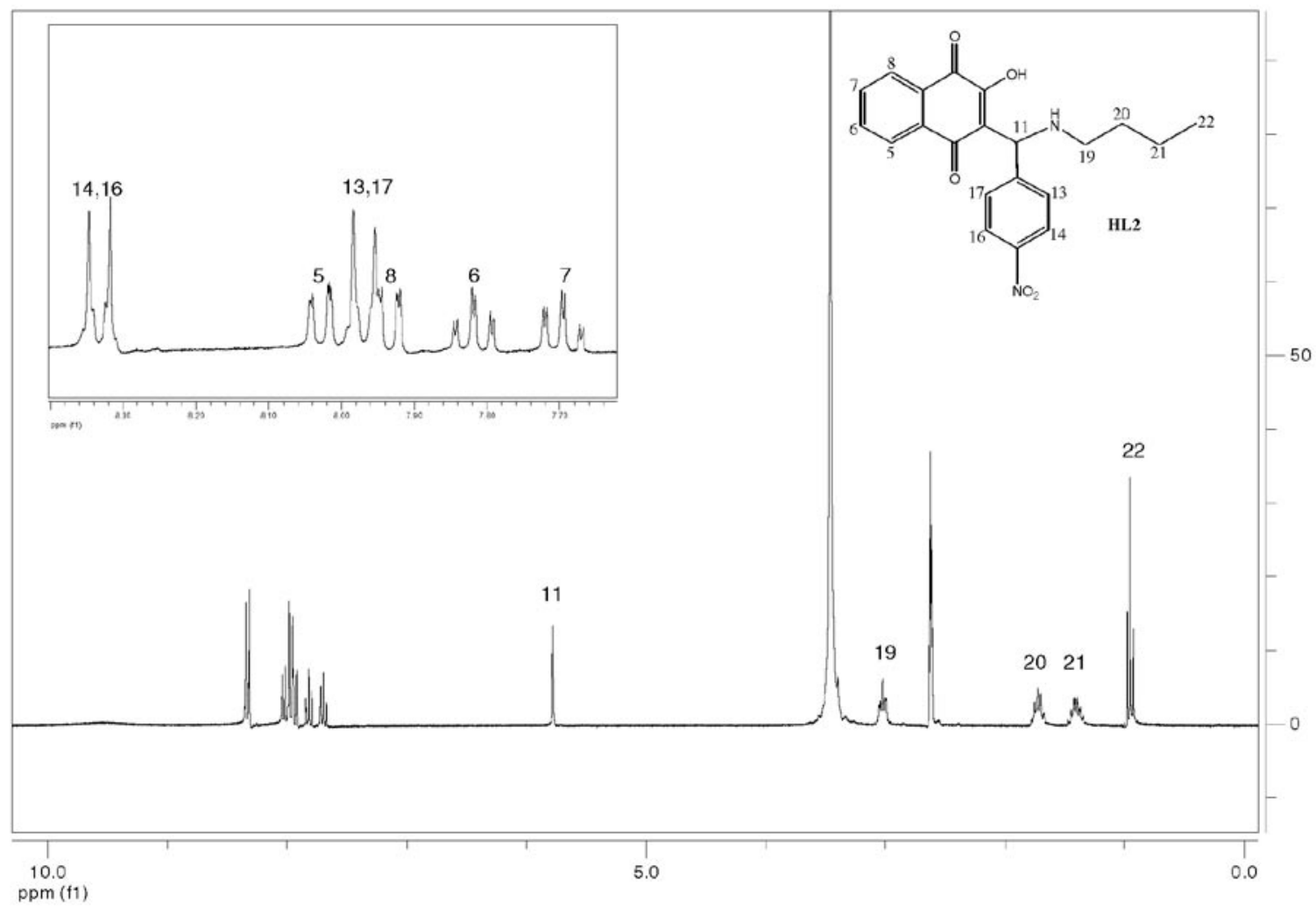

Figure S2. 'H NMR spectrum of HL2.

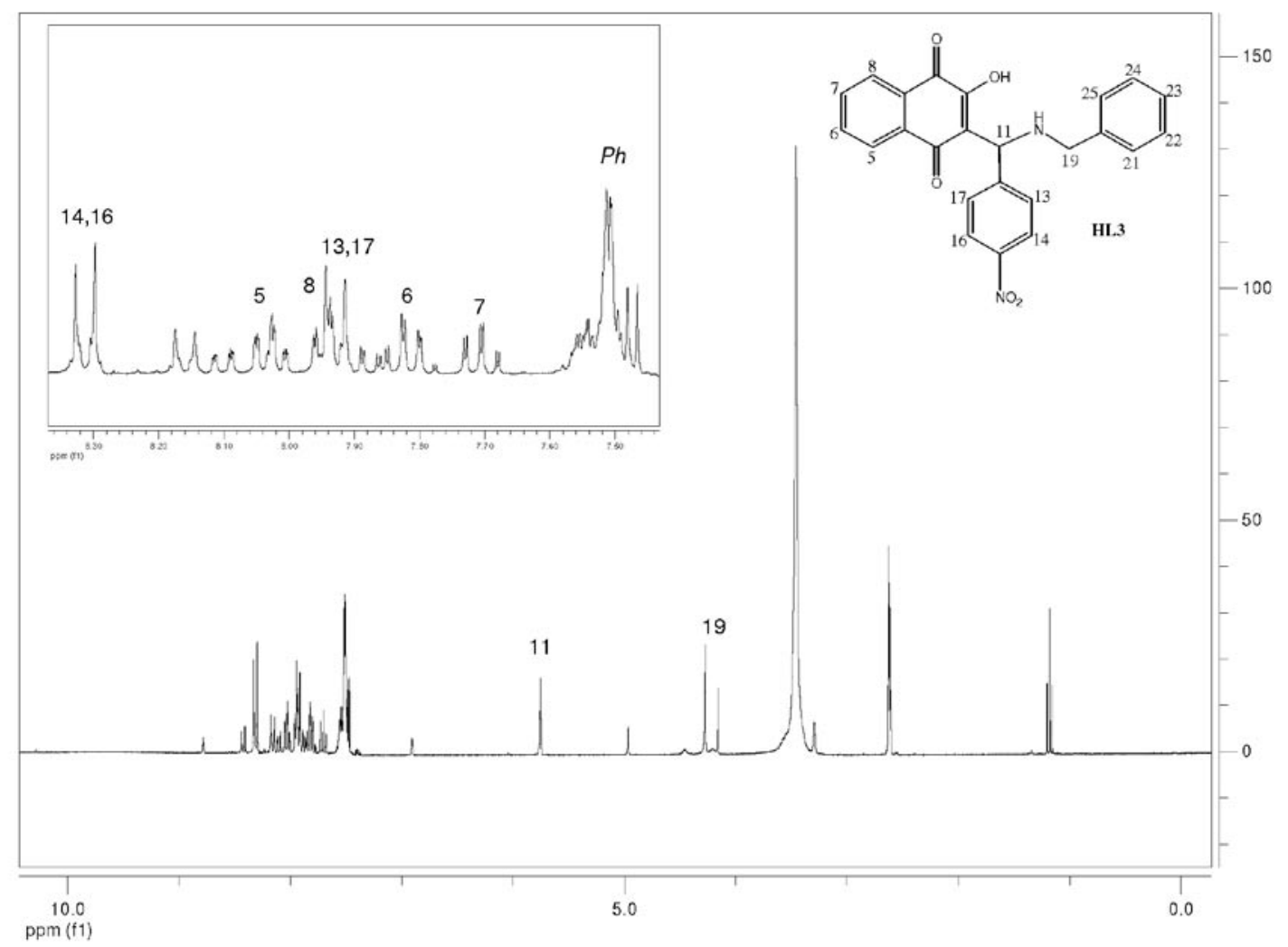

Figure S3. ${ }^{1} \mathrm{H}$ NMR spectrum of HL3. 


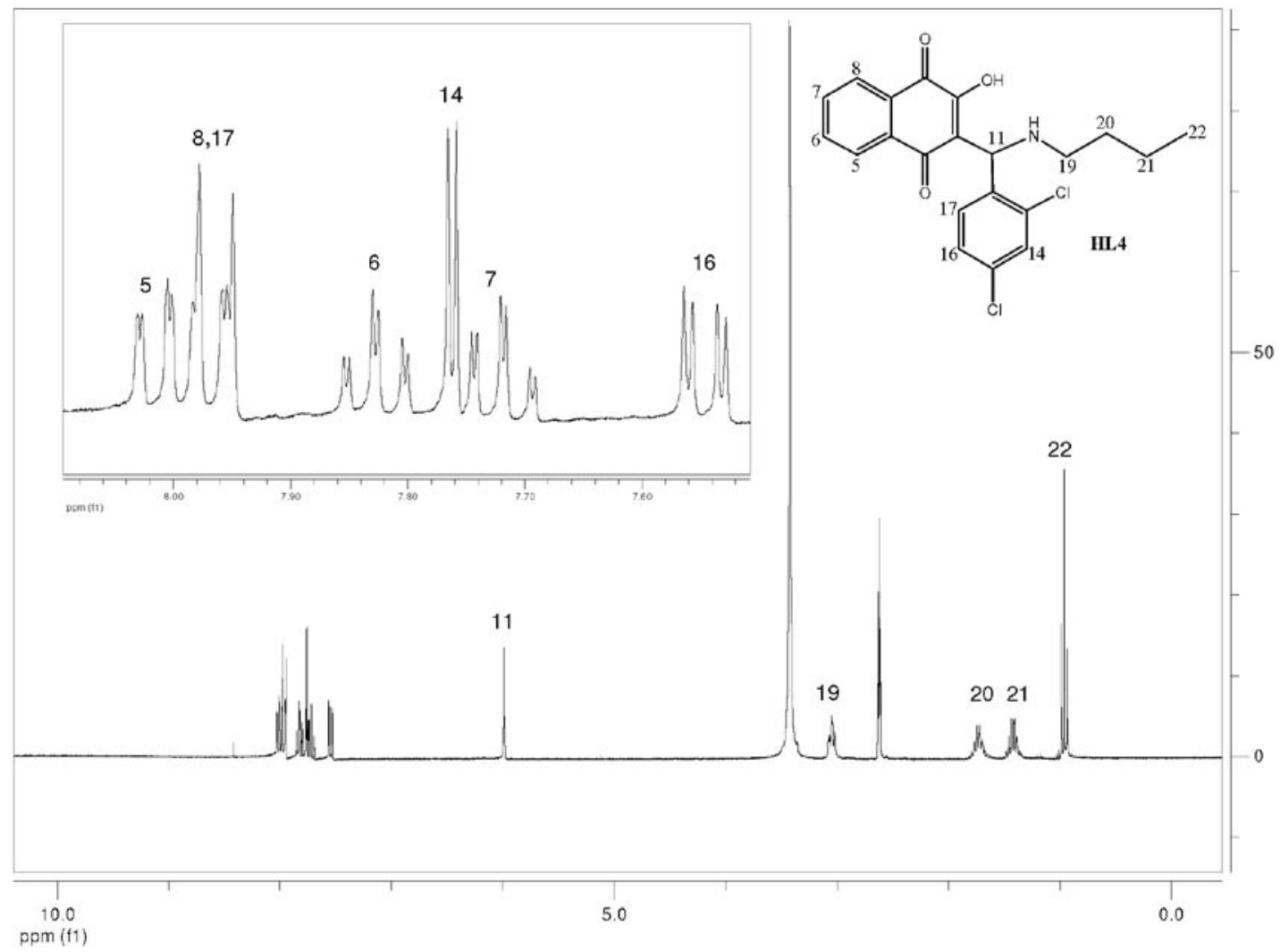

Figure S4. ${ }^{1} \mathrm{H}$ NMR spectrum of HL4.

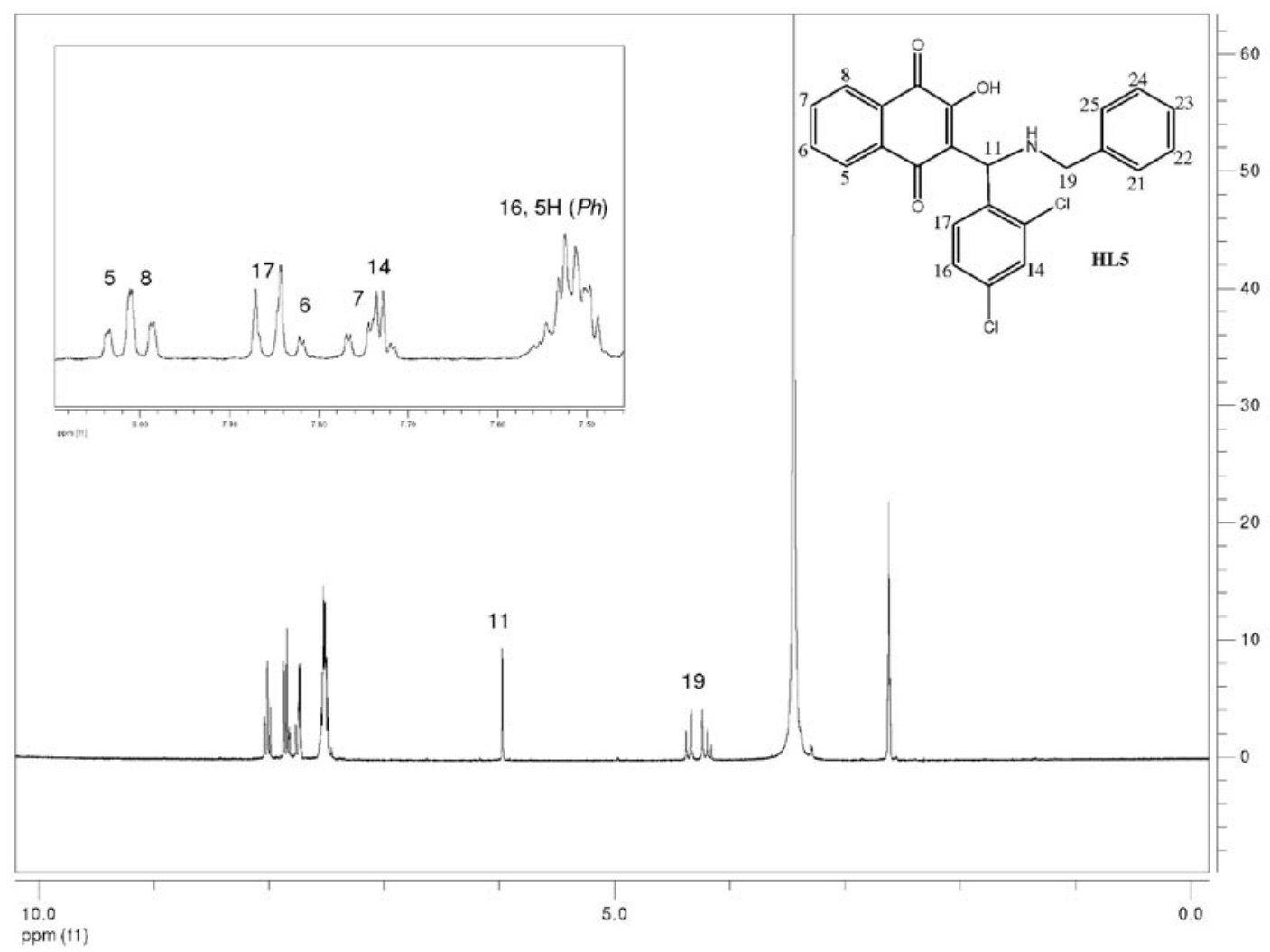

Figure S5. ${ }^{1} \mathrm{H}$ NMR spectrum of HL5. 


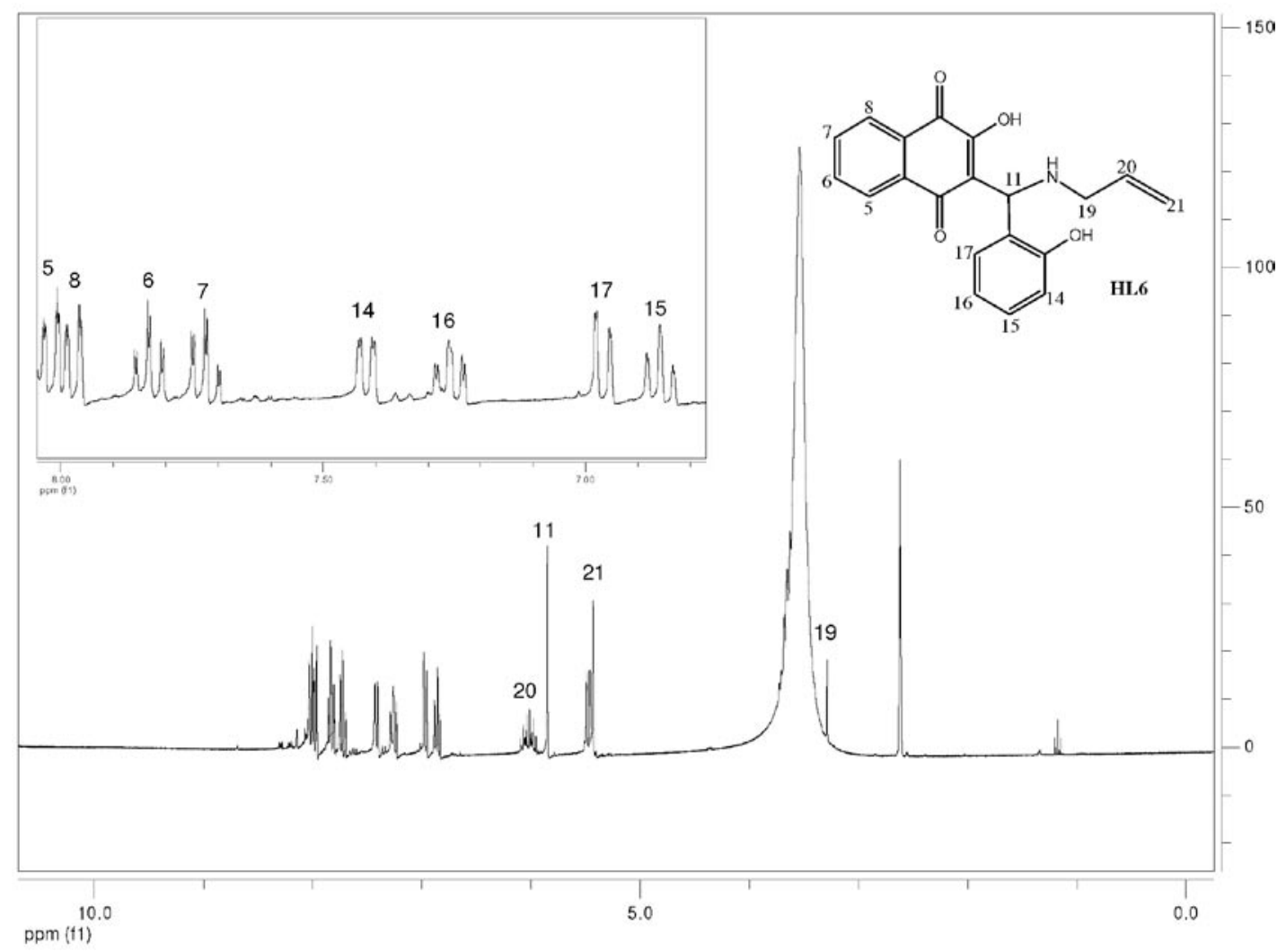

Figure S6. ' H NMR spectrum of HL6.

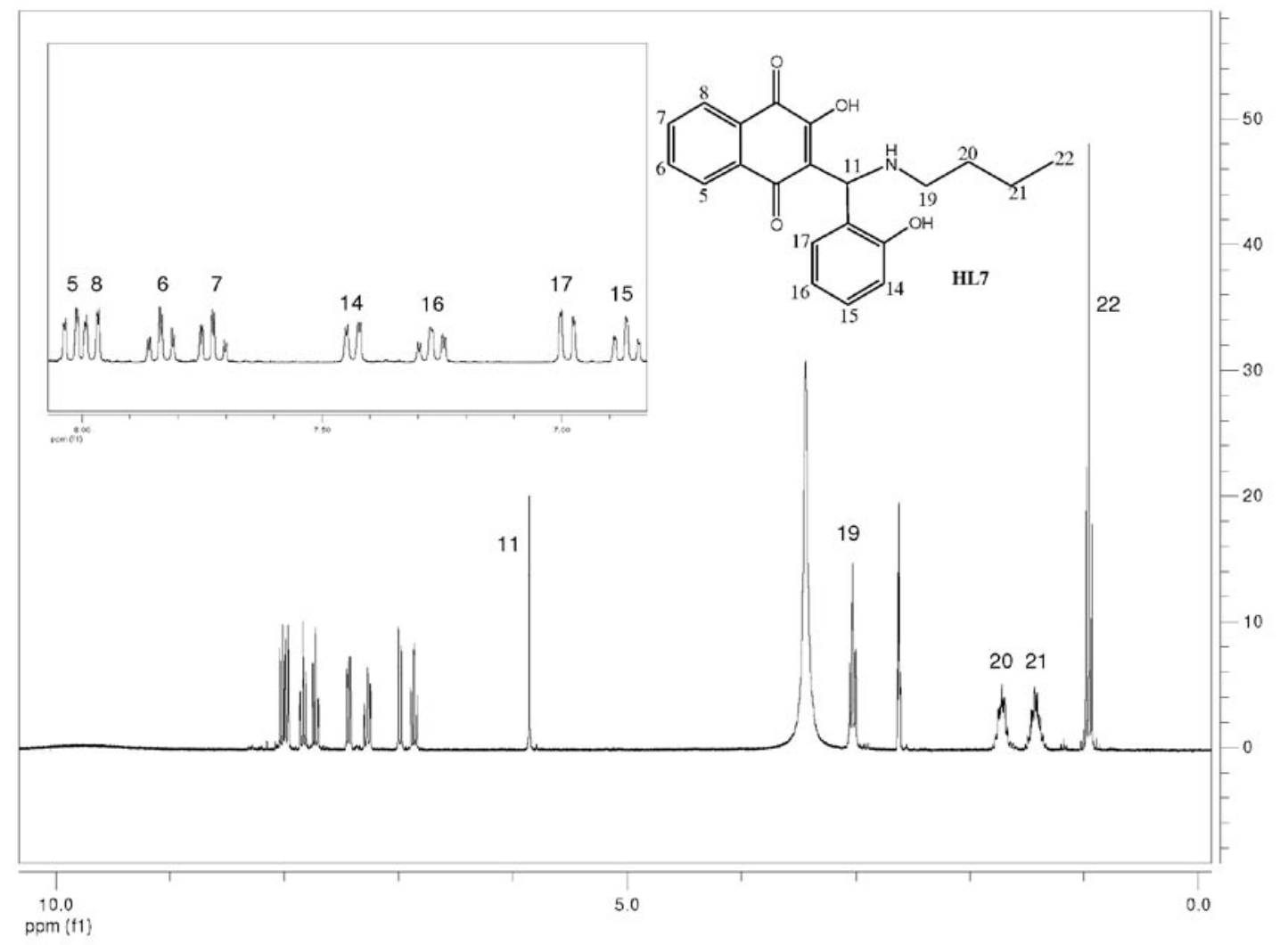

Figure S7. ${ }^{1} \mathrm{H}$ NMR spectrum of HL7. 


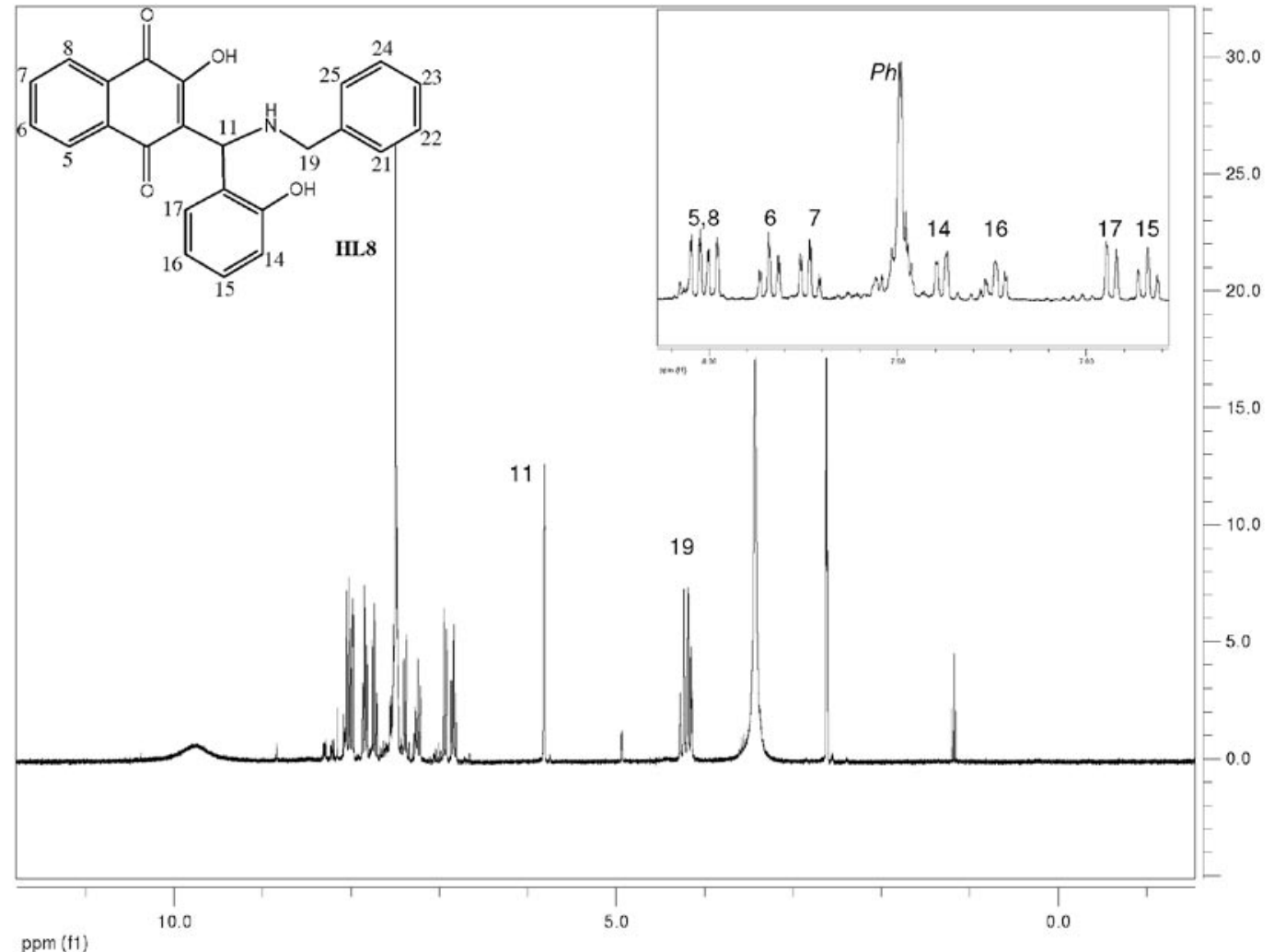

Figure S8. ${ }^{1} \mathrm{H}$ NMR spectrum of HL8.

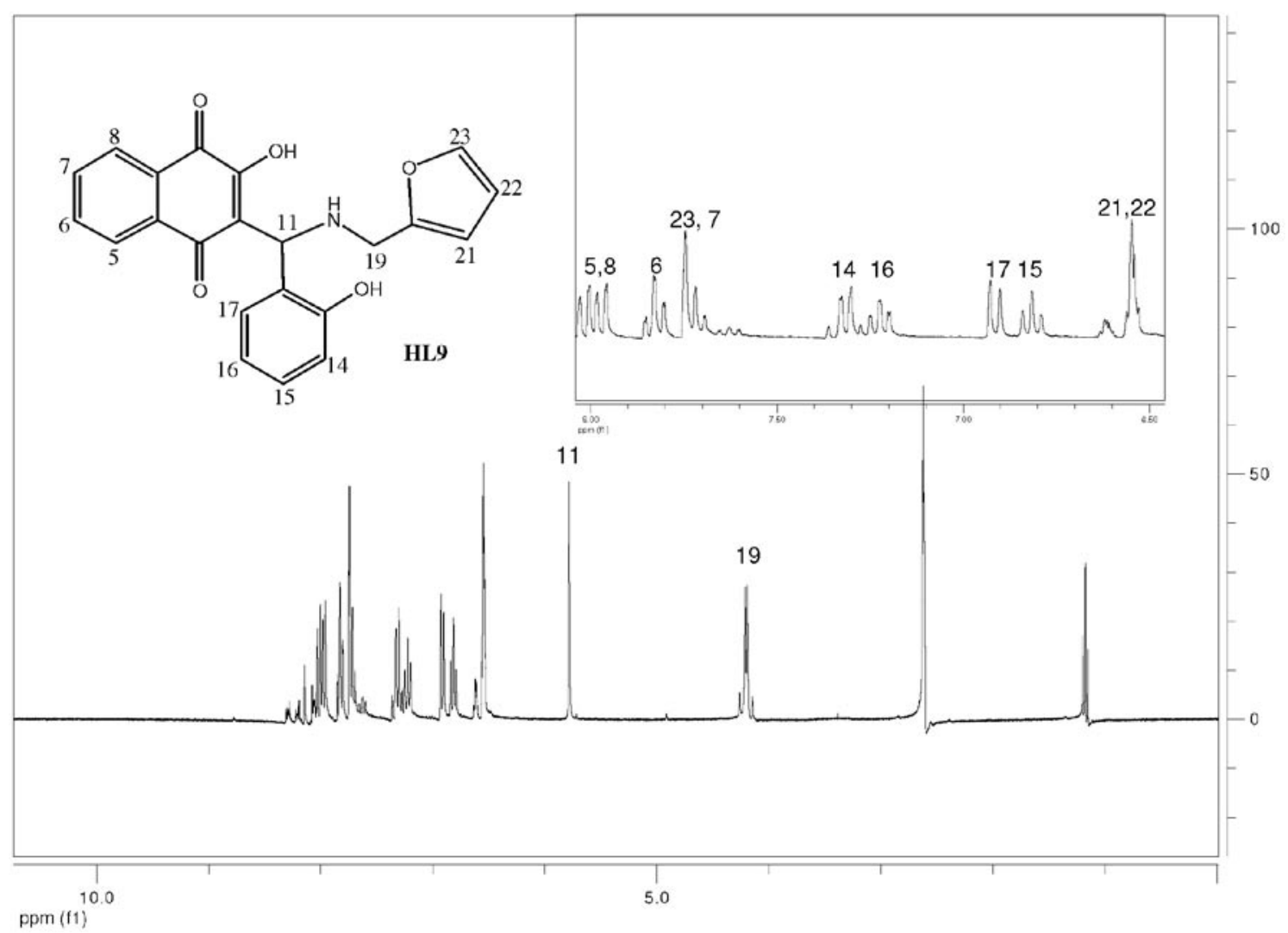

Figure S9. ${ }^{1} \mathrm{H}$ NMR spectrum of HL9. 


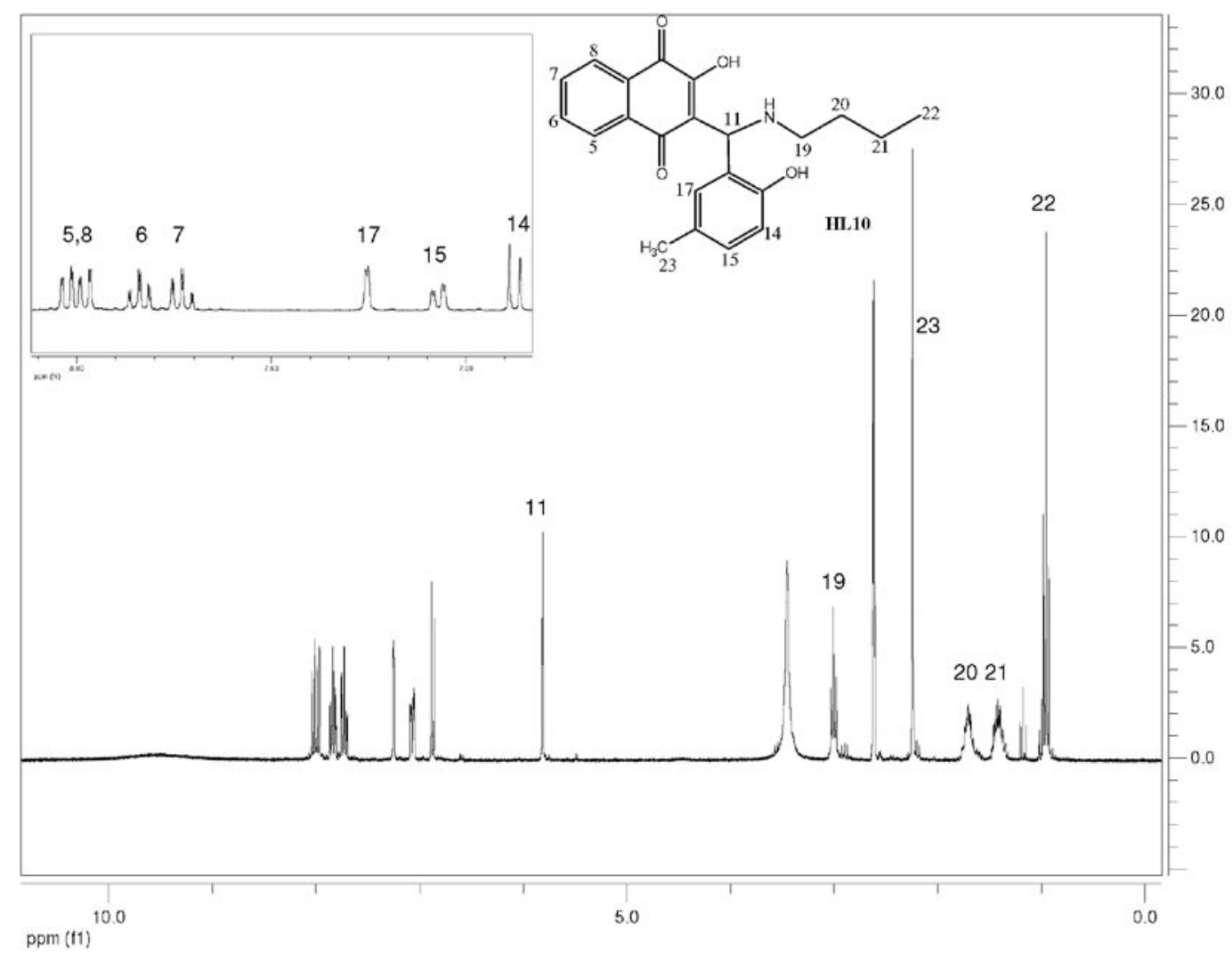

Figure S10. ${ }^{1} \mathrm{H}$ NMR spectrum of HL10.

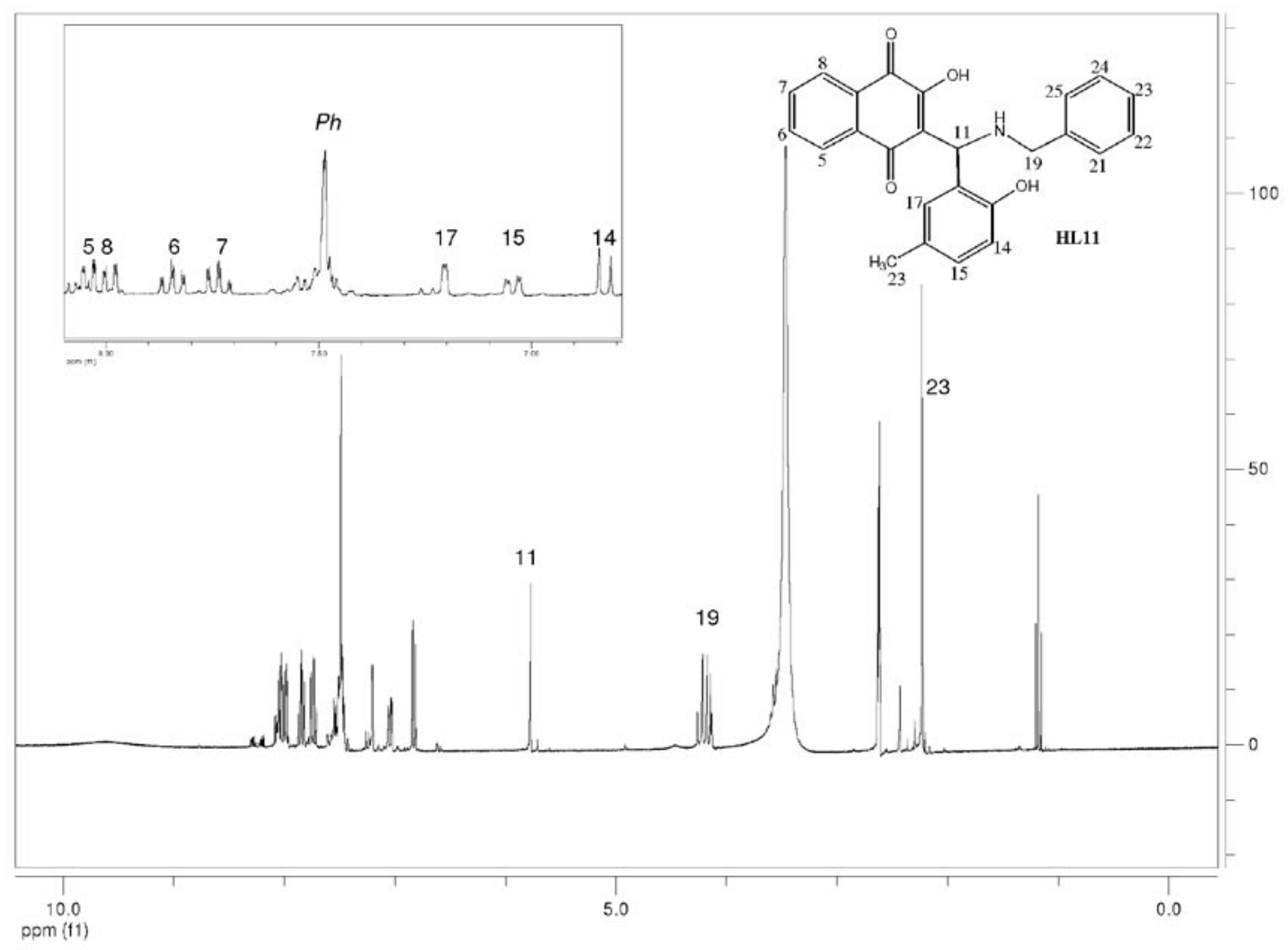

Figure S11. ${ }^{1} \mathrm{H}$ NMR spectrum of HL11. 


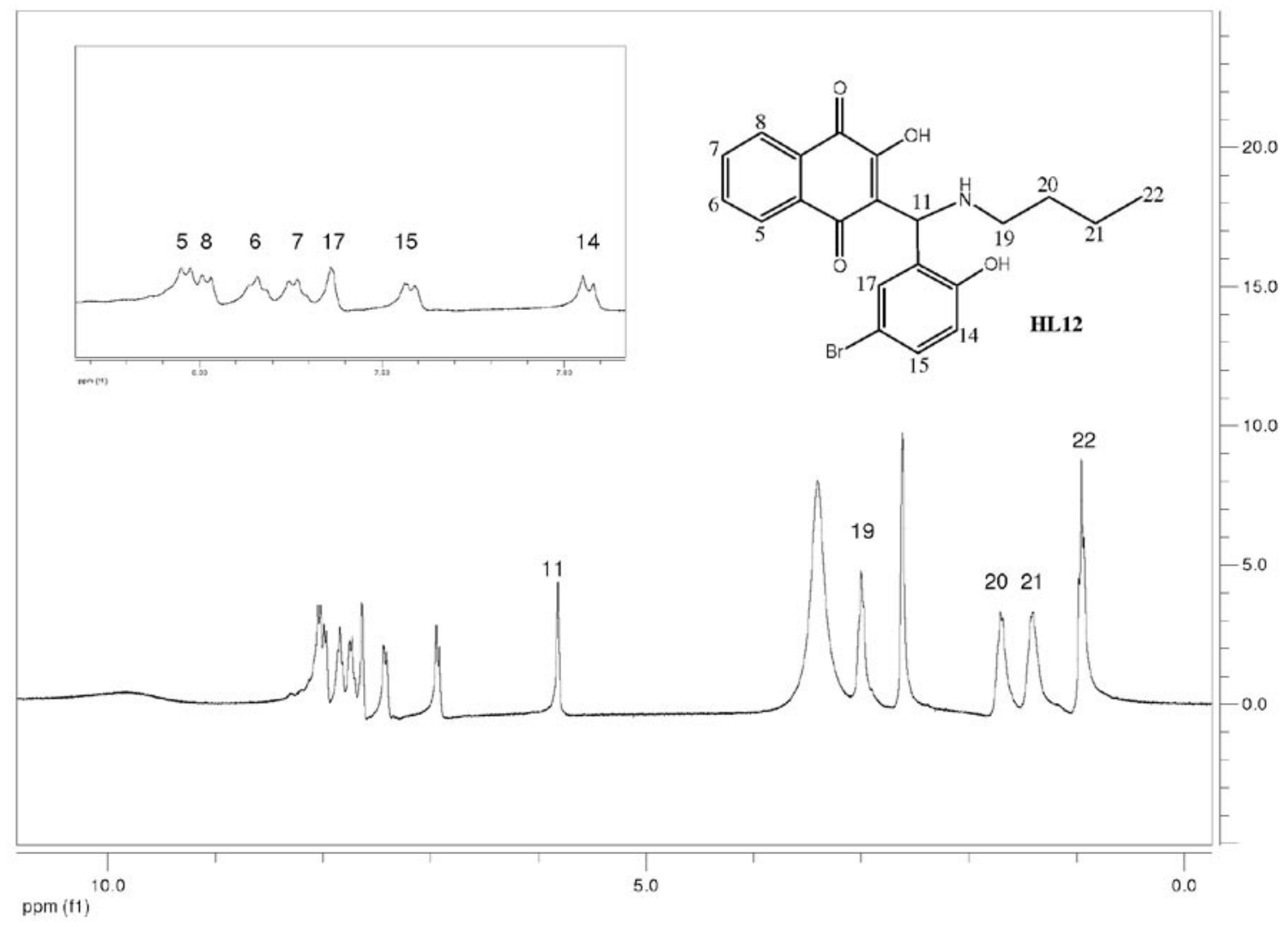

Figure S12. 'H NMR spectrum of HL12.

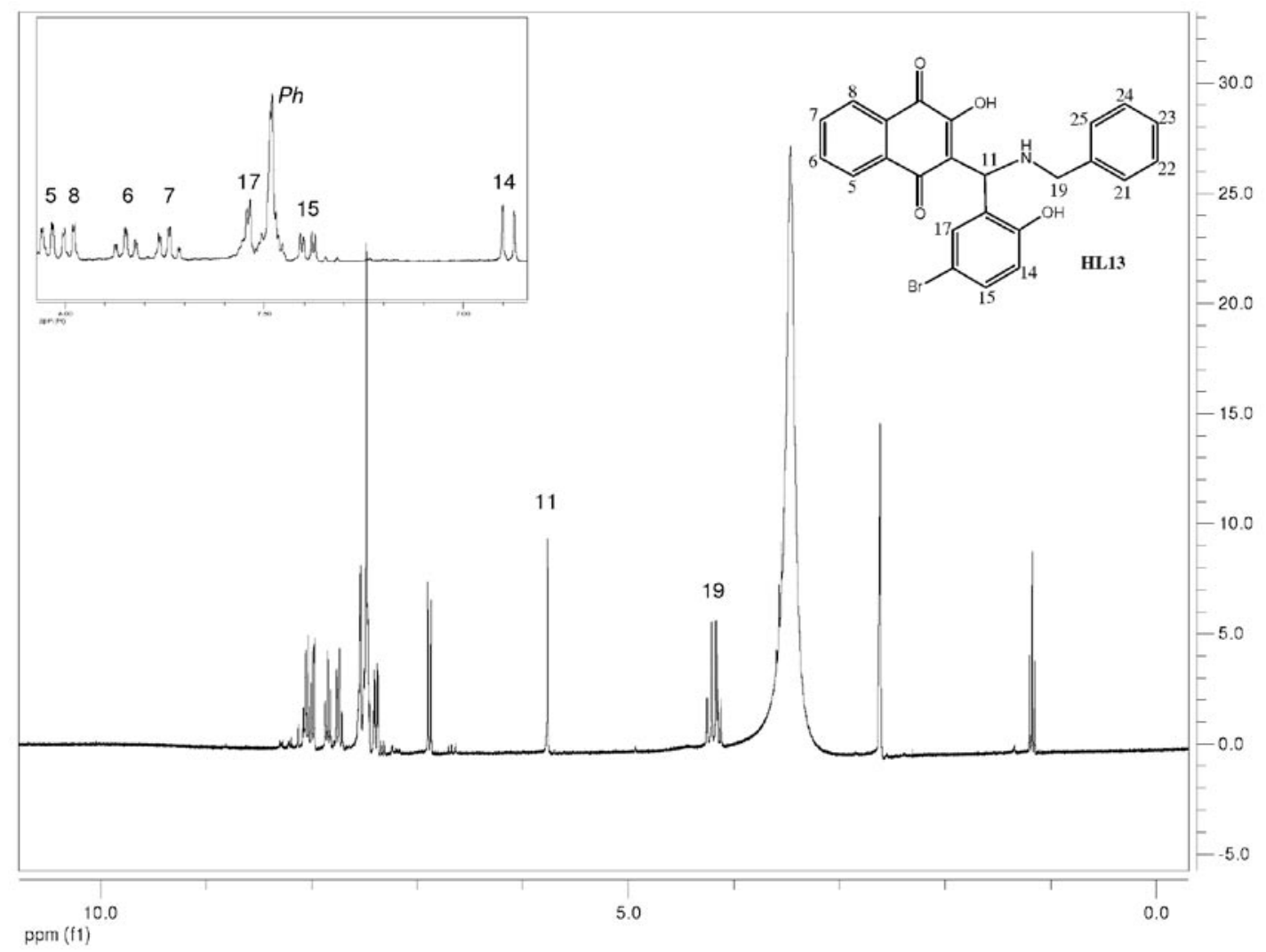

Figure S13. 'H NMR spectrum of HL13. 


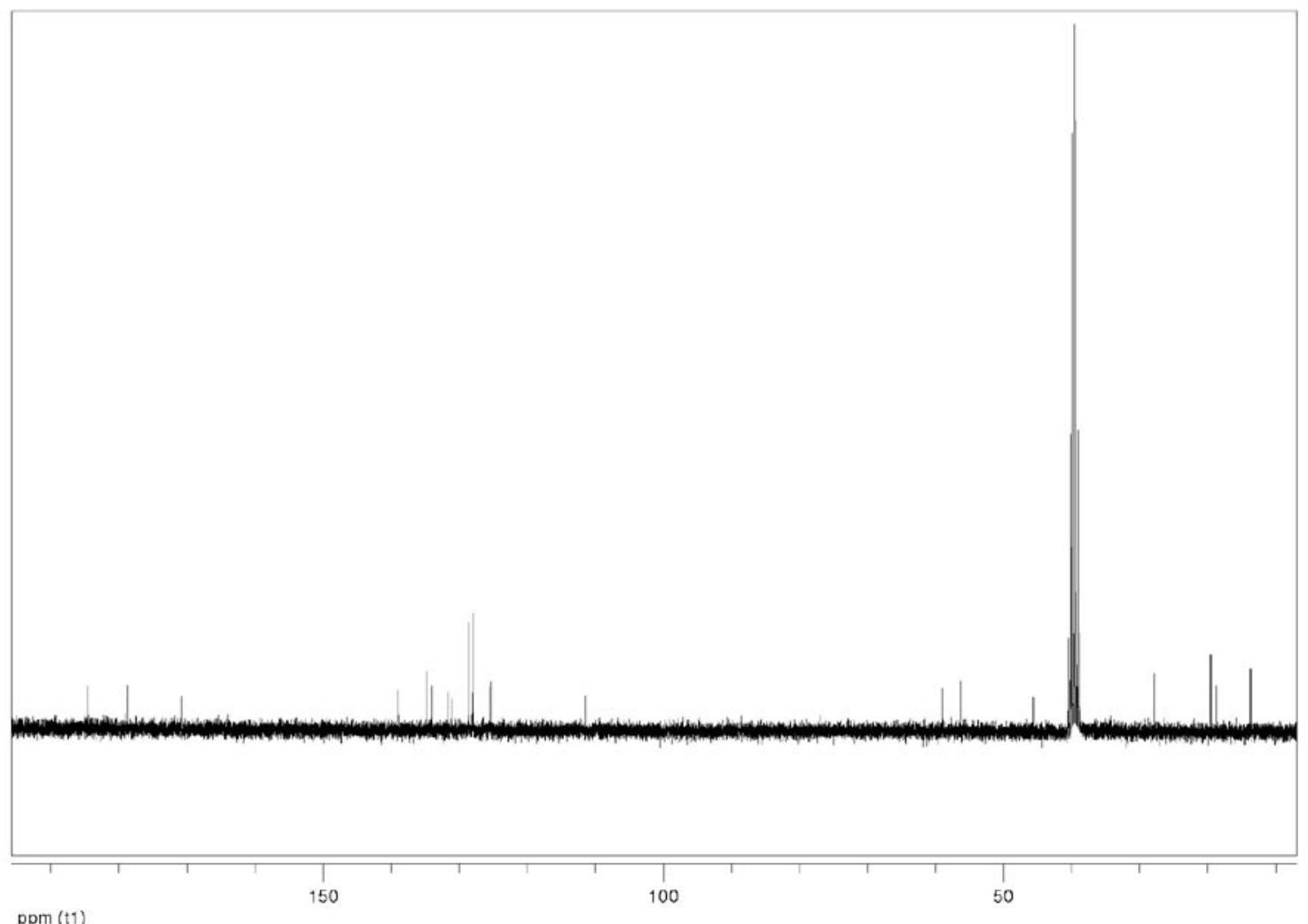

$\mathrm{ppm}($ (11)

Figure S14. ${ }^{13} \mathrm{C}$ NMR spectrum of HL1.

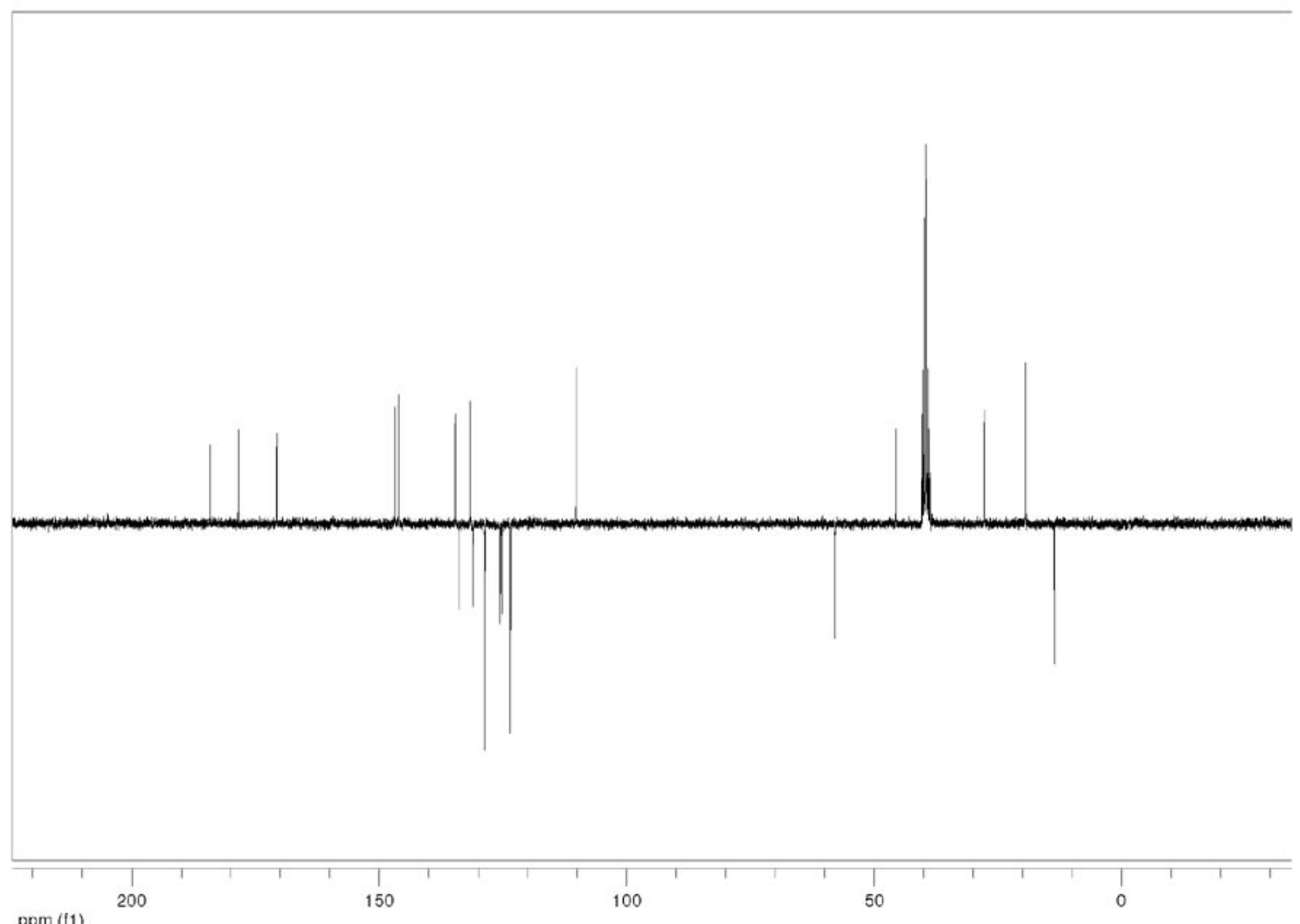

ppm (f1)

Figure S15. ${ }^{13} \mathrm{C}$ NMR spectrum of HL2. 


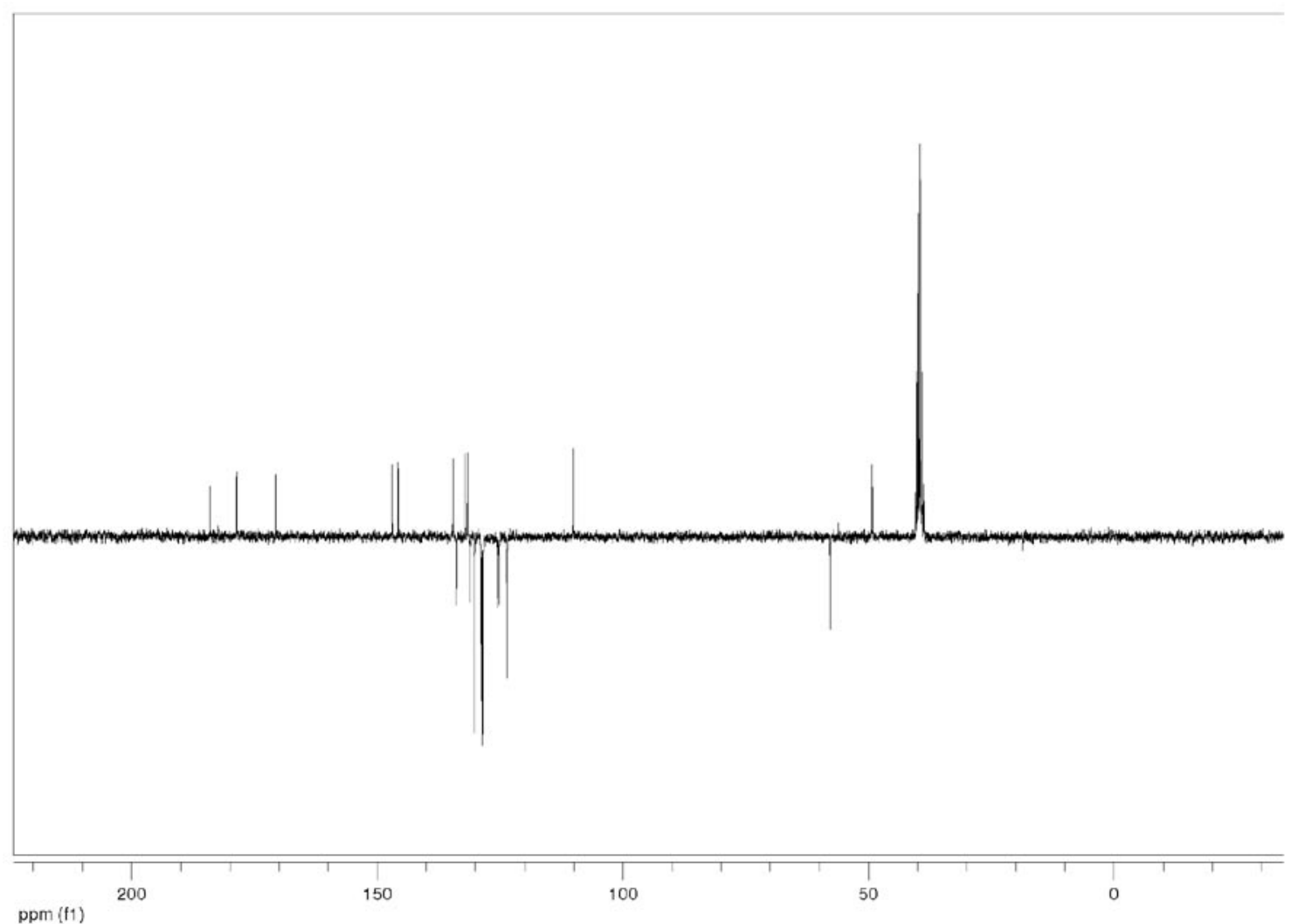

Figure S16. ${ }^{13} \mathrm{C}$ NMR spectrum of HL3.

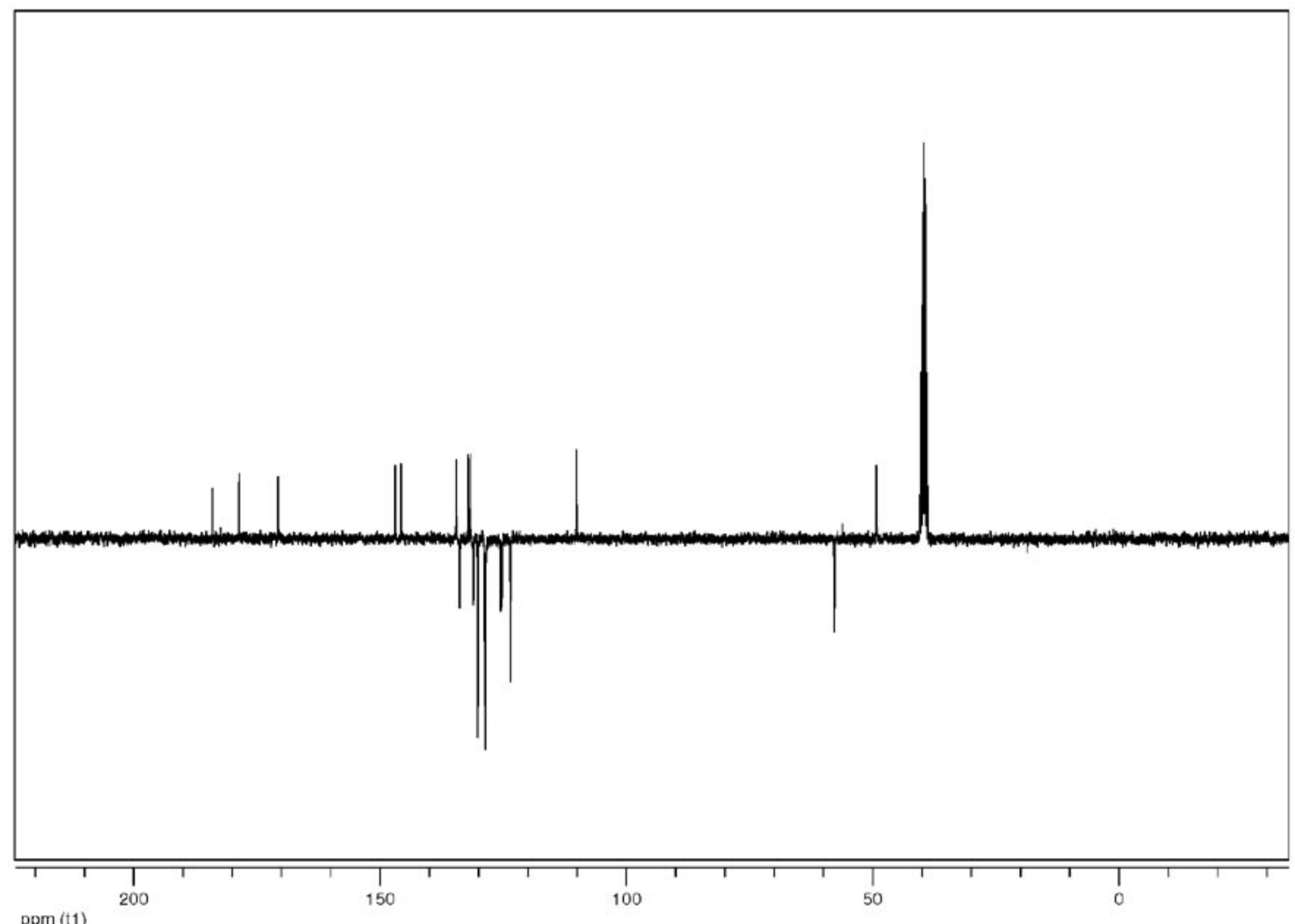

Figure S17. ${ }^{13} \mathrm{C}$ NMR spectrum of HL4. 


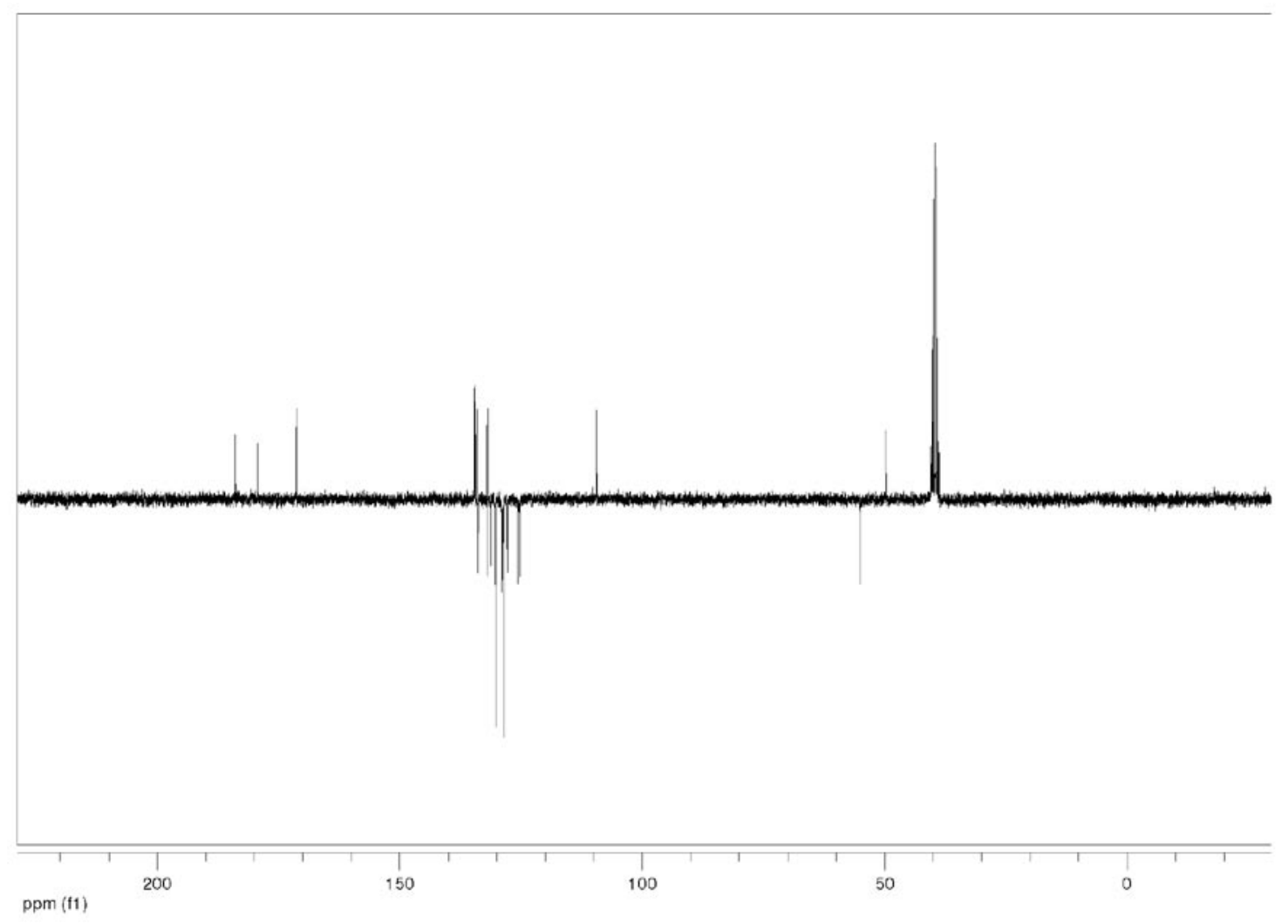

Figure S18. ${ }^{13} \mathrm{C}$ NMR spectrum of HL5.

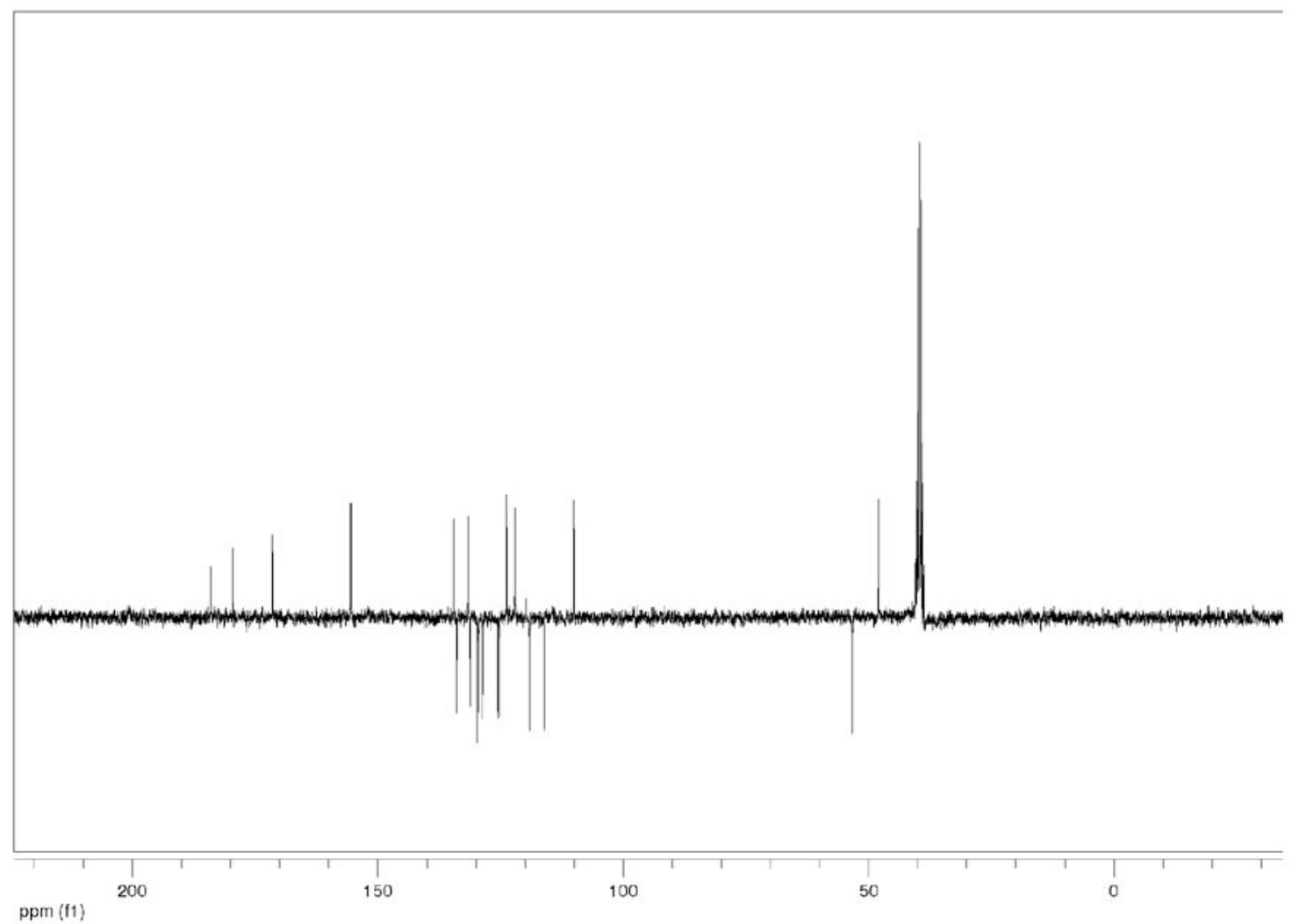

Figure S19. ${ }^{13} \mathrm{C}$ NMR spectrum of HL6. 


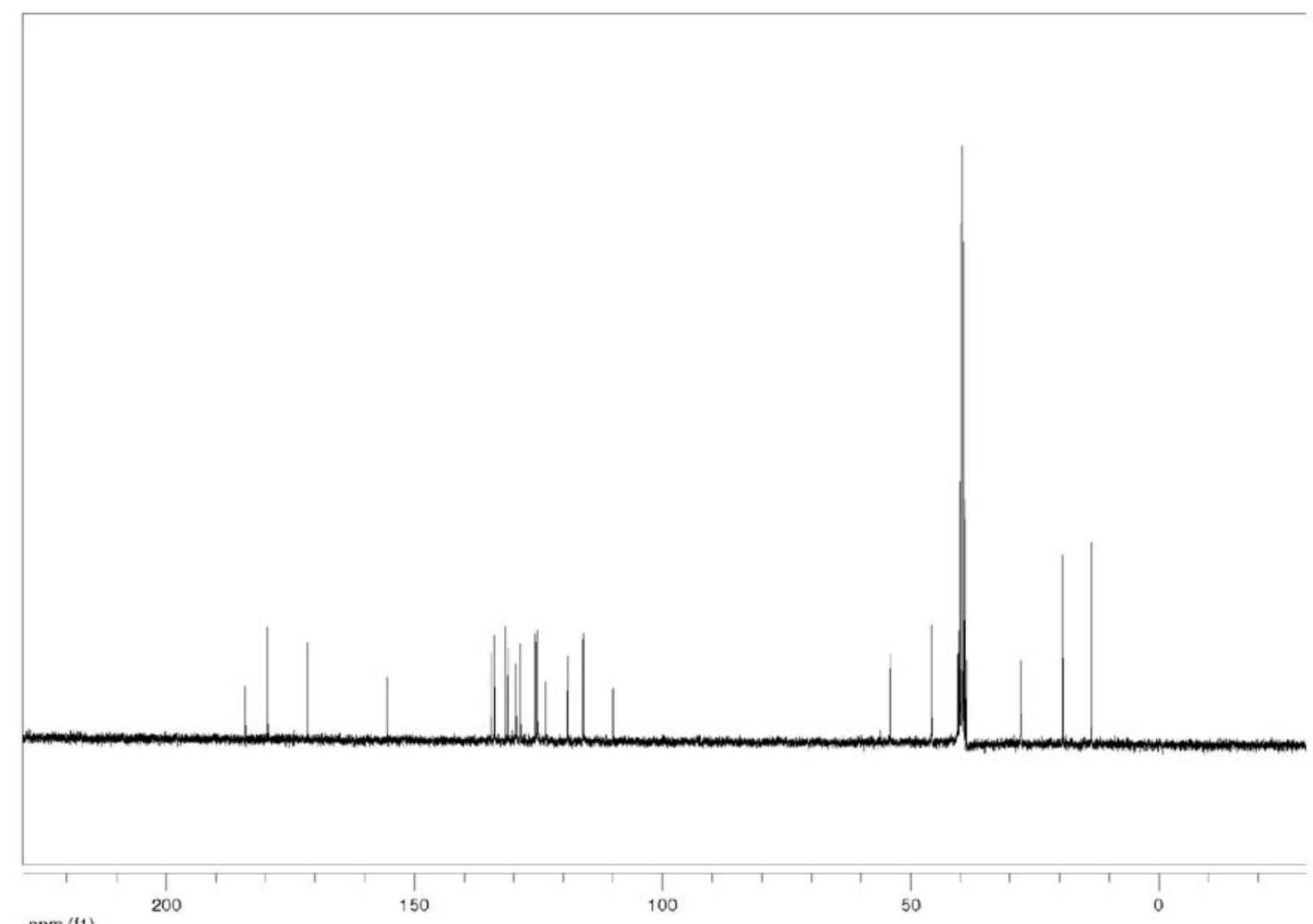

Figure S20. ${ }^{13} \mathrm{C}$ NMR spectrum of HL7.

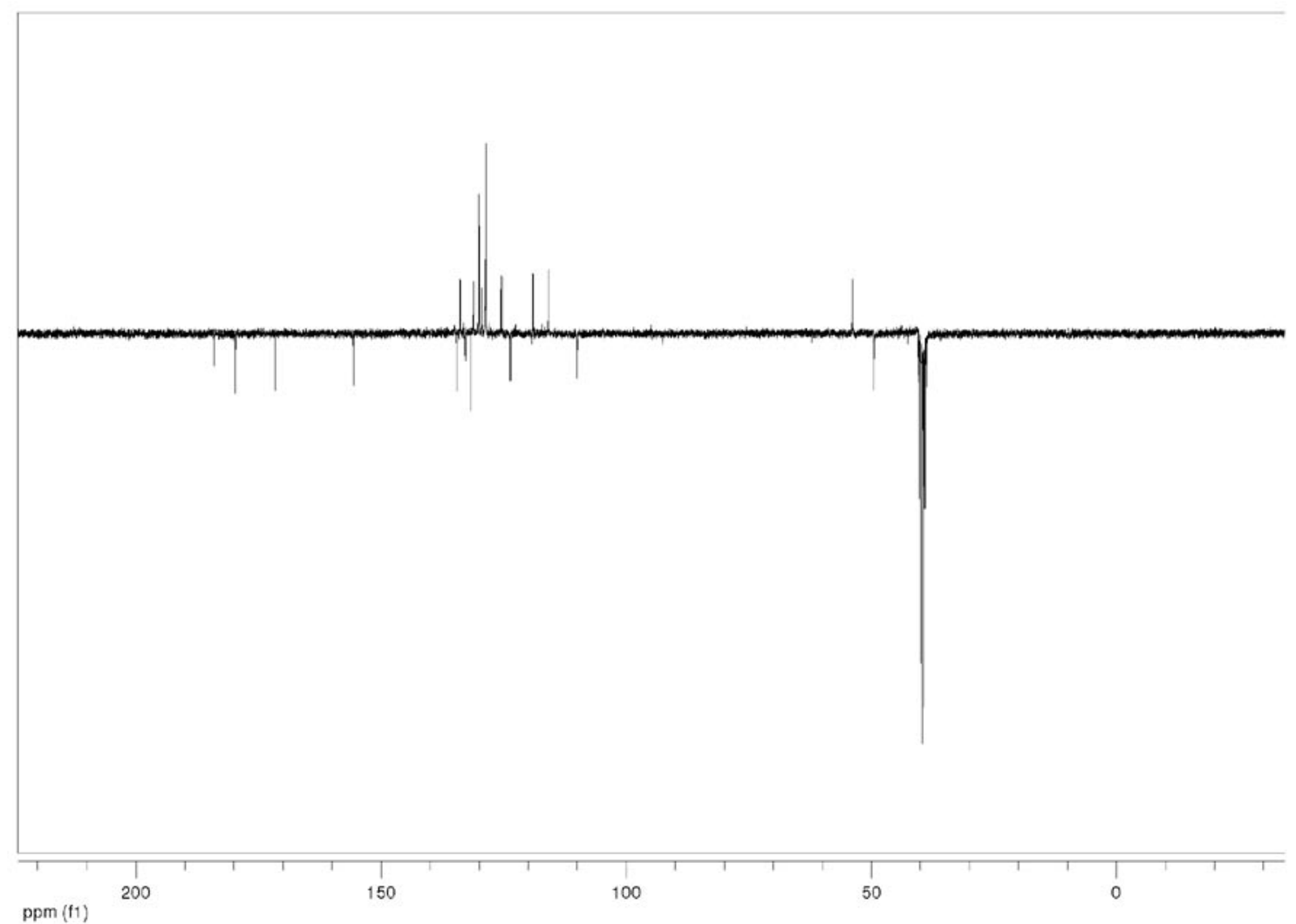

Figure S21. ${ }^{13} \mathrm{C}$ NMR spectrum of HL8. 


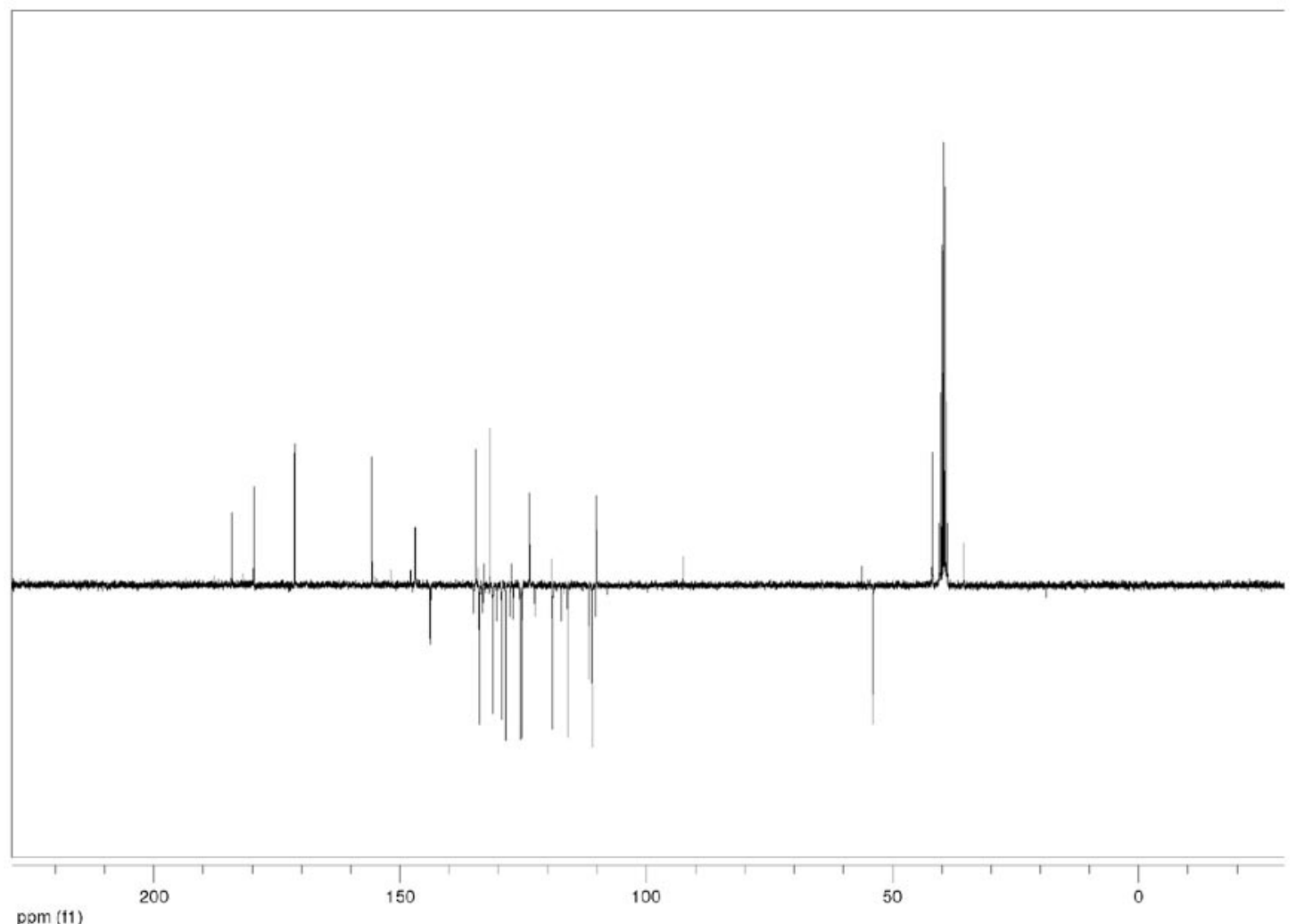

Figure S22. ${ }^{13} \mathrm{C}$ NMR spectrum of HL9.

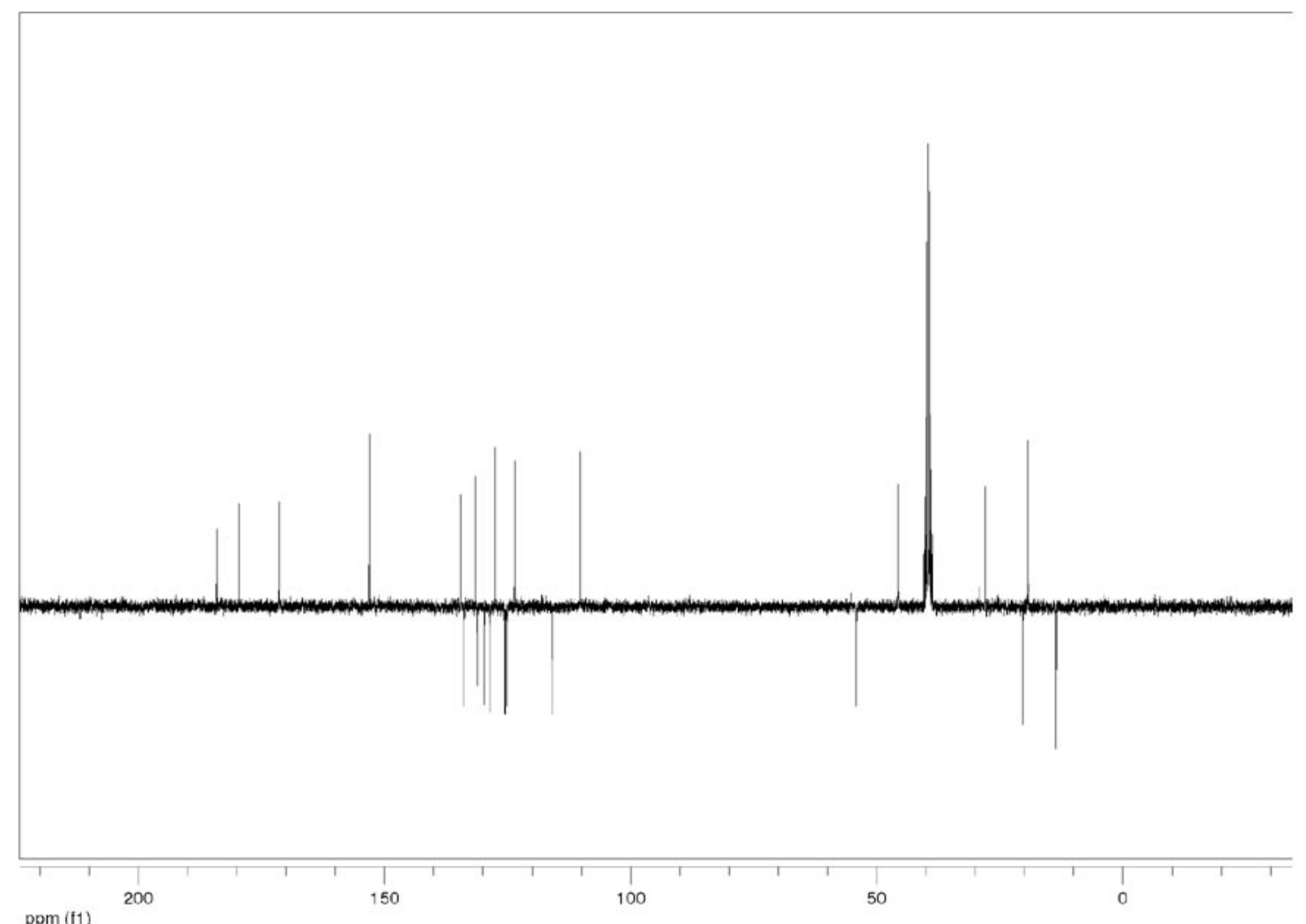

Figure S23. ${ }^{13} \mathrm{C}$ NMR spectrum of HL10. 


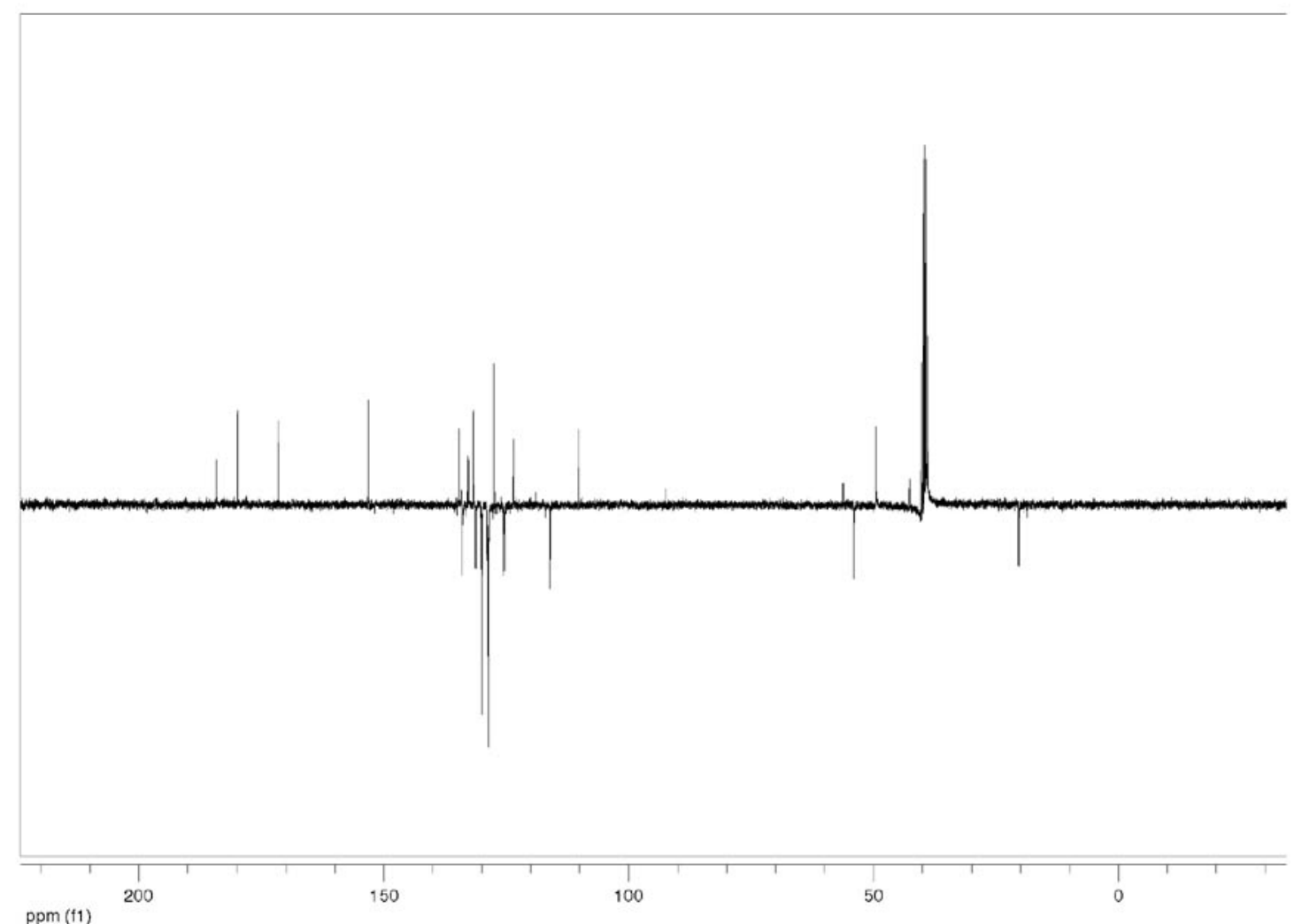

Figure S24. ${ }^{13} \mathrm{C}$ NMR spectrum of HL11.

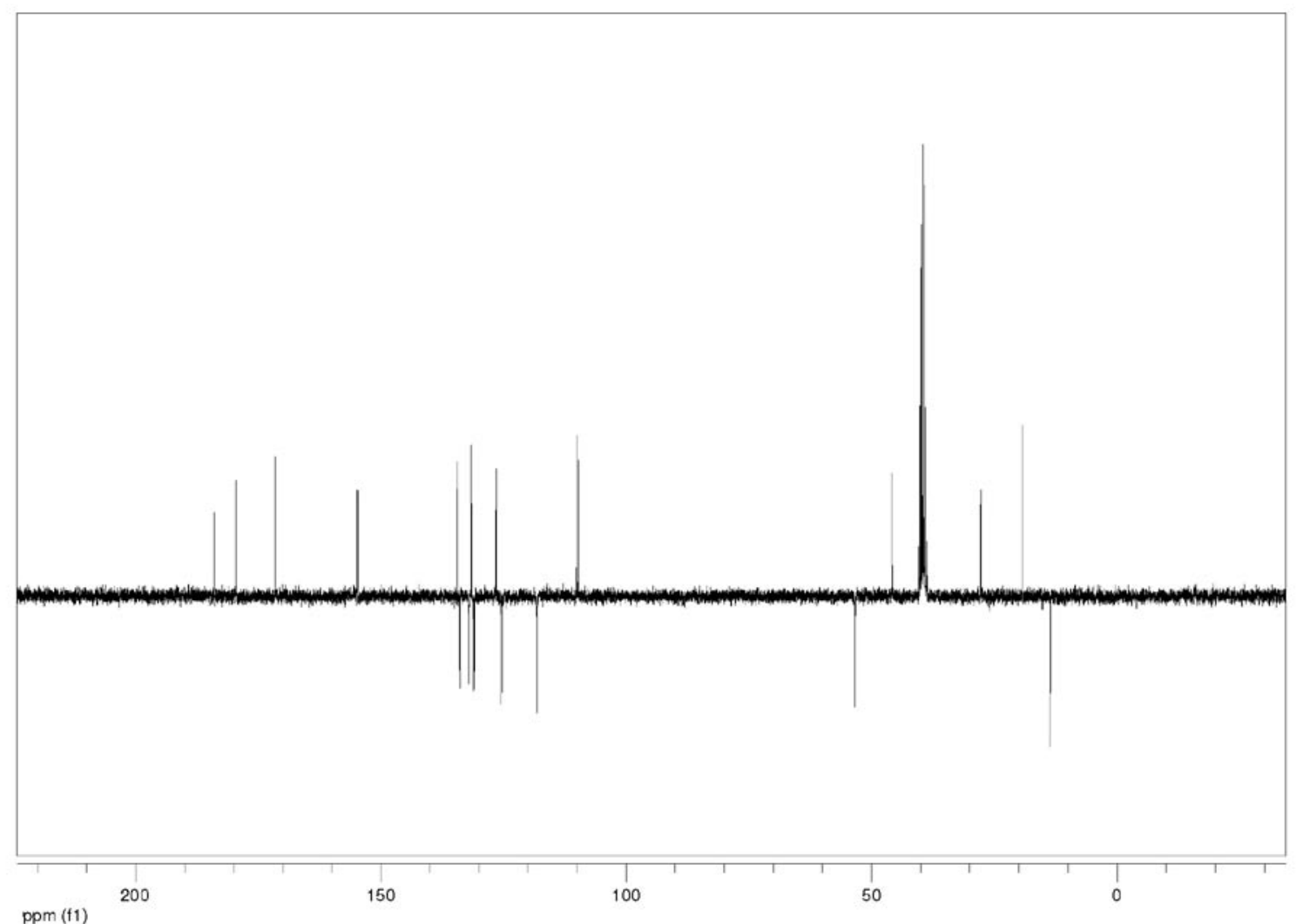

Figure S25. ${ }^{13} \mathrm{C}$ NMR spectrum of HL12. 


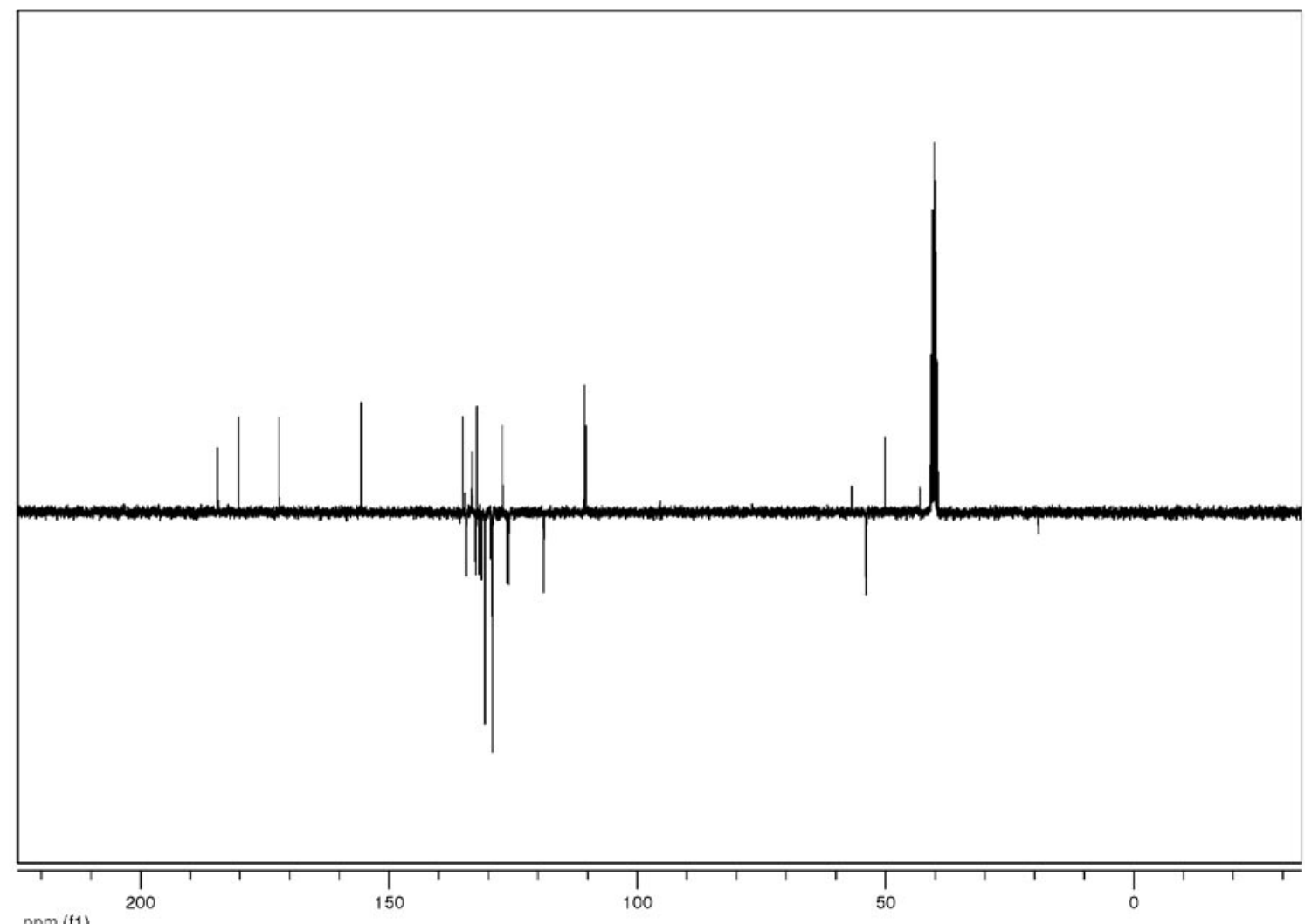

Figure S26. ${ }^{13} \mathrm{C}$ NMR spectrum of HL1. 